\author{
Universidade de São Paulo \\ Instituto de Física
}

\title{
Ricochetes não-singulares em teorias de gravidade modificadas
}

\author{
Ivan Yasuda
}

Orientador: Prof. Dr. Luís Raul Weber Abramo

Tese de doutorado apresentada ao Instituto de Física para a obtenção do título de Doutor em Ciências.

Banca Examinadora

Prof. Dr. João Carlos Alves Barata (Presidente-IFUSP)

Prof. Dr. Victor de Oliveira Rivelles (IFUSP)

Prof. Dr. Marcelo José Rebouças (CBPF)

Prof. Dr. Jailson Souza de Alcaniz (ON)

Prof. Dr. Júlio César Fabris (UFES)

São Paulo 
FICHA CATALOGRÁFICA

Preparada pelo Serviço de Biblioteca e Informação do Instituto de Física da Universidade de São Paulo

\begin{tabular}{|l|}
\hline Yasuda, Ivan \\
\hline Ricochetes não-singulares em teorias de gravidade \\
modificadas. - São Paulo, 2010 \\
Tese (Doutorado) - Universidade de São Paulo. \\
Instituto de Física. \\
Orientador: Prof. Dr. Luís Raul Weber Abramo \\
\hline Área de Concentração: Física \\
Unitermos: 1. Física; 2. Física Teórica; \\
3. Relatividade; Teoria de Campos. \\
USP/IF/SBI-044/2010
\end{tabular}




\section{Resumo}

Investigamos soluções de ricochete em uma teoria de gravidade modificada proposta por Brandenberger, Mukhanov e Sornborger. Mostramos, em particular, que um universo em contração, espacialmente plano e com fluidos usuais de matéria (poeira e radiação), conecta-se regularmente à fase de expansão acelerada antes de se unir ao período posterior de expansão desacelerada. A fase final emerge como uma expansão de de Sitter. No âmbito da teoria da relatividade geral, é conhecido o fato de que soluções de ricochete são possíveis apenas em universos abertos. Esta propriedade pode ser entendida em termos do tempo característico do ricochete, cuja duração, nesse contexto, possui um valor mínimo. No modelo que apresentamos, por outro lado, os ricochetes também ocorrem em universos planos, o que significa que a duração desta transição é arbitrária. Embora o modelo que investigamos seja de quarta ordem nas derivadas da métrica (sendo assim sujeito à instabilidade das perturbações), a dinâmica desta classe de soluções pode ser extendida a modelos motivados pela teoria de cordas capazes de acomodar ricochetes em universos planos. 


\begin{abstract}
We investigate bouncing solutions in the framework of a nonsingular gravity model of Brandenberger, Mukhanov and Sornborger. We show that a spatially flat universe filled with ordinary matter undergoing a phase of contraction reaches a stage of minimal expansion factor before bouncing in a regular way to reach the expanding phase. The expansion can be connected to the usual radiation- and matter-dominated epochs before reaching a final expanding de Sitter phase. In general relativity, a bounce can only take place provided that the spatial sections are positively curved, a fact that has been shown to translate into a constraint on the characteristic duration of the bounce. In our model, on the other hand, a bounce can occur also in the absence of spatial curvature, which means that the time scale for the bounce can be made arbitrarily short or long. Although the model we investigate is fourth order in the derivatives of the metric (and therefore unstable vis-à-vis the perturbations), this generic bounce dynamics should extend to string-motivated nonsingular models which can accomodate a spatially flat bounce.
\end{abstract}




\section{Agradecimentos}

Ao Prof. Dr. Luís Raul Weber Abramo pela orientação e ao Prof. Dr. Patrick Peter pela supervisão do meu estágio de doutoramento realizado no "Institut d'Astrophysique de Paris". A ambos, agradeço pelas anotações críticas feitas durante a elaboração da presente tese.

Ao Conselho Nacional de Desenvolvimento Científico e Tecnológico (CNPq) e à Coordenação de Aperfeiçoamento de Pessoal de Nível Superior (CAPES) pelo suporte financeiro.

Ao Instituto de Física da Universidade de São Paulo. Em particular, ao Departamento de Física Matemática, e ao "Institut d'Astrophysique de Paris", que propiciaram a estrutura técnica necessária para o desenvolvimento desta pesquisa.

Aos meus colegas e amigos do grupo, Rafael Ribeiro Brandão, Ronaldo Carlotto Batista e Vinicius de Souza Fernandes, pelas discussões sobre física. Agradeço particularmente ao Thiago dos Santos Pereira pelas críticas sobre este trabalho.

Aos meus amigos João Luís Meloni Assirati, Mário César Baldiotti, Suiane Fernandes, Rodrigo Fresneda e Milton Alexandre da Silva Júnior, pela generosidade e companheirismo.

Aos meus irmãos, Alexandre e Camila, pelo exemplo de dedicação e seriedade. Aos meus pais, Noboru e Maria, pela educação e pelo apoio e incentivo irrestritos. 
À Pascale e

À Sophie 


\section{Sumário}

1 Introdução $\quad 3$

2 Modelo cosmológico padrão $\quad 7$

2.1 Espaço-tempo de FRW . . . . . . . . . . . . . . 7

2.2 Dinâmica da expansão . . . . . . . . . . . . . . 14

3 Inflação $\quad 25$

3.1 Dinâmica inflacionária . . . . . . . . . . . . . 26

3.1 .1 Inflação caótica . . . . . . . . . . . . . 28

3.2 Perturbações de densidade . . . . . . . . . . . . . 30

3.3 Invariância do espectro primordial . . . . . . . . . . . . . 34

3.4 Quantização de perturbações de densidade . . . . . . . . . . 37

3.5 Comentários . . . . . . . . . . . . . . . 39

4 Singularidades $\quad 42$

4.1 Condições de energia . . . . . . . . . . . . . . . . . 42

4.2 Equação de Raychaudhuri e teorema de singularidade . . . . . . 45

5 Ricochete $\quad \mathbf{4 8}$

5.1 Solução dos problemas do modelo de FRW . . . . . . . . . 50

5.2 Ricochete clássico . . . . . . . . . . . . . . 51

5.2 .1 Matriz de transferência . . . . . . . . . . . 51

5.2 .2 Equações de movimento . . . . . . . . . . . 53

5.2 .3 Descrição do ricochete . . . . . . . . . . . . 55

5.2 .4 Modificação do espectro de flutuações . . . . . . . . . . 57

5.3 Cosmologia quântica . . . . . . . . . . . . . 58

5.4 Modelos pré-big bang . . . . . . . . . . . . . . 62

5.5 Comentários . . . . . . . . . . . . . . . 64 
6 Gravidade generalizada $\quad 68$

6.1 Teorias $f(R) \ldots \ldots \ldots \ldots$. . . . . . . . . . . . . . . . . 69

6.1 .1 Instabilidade de Ostrogradski . . . . . . . . . . . 71

6.1 .2 Ausência de fantasmas . . . . . . . . . . . . . . 73

6.1.3 Vínculos importantes . . . . . . . . . . . . . 75

6.1.4 Solução de ricochete . . . . . . . . . . . 79

6.1 .5 Perturbações de densidade . . . . . . . . . . . . . 82

6.2 Outras teorias a derivadas mais altas . . . . . . . . 87

6.2.1 Um modelo não-singular . . . . . . . . . . . . . 89

6.2.2 Ricochete .................. 95

6.2.2.1 Dinâmica das equações de campo . . . . . . 95

6.2.2.2 Curvatura espacial . . . . . . . . . . . 97

6.2.2.3 Gravidade vinculada com fluido de matéria . . 99

6.2.2.4 Escala de tempo típica do ricochete . . . . . . 104

6.2.3 Comentários ... . . . . . . . . . . 106

$\begin{array}{lll}7 & \text { Conclusões e perspectivas } & 108\end{array}$

$\begin{array}{ll}\text { Referências Bibliográficas } & 121\end{array}$ 


\section{Capítulo 1}

\section{Introdução}

As observações realizadas pelo satélite WMAP [1] ${ }^{1}$ indicam fortemente a ocorrência de um período inflacionário $[2,3,4]$ no universo primordial. A inflação não apenas oferece uma explicação para os problemas da planura e do horizonte intrínsecos ao modelo cosmológico padrão, como também implementam um mecanismo causal pelo qual perturbações da métrica são amplificadas a escalas maiores que o horizonte de Hubble com um espectro de potência aproximadamente invariante de escala. Entretanto, apesar de seus sucessos, a teoria inflacionária não aborda o problema da singularidade inicial. Apesar da condição de energia fraca ser violada em um cenário inflacionário típico, Borde, Guth e Vilenkin [5] demonstraram que espaços-tempos inflacionários são, de fato, geodesicamente incompletos na direção passada $[6,7]$.

Como uma alternativa à teoria inflacionária, modelos de ricochetes cosmológicos [8, 9, 10, 11, 12, 13], inspirados pela teoria de cordas, têm sido propostos. Exemplos são os cenários de "pré-big bang" 2 [14, 15] e modelos "ekpiróticos" [16, 17]. Assim como a inflação, ricochetes cosmológicos resolvem tanto o problema da planura - se a fase de contração for suficientemente mais longa do que a fase de expansão - como o problema do horizonte, ao permitir que regiões do passado correspondentes ao horizonte atual estivessem em contato causal [18].

Nos cenários de pré-big bang as equações de campo exibem uma simetria particular, que é a dualidade do fator de escala, cuja propriedade é mapear a fase de contração (no quadro de Einstein) - ou de pré-big bang - à fase de

\footnotetext{
1 "Wilkinson Microwave Anisotropies Probe"

${ }^{2}$ Devido ao seu uso extensivo e conhecido, vamos, a partir de agora, omitir as aspas entre a palavra inglesa "big bang", cuja tradução poderia ser "big bang".
} 
expansão do universo usual de Friedmann-Robertson-Walker - ou de pós-big bang. O problema agora está na transição entre as duas fases: os dois ramos não podem ser conectados um ao outro suavemente [19]. Posto de outra forma, enquanto a fase pré-big bang possui uma singularidade na direção futura, a fase pós-big bang emerge de uma.

A questão de como as flutuações da métrica poderiam ser afetadas por um ricochete também tem sido abordada, notavelmente no âmbito da relatividade geral. Uma abordagem clássica para essa questão de fato se justifica, ao menos no que se refere à sua descrição em eras bem anteriores, ou posteriores, ao ricochete, uma vez que, nesses regimes, é de se esperar que correções oriundas de uma física de altas energias possam ser ignoradas. No entanto, nas vizinhanças do ricochete, a aproximação clássica provavelmente perderá a sua validade, ao mesmo tempo em que correções sob a forma de termos efetivos podem se tornar importantes. Na ausência de uma teoria de altas energias capaz de descrever consistentemente o ricochete, o que se postula então é que, em analogia com outras transições curtas da cosmologia padrão - como o pré-aquecimento e a transição entre as eras dominada pela radiação e pela matéria - o tempo característico do ricochete é tal que o espectro das flutuações que por ele evoluem preservará seu caráter aproximadamente invariante de escala. Portanto, qualquer que seja a teoria sobre a qual se baseia o ricochete, é importante que se investigue a arbitrariedade do tempo que o caracteriza.

Realizar ricochetes em um contexto clássico não é uma tarefa trivial. Em um cenário com um único campo escalar em um universo aberto, o espectro no limite de grandes escalas exibe uma mistura entre os modos de Fourier [11]. Modelos sem curvatura espacial, mas com um campo de $k$-essência [20] como conteúdo material, também têm sido investigados [21]. Nesse caso, além do fato de observáveis físicos serem afetados pelo ricochete, tais cenários comportam fluidos fantasmas, que são fluidos cuja equação de estado é dada por $w \equiv$ $P / \rho \leq-1$, onde $P$ e $\rho$ são, respectivamente, a pressão e a densidade de energia do fluido ${ }^{3}$. Portanto, para que o ricochete possa se unir a uma era de expansão dominada por radiação, é necessária a introdução de um mecanismo

\footnotetext{
${ }^{3} \mathrm{Na}$ referência [22], Abramo e Pinto-Neto mostraram que fluidos fantasmas descritos por campos escalares generalizados (ou $k$-essência) estão sujeitos a três problemas fundamentais: (i) suas Hamiltonianas são ilimitadas inferiormente, o que significa que a teoria é instável, (ii) se a velocidade do som $c_{S}$ for tal que $c_{S}^{2} \geq 0$, então não há maneira da equação de estado cruzar o ponto $w=-1$ e (iii) a equação de estado e a velocidade do som são ilimitadas inferior e superiormente, respectivamente.
} 
de decaimento similar ao pré-aquecimento.

Neste trabalho [13], ao invés de investigarmos regularizações de cenários pré-big bang ou modelos clássicos de ricochete com algum conteúdo particular de matéria, estudaremos modelos cosmológicos baseados em uma teoria de gravidade a derivadas mais altas proposta em [23, 24]. Neste modelo, a ação gravitacional efetiva é construída de tal modo que todos os invariantes de curvatura são limitados, propriedade esta garantida pela introdução de multiplicadores de Lagrange e respectivos potenciais. Os limites apropriados são assim estabelecidos, isto é, em regime de alta curvatura, o vínculo sobre os invariantes garante que as soluções serão bem comportadas, e, em regime de curvatura baixa, recuperamos o limite einsteiniano.

Em um trabalho subseqüente [25], motivados principalmente pela resolução do problema da singularidade em cenários de pré-big bang, Brandenberger, Easther e Maia estudaram uma cosmologia dilatônica não-singular no contexto da teoria apresentada em [23, 24]. Aqui, estamos interessados em uma solução alternativa: vamos considerar um universo homogêneo e isotrópico com um fluido hidrodinâmico de poeira e radiação. A escolha dos invariantes de curvatura vai ditar a dinâmica do fator de escala, que se desenvolve a partir de um ricochete regular até o universo em expansão de Friedmann, se aproximando na fase final de uma era de expansão de de Sitter que poderia simular a época presente de aceleração do universo. Também investigaremos o problema da duração do ricochete, e mostraremos que, em oposição ao tratamento usual [11], ela é completamente arbitrária.

Extensões da teoria da relatividade geral envolvendo potências mais altas dos invariantes de curvatura são, de fato, bem motivadas, uma vez que cálculos de divergência de um "loop" (laço) em gravidade quântica [26, 27] geram termos na ação porporcionais a $R^{2}, R_{\mu \nu}^{2}$ e $R_{\mu \nu \rho \sigma}^{2}$. Mas conforme mostrou Stelle em 1977 [28], embora ações dessa forma possam definir uma teoria renormalizável, elas são problemáticas no que se refere à presença de campos fantasmas, que são graus de liberdade que se propagam com energia cinética negativa [29, 30]. Em conseqüência disto, a teoria torna-se altamente instável, no sentido de que o estado de vácuo (vazio) pode decair em uma coleção de estados com energias positivas e negativas. No nível clássico, o que se espera é que a instabilidade se manifeste sobre perturbações gravitacionais crescentes cujos modos carregam tanto energia positiva como energia negativa. Este fenômeno é conhecido na literatura como instabilidade de Ostrogradsky [31, 32], e está relacionado com 
o fato de que a Hamiltoniana, por causa da presença de termos de derivada de ordem maior que um na Lagrangeana, é ilimitada inferiormente.

Neste trabalho [13] vamos considerar um caso especial de uma teoria quadrática, uma teoria na qual os termos de derivada mais alta aparecem linearmente. Conseqüentemente, as equações para o espaço-tempo de fundo serão de segunda ordem. Este fato, entretanto, não basta para provar a estabilidade, visto que as equações para o setor perturbativo vão ser de ordem mais alta. A ação que consideramos, no entanto, decorre, por hipótese, de uma expansão nos invariantes de curvatura que, uma vez incorporada na teoria exata (por exemplo, na gravidade quântica), deveria ser bem comportada e, portanto, livre de instabilidades.

O texto está organizado da seguinte maneira:

O capítulo 1 contém uma revisão de aspectos relevantes do modelo cosmológico padrão. Nos três capítulos seguintes, sobre inflação, singularidades e ricochetes, capítulos 2, 3 4, respectivamente, discutimos as motivações e maneiras diferentes de se explicar os problemas clássicos do modelo cosmológico padrão. Esta discussão nos leva naturalmente ao capítulo 5, que discute modelos cosmológicos em teorias de gravidade generalizadas.

A notação adotada será esclarecida ao longo do texto. 


\section{Capítulo 2}

\section{Modelo cosmológico padrão}

Nós, habitantes da Via Láctea, observamos um universo que possui uma distribuição de radiação cósmica de fundo isotrópica de uma parte em $10^{5}$ [1]. Isto significa que, ao medirmos a temperatura de seu espectro em um intervalo angular de minutos de arco até as maiores escalas angulares $\left(180^{\circ}\right)$, as flutuações de temperatura $\Delta T / T$ são da ordem de $10^{-5}$.

A priori, não há razão para acreditarmos que observadores de outros pontos do universo comóveis com o fluido cósmico observariam um universo distinto do nosso. Assim, além de possuir uma distribuição isotrópica de radiação cósmica de fundo, podemos supor que o nosso universo é também homogêneo.

Homogeneidade e isotropia do espaço ${ }^{1}$ constituem as hipóteses fundamentais do Princípio Cosmológico que, juntamente com a teoria da relatividade geral, forma um dos pilares do modelo cosmológico padrão. Neste capítulo, vamos recapitular alguns de seus aspectos mais elementares.

\subsection{Espaço-tempo de FRW}

Com o intuito de definirmos a métrica de Friedmann-Robertson-Walker (FRW), é importante introduzirmos os conceitos de homogeneidade e isotropia mencionados nos parágrafos anteriores. Vamos iniciar esta seção lembrando de algumas propriedades importantes sobre vetores de Killing ${ }^{2}$.

A primeira delas diz respeito à relação entre campos de Killing e o ten-

\footnotetext{
${ }^{1}$ Se o espaço-tempo, e não apenas o espaço, fosse homogêneo e isotrópico, o universo seria estático, fato este que, como comentaremos adiante, vai de encontro com as observações de um universo em expansão.

${ }^{2}$ Ver, por exemplo, Capítulo 13 da referência [33].
} 
sor de curvatura de Riemann. Usando a fórmula de comutação de derivadas covariantes, dada por

$$
\xi_{\mu ; \rho \sigma}-\xi_{\mu ; \sigma \rho}=R_{\mu \rho \sigma}^{\lambda} \xi_{\lambda}
$$

e a regra cíclica

$$
R_{\sigma \rho \mu}^{\lambda}+R_{\mu \sigma \rho}^{\lambda}+R_{\rho \mu \sigma}^{\lambda}=0
$$

é possível mostrar [33] que os campos de Killing obedecem à equação

$$
\xi_{\mu ; \rho \sigma}=R_{\sigma \rho \mu}^{\lambda} \xi_{\lambda}
$$

Uma conclusão importante decorre da equação (2.3): dados os valores iniciais de $\xi_{\mu}(x)$ e $\xi_{\mu ; \nu}(x)$ no ponto $X$, podemos determinar $\xi_{\mu ; \nu \sigma}(X)$ pela fórmula (2.3). Da mesma forma, por derivações sucessivas da equação (2.3), obtemos as derivadas de ordem superior a dois de $\xi_{\mu}(x)$ no ponto $X$, todas elas dadas como combinações lineares de $\xi_{\mu}(X)$ e de $\xi_{\mu ; \nu}(X)$. Vemos então que todo vetor de Killing $\xi_{\mu}(x)$ é univocamente determinado pelos valores iniciais $\xi_{\mu}(X)$ e $\xi_{\mu ; \nu}(X)$. Em geral, podemos então escrever, para qualquer vetor de Killing particular $\xi_{\mu}^{(n)}(x)$ de uma dada métrica, a seguinte expressão:

$$
\xi_{\mu}^{(n)}(x)=A_{\mu}^{\lambda}(x ; X) \xi_{\lambda}^{(n)}(X)+B_{\mu}^{\lambda \nu}(x ; X) \xi_{\lambda ; \nu}^{(n)}(X)
$$

onde $A_{\mu}^{\lambda}$ e $B_{\mu}^{\lambda \nu}$ são funções que dependem da métrica e de $X$, mas não dependem de $\xi_{\mu}^{(n)}(X)$ nem de $\xi_{\mu ; \nu}^{(n)}(X)$. Ou seja, os coeficientes $A_{\mu}^{\lambda}$ e $B_{\mu}^{\lambda \nu}$ são os mesmos para qualquer vetor $n$.

Um conjunto de vetores de Killing $\xi_{\mu}^{(n)}(x)$ é dito ser independente se eles não satisfizerem a relação linear

$$
\sum_{n} c_{n} \xi_{\mu}^{(n)}(x)=0
$$

com os coeficientes $c_{n}$ constantes. Da equação (2.4), segue então que, em $N$-dimensões, há, no máximo, $N(N+2) / 2$ vetores de Killing independentes. De fato, consideremos $M$ vetores $\xi_{\mu}^{(n)}(x), n=1, \ldots, M$. Para cada $n$, há $N$ quantidades $\xi_{\mu}^{(n)}(X)$ e $N(N-1) / 2$ quantidades independentes $\xi_{\mu ; \nu}^{(n)}(X)^{3}$. Isto é, as quantidades $\xi_{\mu}^{(n)}(X)$ e $\xi_{\mu ; \nu}^{(n)}(X)$ podem ser vistas como as componentes $\operatorname{dos} M$ vetores $\xi_{\mu}^{(n)}(x)$ em um espaço de $N+N(N-1) / 2=N(N+2) / 2$

\footnotetext{
${ }^{3} \mathrm{O}$ número $N(N-1) / 2$ segue da própria definição de vetores de Killing, que é $\xi_{\mu ; \nu}+$ $\xi_{\nu ; \mu}=0$.
} 
dimensões. Note que, se $M>N(N+2) / 2$ então os $M$ vetores $\xi_{\mu}^{(n)}(X)$ não podem ser independentes e, portanto, satisfazem as relações

$$
\sum_{n} c_{n} \xi_{\mu}^{(n)}(X)=\sum_{n} c_{n} \xi_{\mu ; \nu}^{(n)}(X)=0
$$

Mas se as relações acima são válidas, então os vetores $\xi_{\mu}^{(n)}(x)$ satisfazem a relação linear (2.5). Logo, se $M>N(N+2) / 2, \xi_{\mu}^{(n)}(x)$ não são independentes.

Podemos agora introduzir os conceitos de espaços métricos homogêneos e isotrópicos.

Definimos um espaço métrico como sendo homogêneo se todo ponto pode ser levado a qualquer outro ponto de sua vizinhança por uma isometria. Assim, um espaço homogêneo deve admitir a existência de vetores de Killing $\xi^{\mu}(x)$ que podem assumir qualquer valor em pontos diferentes do espaço.

Definimos um espaço métrico como sendo isotrópico em torno de um ponto $X$ se existirem transformações de isometria tais que $\xi_{\mu}(X)=0$ (ou seja, o ponto $X$ permanece fixo) com a matriz antisimétrica $\xi_{\mu ; \nu}(X)$ podendo assumir qualquer valor (ou seja, dois vetores quaisquer em $X$ são levados um ao outro por uma rotação).

Um espaço métrico que contenha o máximo número de vetores de Killing independentes em cada ponto é dito espaço maximalmente simétrico. Portanto, das definições acima, espaços homogêneos e isotrópicos são também espaços maximalmente simétricos.

Para o nosso propósito, que é o de construir um espaço total (espaçotempo) que não é maximalmente simétrico mas que pode ser decomposto em termos de um sub-espaço maximalmente simétrico, a importância dos conceitos acima reside no seguinte teorema [33]: sejam $N$ e $M$ as dimensões do espaço "total" e do subespaço maximalmente simétrico, respectivamente; sejam $v^{a}$ as coordenadas de $N-M$ e $u^{i}$ as coordenadas de $M$ (para o caso FRW, $N-M=1$ ("tempo") e $M=3$ ("espaço")). Então, é sempre possível escolher as $u$-coordenadas de tal modo que a métrica de todo o espaço seja dada por:

$$
d s^{2}=g_{a b} d v^{a} d v^{b}+f(v)\left[d \boldsymbol{u}^{2}+\frac{k(\boldsymbol{u} \cdot d \boldsymbol{u})^{2}}{1-k \boldsymbol{u}^{2}}\right]
$$

onde $k=-1,0,+1$ correspondem a, respectivamente, um espaço de curvatura (constante) negativa, nula ou positiva. 
A métrica de FRW é um exemplo de um espaço-tempo que não é maximalmente simétrico mas que pode ser decomposto em termos de um subespaço maximalmente simétrico. Nesse caso $g_{a b} d v^{a} d v^{b}=g(v) d v^{2}$ (porque $N-M=4-3=1$ ). Redefinindo convenientemente novas coordenadas $t, r, \theta, \varphi$ por

$$
\begin{aligned}
\int^{t}(-g(v))^{1 / 2} d v & \equiv t \\
u^{1} & \equiv r \sin \theta \cos \varphi \\
u^{2} & \equiv r \sin \theta \sin \varphi \\
u^{3} & \equiv r \cos \theta
\end{aligned}
$$

o teorema acima nos dá

$$
d s^{2}=-d t^{2}+a^{2}(t)\left[\frac{d r^{2}}{1-k r^{2}}+r^{2}\left(d \theta^{2}+\operatorname{sen}^{2} \theta d \varphi^{2}\right)\right],
$$

onde $a(t) \equiv \sqrt{f(v)}$. O elemento de linha (2.7) define a métrica de FRW.

Em coordenadas $t, r, \theta, \varphi$ definidas em (2.7), as curvas fundamentais $\boldsymbol{x}=$ constante são geodésicas, pois os símbolos de Christoffel $\Gamma_{00}^{i}=0$. Portanto, as coordenadas de (2.7) são comóveis, no sentido de que uma partícula em repouso neste sistema de coordenadas permanecerá em repouso. Note ainda que, como $g_{00}=-1$, o intervalo de tempo $d t$ medido por um relógio comóvel é também o intervalo de tempo próprio medido em seu referencial de repouso.

O papel da função $a(t)$ é o de definir uma escala de distância física entre dois objetos comóveis, como podemos verificar ao calcularmos a distância própria em relação à origem, em um tempo $t$, de um objeto com coordenada radial $r$ :

$$
d(r, t)=a(t) \int_{0}^{r} \frac{d r^{\prime}}{\sqrt{1-k r^{2}}}=a(t) \times\left\{\begin{array}{cll}
\operatorname{sen}^{-1} r & , k=+1 \\
\operatorname{senh}^{-1} r & , & k=-1 \\
r & , & k=0
\end{array}\right.
$$

Como $r$ é independente do tempo no sistema de coordenadas $t, r, \theta, \varphi$, a distância própria de um objeto comóvel aumenta ou diminui com o fator de escala $a(t)$. Portanto, se o universo está se expandindo (contraindo), a distância própria deve aumentar (diminuir). A confirmação observacional de um universo em expansão (contração) decorre diretamente da observação de deslocamentos 
de linhas espectrais de galáxias. Para entender este fenômeno, vamos considerar frentes de onda eletromagnética emitidas por uma fonte localizada em $r_{1} \mathrm{e}$ que se propagam radialmente na direção da origem. Lembrando que fótons se propagam ao longo de geodésicas nulas caracterizadas por $d s^{2}=0$, segue de (2.7) que um sinal luminoso emitido de $r_{1}$ em $t=t_{1}$ atingirá a origem em um tempo posterior $t=t_{0}$ dado por

$$
\int_{t_{1}}^{t_{0}} \frac{d t}{a(t)}=\int_{0}^{r_{1}} \frac{d r}{\sqrt{1-k r^{2}}} .
$$

Calculando a diferencial da equação (2.8), vemos que a relação entre o intervalo de tempo de duas emissões sucessivas, $\delta t_{1}$, e o intervalo de tempo entre a recepção desses sinais, $\delta t_{0}$, é dada por

$$
\frac{\delta t_{1}}{a\left(t_{1}\right)}=\frac{\delta t_{0}}{a\left(t_{0}\right)} .
$$

Em termos das freqüências de emissão, $\nu_{1}=1 / \delta t_{1}$, e de recepção, $\nu_{0}=1 / \delta t_{0}$, temos

$$
\nu_{0} / \nu_{1}=a\left(t_{1}\right) / a\left(t_{0}\right)
$$

Se o universo está se expandindo $\left(a\left(t_{1}\right)<a\left(t_{0}\right)\right)$, temos um deslocamento de linhas espectrais para o vermelho: a freqüência de recepção decresce por um fator de $a\left(t_{1}\right) / a\left(t_{0}\right)$, o que é equivalente a um acréscimo do comprimento de onda por um fator de $a\left(t_{0}\right) / a\left(t_{1}\right)$, convencionalmente definido como

$$
1+z \equiv \frac{a\left(t_{0}\right)}{a\left(t_{1}\right)}
$$

onde $z$ mede o deslocamento para o vermelho de linhas espectrais. De modo análogo, se o universo está se contraindo $\left(a\left(t_{1}\right)>a\left(t_{0}\right)\right)$, temos um deslocamento de linhas espectrais para o azul $(z<0)$.

Para fontes próximas, podemos expandir o fator de escala como

$$
a(t)=a\left(t_{0}\right)+\dot{a}\left(t_{0}\right)\left(t-t_{0}\right)+\frac{\ddot{a}\left(t_{0}\right)}{2}\left(t-t_{0}\right)^{2}+\ldots,
$$

onde $H_{0} \equiv \dot{a}\left(t_{0}\right) / a\left(t_{0}\right)$ é a chamada constante de Hubble, de tal modo que

$$
z=H_{0}\left(t_{0}-t_{1}\right)+\frac{1}{2}\left(q_{0}+2\right) H_{0}^{2}\left(t_{0}-t_{1}\right)^{2}+\ldots
$$


onde

$$
q_{0} \equiv-\frac{1}{H_{0}^{2}} \frac{\ddot{a}\left(t_{0}\right)}{a\left(t_{0}\right)}
$$

é chamado de parâmetro de desaceleração. Nessa aproximação para z pequeno, $t_{0}-t_{1}$ corresponde à distância própria $d$ (em unidades de $c=1$ ). Portanto, o deslocamento de linhas espectrais de galáxias, que pode ser para o vermelho $\left(H_{0}>0\right)$ ou para o azul $\left(H_{0}<0\right)$, cresce linearmente com a distância em primeira ordem de aproximação:

$$
z=H_{0} d+\ldots
$$

As primeiras medidas de $z$ de outras galáxias foram realizadas por volta de 1910 pelo astrônomo Vesto Melvin Slipher [34]. Em seus dados, constavam 41 galáxias, das quais 36 apresentavam $z$ positivo, $0<z \leqslant 0.006$, e apenas 5 apresentavam $z$ negativo, $-0.001 \leqslant z<0$, com $z=-0.001$ correspondendo à nebulosa de Andrômeda M31. Posteriormente, entre 1918 e 1925, C. Wirz e K. Lundmark [35, 36, 37, 38, 39, 40, 41] descobriram um certo número de nebulosas espirais. No entanto, em nenhum desses casos foi possível determinar uma dependência linear de $z$ com a distância, sobretudo devido ao fato de que, além do movimento devido à expansão ou contração do fator de escala, galáxias possuem também suas próprias velocidades peculiares devidas à presença de campos gravitacionais de corpos vizinhos. Uma observação efetiva da dependência linear só seria possível para valores de $z$ tais que $z \gg 10^{-3}$. De fato, no começo dos anos 30, E. Hubble [42] mediu deslocamentos de linhas espectrais da ordem de $z \simeq 0.02$ em galáxias do aglomerado de Coma, tornando clara a dependência linear. Medidas mais recentes da constante de Hubble $H_{0}$ realizadas pelo grupo "HST $H_{0}$ Key" [43] usando dados de supernovas do tipo Ia dão o seguinte valor para $H_{0}$ :

$$
H_{0}=71 \pm 6 \mathrm{~km} \mathrm{~s}^{-1} \mathrm{Mpc}^{-1}
$$

A hipótese de homogeneidade e isotropia do Princípio Cosmológico impõe que não somente a métrica, mas também que o tensor de momento e energia da matéria, $T_{\mu \nu}$, assim como qualquer outro campo presente no universo, sejam invariantes de forma. Do contrário, os fluxos e densidade de matéria não seriam os mesmos vistos por observadores (comóveis) situados em pontos diferentes do espaço. Levando-se em conta as leis de transformação de tensores 
em um espaço maximalmente simétrico, concluímos que a matéria do universo é necessariamente descrita como sendo a de um fluido perfeito, cujo tensor de momento e energia é dado por:

$$
T_{\mu \nu}=(\rho+P) U_{\mu} U_{\nu}+P g_{\mu \nu} .
$$

$P=P(t)$ e $\rho=\rho(t)$ são, respectivamente, a pressão e a densidade de energia do fluido, funções apenas do tempo devido à homogeneidade do espaço, e relacionadas pela equação de estado $w=\rho / P$. Para matéria quente (radiação), $w=1 / 3$, ao passo que, para fluidos não-relativísticos, $w=0 . \quad w=-1$ corresponde à energia de vácuo. $U_{\mu}$ é o quadri-vetor de velocidade definido como

$$
U^{t} \equiv 1, U^{i} \equiv 0, i=1,2,3
$$

indicando o fato de que o conteúdo de matéria está em repouso no sistema de coordenadas $r, \theta, \varphi$.

O tensor de momento e energia (2.13) obedece à lei de conservação

$$
T_{; \nu}^{\mu \nu}=0
$$

As componentes $\mu=i$ da equação acima são trivialmente satisfeitas, enquanto que da componente temporal resulta a seguinte equação:

$$
\frac{d \rho}{d t}=-\frac{3}{a} \frac{d a}{d t}(1+w) \rho
$$

Integrando a equação (2.15) acima obtemos a seguinte solução para $\rho=\rho(a)$ :

$$
\rho \propto a^{-3(1+w)}=\left\{\begin{array}{ccc}
\text { constante } & , w=-1 \\
a^{-3} & , w=0 \\
a^{-4} & , w=1 / 3
\end{array}\right.
$$

O comportamento da densidade de energia como função do tempo depende da dinâmica do fator de escala que, por sua vez, se propaga segundo as equações do campo gravitacional. Veremos na seção seguinte que as equações de campo da teoria da relatividade geral indicam que universos homogêneos e isotrópicos, nos quais o conteúdo de matéria é descrito como um fluido perfeito, necessariamente originaram-se de um ponto singular, também chamado de big bang, no qual o fator de escala se anula e a densidade de energia diverge. 


\subsection{Dinâmica da expansão}

Para determinarmos a evolução do fator de escala, necessitamos resolver as equações do campo gravitacional de Einstein na presença de um fluido perfeito cujo tensor de momento e energia é dado pela equação (2.13). A ação total da relatividade geral compreende um primeiro termo gravitacional dado pela ação de Einstein-Hilbert e um segundo termo correspondendo à ação da matéria. Ou seja,

$$
S=\frac{1}{16 \pi G} \int R \sqrt{-g} d^{4} x+S_{m}\left[g_{\mu \nu}, \psi\right] .
$$

Nessa expressão $R$ é o escalar de Ricci, definido como $R=g^{\mu \nu} R_{\mu \nu}$, onde o tensor de Ricci $R_{\mu \nu}=R_{\mu \lambda \nu}^{\lambda}=\Gamma_{\mu \nu, \lambda}^{\lambda}-\Gamma_{\mu \lambda, \nu}^{\lambda}+\Gamma_{\mu \nu}^{\lambda} \Gamma_{\lambda \rho}^{\rho}-\Gamma_{\mu \rho}^{\lambda} \Gamma_{\nu \lambda}^{\rho}$, e $S_{m}$ é a ação da matéria, que depende da métrica e dos campos materiais representados conjuntamente pela variável $\psi$.

Variando a ação (2.17) com respeito à métrica, obtemos assim as equações de Einstein:

$$
R_{\mu \nu}-\frac{1}{2} g_{\mu \nu} R=8 \pi G T_{\mu \nu}
$$

onde $T_{\mu \nu}=-\frac{2}{\sqrt{-g}} \frac{\delta S_{m}}{\delta g^{\mu \nu}}$. Note que, como o tensor de momento e energia tem divergência nula, o lado esquerdo das equações (2.18) satisfaz

$$
\left(R_{\nu}^{\mu}-\frac{1}{2} \delta_{\nu}^{\mu} R\right)_{; \mu}=0
$$

igualdade esta que é automaticamente satisfeita devido às identidades de Biancchi. Portanto, as equações de conservação (2.14) estão contidas nas equações de Einstein. Em outras palavras, as equações de campo determinam não somente as componentes da métrica, mas também a distribuição e os fluxos que geram o campo gravitacional.

As equações de Einstein (2.18) constituem um sistema de equações diferenciais parciais de segunda ordem, apesar do fato da Lagrangeana de EinsteinHilbert conter termos que dependem da derivada segunda da métrica. Esses termos, no entanto, são lineares, de tal modo que, utilizando o teorema de Gauss, podem ser transformados em uma integral de hipersuperfície, sobre a qual a variação da métrica é nula. Conseqüentemente, os termos contendo derivadas segunda da métrica também se anulam. Conforme veremos, o fato da Lagrangeana não conter termos que dependem de derivadas de ordem superior a um tem uma implicância direta sobre a estabilidade da teoria. Em teorias 
ditas instáveis, cujas Lagrangeanas dependem de termos de derivada de ordem maior que um, perturbações em torno de um certo fundo vão divergir através da criação de ondas gravitacionais de amplitudes crescentes contendo tanto modos de energia positiva - não massivos (gravitons) - como modos de energia negativa - massivos (ghosts). Este fenômeno, comumente conhecido pelo nome de instabilidade de Ostrogradsky [31, 32], está intimamente ligado ao fato de que a Hamiltoniana é ilimitada inferiormente.

Vamos agora utilizar as equações de Einstein para descrevermos a evolução do universo de FRW. Nesse caso particular, substituindo a métrica de FRW, equação (2.7), nas equações (2.18), obtemos

$$
\begin{aligned}
H^{2}+\frac{k}{a^{2}} & =\frac{8 \pi G \rho}{3} \\
\dot{H}+H^{2} & =-\frac{4 \pi G}{3}(\rho+3 P)
\end{aligned}
$$

onde $H \equiv \dot{a} / a$ é o parâmetro de Hubble.

Podemos traçar um panorama geral do comportamento do fator de escala apenas por inspeção das equações de Friedmann, equações (2.19) e (2.20). A primeira delas nos diz que, contanto que a densidade de energia seja sempre positiva, como é o caso para fluidos de matéria usuais, notavelmente poeira e radiação, um universo em expansão deixará de se expandir em um instante $t$ apenas se a curvatura espacial $k$ for igual a +1 , ou seja, se o universo for fechado. De modo análogo, se o universo estiver em uma fase de contração, somente voltará a se expandir também se $k=+1$. Note que, para que o fator de escala atinja um valor mínimo, devemos ter $H=0$ e $\dot{H}>0$. É o que ocorre em modelos de ricochetes cosmológicos, que discutiremos à frente. A segunda equação de Friedmann nos diz, por sua vez, que, contanto que $\rho+3 P \geq 0$, então $\ddot{a} / a \leq 0$, ou seja, em um tempo passado finito, que escolhemos como sendo em $t=0$, o fator de escala necessariamente se anula. Nesse ponto, pelas equações (2.16), a densidade de energia vai a infinito. Além disso, se $\ddot{a} / a \leq 0$ e $k=+1$, a fase de contração posterior à fase de expansão necessariamente terminará em uma singularidade. É o que chamamos de grande trituramento. Antes de discutirmos com mais detalhes o problema da singularidade em $t=0$, voltemos ao estudo sobre o universo observável de Friedmann.

Para qualquer valor da constante de Hubble $H_{0}$, é útil definirmos a densi- 
dade crítica de energia atual, $\rho_{0, \text { crítico }}$, como

$$
\rho_{0, \text { crítico }} \equiv \frac{3 H_{0}^{2}}{8 \pi G}
$$

Da primeira equação de Friedmann (2.19), vemos que a curvatura espacial $k$ será $+1,0,-1$ conforme a densidade de energia atual do universo for maior,

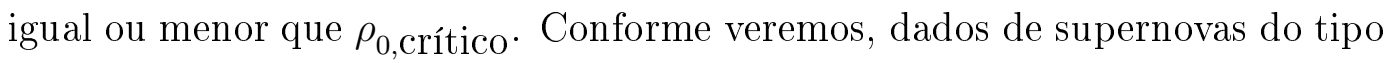
Ia e da radiação cósmica de fundo considerados em conjunto indicam que a densidade de energia atual é muito próxima da densidade crítica. Por outro lado, em tempos remotos, quando $a \rightarrow 0$, a densidade de energia era sobretudo dominada pela radiação, como vemos das equações (2.16), crescendo em direção ao passado como $a^{-4}$, de tal modo que a constante $k$ na primeira equação de Friedmann pode certamente ser desprezada face ao termo $\rho a^{2}$ presente no lado direito das equações. Portanto,

$$
\left(\frac{\dot{a}}{a}\right)^{2} \rightarrow \frac{8 \pi G \rho}{3} .
$$

Ou seja, em épocas primordiais, a densidade de energia era também muito próxima da densidade crítica. O fato curioso é que, após bilhões de anos de evolução, a densidade de energia total do universo permanece muito próxima da densidade crítica. Esse fato é conhecido como problema da planura. Uma boa explicação, e também a mais simples, é que o universo sempre foi plano e que a densidade crítica corresponde exatamente à densidade total de energia. Outras soluções são evidentemente possíveis, como, por exemplo, uma fase primordial de expansão acelerada ( $\ddot{a}>0$ ), ou inflação $[2,3,4]$, anterior à fase da radiação, dominada pela energia de vácuo $(w=-1)$, durante a qual o termo de curvatura $k / a^{2} H^{2} \rightarrow 0$ rapidamente; ou ainda um ricochete cosmológico, cuja fase de contração é suficientemente mais longa que a fase de expansão [18], conforme discutiremos à frente.

As medidas de pequenos $z$ 's de galáxias próximas como função da distância permitiram aos astrônomos determinarem a constante de Hubble $H_{0}$. Nesses casos, do fato de serem fontes próximas, para as quais $z$ não excede o valor 0.1 e para as quais a expansão para $z$, equação (2.12), ainda é válida, os efeitos da evolução cosmológica sobre as medidas de distância podem ser desprezados. Em se tratando de fontes mais longínquas, no entanto, para as quais $z>0.1$, a interpretação dos dados necessita de uma teoria dinâmica para a expansão. 
Tendo em vista este propósito, é útil reescrevermos a primeira equação de Friedmann (2.19) em termos dos chamados parâmetros de densidade, definidos em termos da densidade crítica de energia (2.21) como

$$
\Omega_{X} \equiv \frac{\rho_{0, X}}{\rho_{0, \text { crítico }}}
$$

onde $\rho_{0, X}$ se refere a qualquer um dos componentes da densidade de energia total atuais: $X=$ radiação $(R)$, matéria não-relativística $(M)$, vácuo $(\Lambda)$. Em termos de $\Omega_{X}$ temos então:

$$
\Omega_{\Lambda}+\Omega_{M}+\Omega_{R}+\Omega_{k}=1
$$

onde $\Omega_{k} \equiv-k / a_{0}^{2} H_{0}^{2}$. Uma quantidade de interesse é o parâmetro de desaceleração, definido em (2.11), que podemos expressar em termos dos parâmetros de densidade:

$$
q_{0}=\frac{1}{2}\left(\Omega_{M}-2 \Omega_{\Lambda}+2 \Omega_{R}\right) .
$$

O sinal negativo diante do parâmetro de densidade da energia de vácuo indica que essa componente tende a fazer com que a expansão seja acelerada, ao contrário do parâmetro de densidade da matéria (fria ou quente), cujo efeito é desacelerar a expansão.

Uma conseqüência importante das equações de Friedmann é que o universo deve ter evoluído de um estado quente e denso com a matéria e a radiação em equilíbrio termodinâmico. Portanto, é de se esperar que, naquela época, o espaço fosse preenchido por uma radiação de corpo negro [44, 45], cujo espectro é dado por

$$
n_{T}(\nu) d \nu=\frac{8 \pi \nu^{2} d \nu}{e^{\frac{h \nu}{k_{B} T}}-1},
$$

onde $n_{T}(\nu) d \nu$ é a densidade de número de fótons com freqüência entre $\nu$ e $\nu+d \nu$ e $T$ é a temperatura de equilíbrio. Devido à expansão do fator de escala, a temperatura diminuiu progressivamente, os fótons foram se diluindo e seus comprimentos de onda estirados. Logo, se $t_{L}$ é o instante de tempo do desacoplamento entre radiação e matéria, em um instante de tempo posterior $t$, temos

$$
n(\nu, t) d \nu=\left(\frac{a\left(t_{L}\right)}{a(t)}\right)^{3} n_{T\left(t_{L}\right)}\left(\nu \frac{a(t)}{a\left(t_{L}\right)}\right) d\left(\nu \frac{a(t)}{a\left(t_{L}\right)}\right) .
$$




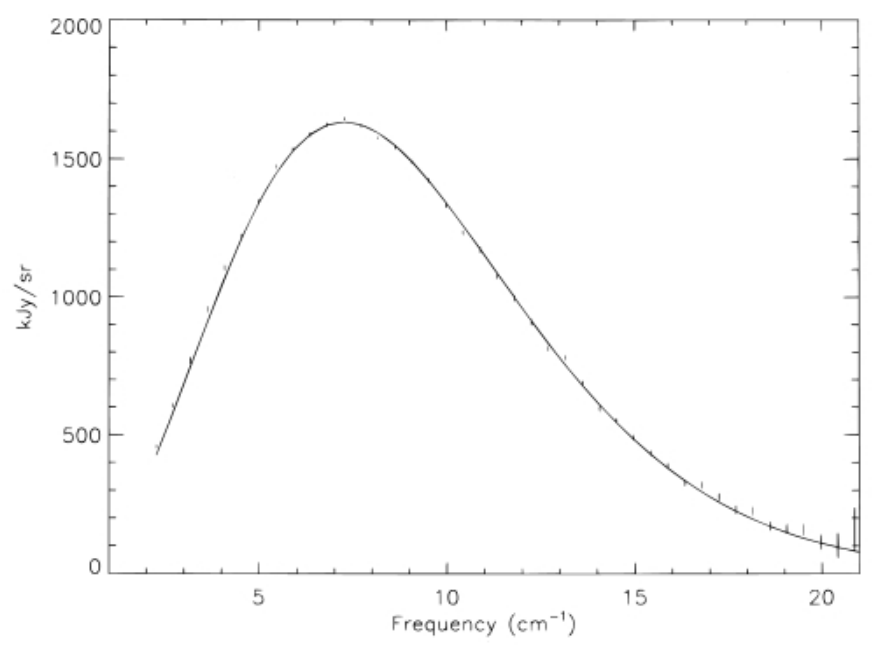

Figura 2.1: Comparação entre a intensidade da radiação cósmica de funda observada pelo radiômetro FIRAS e a radiação de corpo negro com temperatura $T=2.78 \mathrm{~K}$. Figura extraída de [46].

O primeiro fator do lado direito dá conta da diluição de fótons, e o argumento $\nu \frac{a(t)}{a\left(t_{L}\right)}$ do deslocamento para o vermelho da freqüência dos fótons. Substituindo (2.25) em (2.26), obtemos

$$
n(\nu, t) d \nu=\frac{8 \pi \nu^{2} d \nu}{e^{\frac{h \nu}{k_{B} T(t)}}-1}
$$

onde $T(t)=T\left(t_{L}\right) a(t) / a\left(t_{L}\right)$. Ou seja, a forma de espectro de corpo negro é preservada em instantes de tempo $t>t_{L}$, mas com uma temperatura menor por um fator de $a(t) / a\left(t_{L}\right)$.

Esta previsão do modelo cosmológico padrão indicando a existência de uma radiação cósmica de fundo com espectro de corpo negro foi verificada na década de 90 pelo radiômetro FIRAS do satélite COBE ("Cosmic Background Explorer Satellite") [46]. A comparação entre a intensidade da radiação observada com o espectro de corpo negro de temperatura $T_{0}=2,78 \mathrm{~K}$ é mostrada na figura (2.1).

Um teste de importância do modelo cosmológico padrão diz respeito à abundância de elementos leves [47, 48, 49, 50]. A síntese desses elementos $\left({ }^{2} \mathrm{H}\right.$, ${ }^{3} \mathrm{He},{ }^{4} \mathrm{He} \mathrm{e}{ }^{7} \mathrm{Li}$ ) remonta a uma época entre um a mil segundos depois do big bang, quando a temperatura do universo estava em torno de $10^{10} \mathrm{~K}$, portanto, a um período bem anterior ao desacoplamento, quando $t_{L} \sim 10^{5}$ anos e $T\left(t_{L}\right) \sim$ $10^{4} \mathrm{~K}$. Assim como neste último caso, em que o único parâmetro ajustável é a temperatura $T_{0}$, as previsões da nucleossíntese do modelo cosmológico padrão 


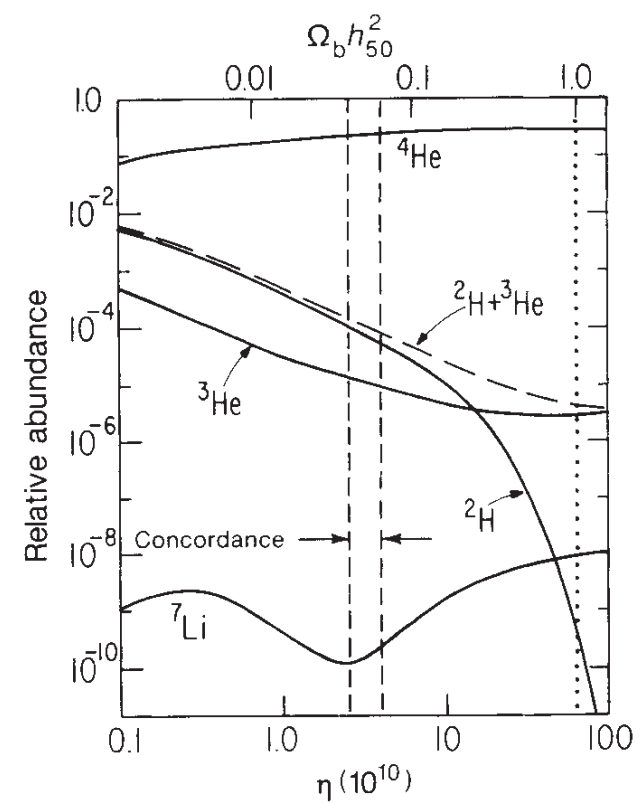

Figura 2.2: Previsões do modelo cosmológico padrão para as abundâncias relativas de elementos leves. $\Omega_{b}$ é o parâmetro de densidade de bárions, $\eta$ é a razão entre a densidade de bárions e a densidade de fótons e $h_{50}$ é a constante de Hubble em unidades de $50 \mathrm{Km} \mathrm{s}^{-1} \mathrm{Mpc}^{-1}$. A faixa vertical corresponde ao vínculo sobre $\Omega_{b}$. Figura baseada na referência [49] e extraída de [51].

também envolvem um único parâmetro livre, a saber, a razão $\eta \equiv n_{B} / n$, onde $n_{B}$ é a densidade de bárions e $n$ é a densidade de fótons (conhecida dos dados da radiação cósmica de fundo). A consistência entre essas previsões e as abundâncias observadas implicam um parâmetro de densidade de bárions $\Omega_{\text {bárions }} h_{50}^{-2}=0,05 \pm 0,01$ [49], corroborando assim a existência de uma componente escura, chamada de matéria escura, na densidade de matéria do universo.

Como mencionamos anteriormente, dados da radiação cósmica de fundo indicam que $-0,0178<k / a_{0}^{2} H_{0}^{2}<0,0063$ com $95 \%$ de confiança estatística [1]. Adicionalmente, dados de 42 supernovas do tipo Ia do grupo "Supernova Cosmology Project" [52,53] com $z$ entre 0,18 e 0,83 indicam, para uma cosmologia plana com $\Omega_{k}=0=\Omega_{R}$, de tal modo que $\Omega_{\Lambda}+\Omega_{M}=1$, que $\Omega_{M}=0,28 \pm 0,09$. Nesse caso, $\Omega_{\Lambda}=0,72$ e $q_{0}=-0,58$. Ou seja, os dados experimentais indicam fortemente que o universo está se expandindo aceleradamente. Em vista disso, como decorre da segunda equação de Friedmann, equação (2.20) (onde $\dot{H}+H^{2}=\ddot{a} / a$ ), uma fração majoritária do conteúdo energético do universo está sob uma forma tal que $\rho+3 P<0$, ou seja, sob a forma de um fluido com 

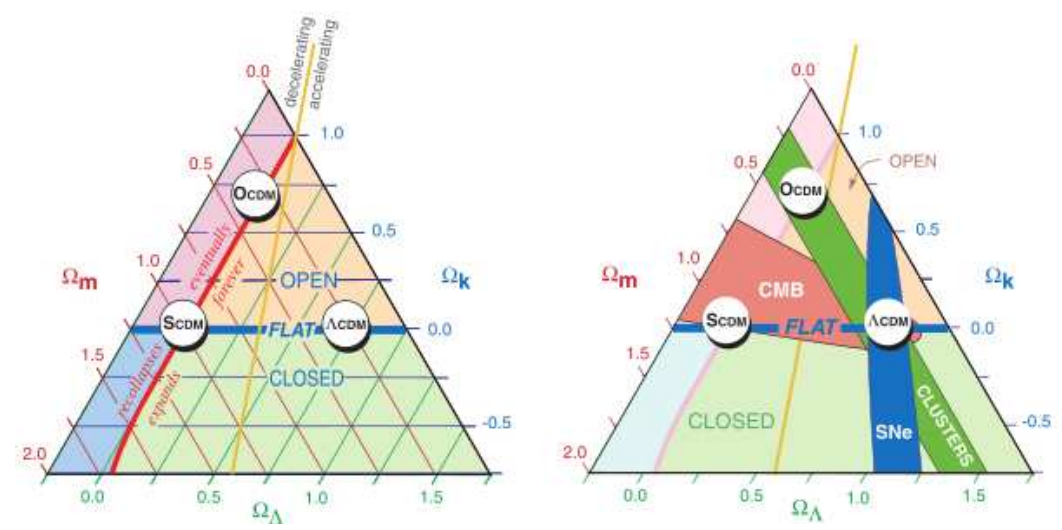

Figura 2.3: o triângulo cósmico, representando o conjunto de dados de aglomerado de galáxias (clusters), de supernovas ( $\mathrm{SNe}$ ) e da radiação cósmica de fundo (CMB). Cada ponto dentro do triângulo satisfaz $\Omega_{M}+\Omega_{\Lambda}+\Omega_{k}=1$. A linha avermelhada separa universos que se expandem para sempre ou que podem colapsar; a linha amarela separa universos em expansão acelerada daqueles em expansão desacelerada; por fim, pontos sobre a linha azul correspondem a universos planos. Há três modelos representados: SCDM $\left(\Omega_{M}=1\right)$, OCDM $\left(\Omega_{\Lambda}=0, \Omega_{M}=1 / 3, \Omega_{k}=2 / 3\right)$ e $\Lambda \operatorname{CDM}\left(\Omega_{\Lambda}=2 / 3, \Omega_{M}=1 / 3, \Omega_{k}=0\right)$. Esta figura foi extraída de [52].

pressão negativa. Este novo tipo de componente é comumente denominado de energia escura.

A combinação de dados oriundos da observação de aglomerados de galáxias ( $z$ pequeno), supernovas do tipo Ia ( $z$ médio) e da radiação cósmica de fundo ( $z$ grande) pode ser representada pelo triângulo cósmico [52], reproduzido na figura (2.3).

Embora as observações de um universo em expansão acelerada sejam consistentes com a existência de uma energia de vácuo constante, a sua natureza ainda permanece um verdadeiro enigma. Em particular, é profundamente misteriosa a razão pela qual a sua densidade é tão pequena [54]. Flutuações quânticas em campos conhecidos em escalas de energia de $300 \mathrm{GeV}$, que é a escala em que teorias correntes são suscetíveis de verificação, dão uma energia de vácuo da ordem de $10^{27} \mathrm{~g} / \mathrm{cm}^{3}$, ou seja, maior por 56 ordens de grandeza que a densidade de energia escura, que é da ordem de $\Omega_{\Lambda} \rho_{0, \text { crítico }} \simeq 10^{-29} \mathrm{~g} / \mathrm{cm}^{3}$. Esta diferença indica que deve haver outras contribuições desconhecidas para a energia de vácuo, vindas de flutuações de campos em altas energias ou das próprias equações de movimento, capazes de cancelar a contribuição que resulta no valor discrepante de $10^{27} \mathrm{~g} / \mathrm{cm}^{3}$. A razão para este cancelamento, que deve ser preciso por 56 casas decimais, por outras contribuições desconhecidas da energia de vácuo permanece um enorme mistério [54]. 
Um caminho possível na tentativa de se compreender a natureza da energia escura é através de teorias de gravidade modificada. Nesses casos, a expansão acelerada deixa de ser um fenômeno provocado por uma matéria desconhecida para ser descrita como um efeito puramente gravitacional. As teorias mais em voga nesse sentido são as chamadas teorias $f(R)$, onde $f(R)$ é uma função arbitrária do escalar de Ricci $R$ e que generaliza a ação de EinsteinHilbert. De uma forma geral, os modelos $f(R)$ propõem modificações no regime infra-vermelho, o que quer dizer no regime de baixas curvaturas escalares, correspondendo, portanto, a modificações em épocas recentes da história do universo.

Como veremos com um certo detalhe mais à frente, uma característica importante dos modelos $f(R)$ é que, apesar de serem a derivadas mais altas, eles podem ser estáveis [55, 31, 32]. De fato, ao escrevermos a teoria como uma teoria escalar-tensorial, verificamos que o grau de liberdade escalar é um "bom" campo, no sentido de não ser um fantasma ("ghost").

Modelos $f(R)$ consistentes com $(i)$ vínculos cosmológicos, ou seja, que são capazes de descrever corretamente todas as épocas do universo, e com (ii) vínculos de sistema solar, além, é claro, de obedecerem a condições de estabilidade, têm sido propostos [56, 57, 58, 59, 60, 61]. Um problema importante, entretanto, ainda está em aberto, a saber, como o grau de liberdade escalar, responsável nesta descrição pelo atual estado de expansão acelerada do universo, deve ter se comportado em épocas primordiais. O que se sabe é que, quando $t \rightarrow 0$, uma produção excessiva de partículas escalares, chamadas "escalarons" [57, 58], decorrente de oscilações do escalar de Ricci (oscilações cujas amplitudes divergem em $t=0)^{4}$, implica violações de condições de estabilidade, levando quantidades físicas a evoluírem através de uma singularidade ${ }^{5}$.

Após esta breve digressão sobre teorias $f(R)$, que estudaremos adiante com mais detalhes, voltemos ao estudo do modelo cosmológico padrão. Duas questões relevantes emergem naturalmente neste contexto: a primeira delas diz respeito à isotropia da radiação cósmica de fundo, e a segunda, conforme já comentamos anteriormente, diz respeito ao fato do universo ser praticamente

\footnotetext{
${ }^{4}$ Argumentos existem segundo os quais os modos oscilatórios com amplitudes divergentes são devidos ao fato de que modelos de energia escura em $f(R)$ têm uma singularidade no passado situada bem próxima do mínimo do potencial do campo escalar [62, 59].

${ }^{5}$ Esta singularidade corresponde ao ponto em que $d^{2} f / d R^{2}=0$, ponto no qual a massa do campo escalar vai a infinito $\left(M_{\phi} \simeq f^{\prime} / 3 f^{\prime \prime}\right)$. A condição de estabilidade impõe que $d^{2} f / d R^{2}>0$. Portanto, para que seja violada, $d^{2} f / d R^{2}$ deve necessariamente ir a zero em algum momento.
} 
plano.

\section{Problema do horizonte}

De fato, ao considerarmos a história térmica do universo, verificamos que fótons da radiação cósmica de fundo oriundos de regiões do céu separadas de uma distância angular maior ou igual a 1 grau não estavam em contato causal no passado, notavelmente na época do último espalhamento fóton-bárion, quando, devido ao resfriamento do universo, a radiação se desacoplou da matéria e passou a se propagar livremente. Vemos isto ao considerarmos um comprimento de onda físico $a \lambda$ tal que, em um dado instante de tempo, obedece à relação $a \lambda \lesssim H^{-1}$. Se $a \propto t^{n}$, onde $0<n<1$, então $a \lambda \propto t^{n}$ e $H^{-1} \propto t$. Portanto, em um futuro suficientemente distante, o comprimento de onda físico será bem menor que a escala de Hubble, o que significa que regiões causalmente conexas correspondem apenas a uma pequena fração do horizonte de Hubble.

Podemos também expressar esse fato em termos do horizonte de partículas, que define a distância percorrida por fótons desde a criação do universo (em $t=0)$, e que é definido por

$$
D_{H}(t)=a(t) d_{H}(t) \equiv a(t) \int_{0}^{t} \frac{d t^{\prime}}{a\left(t^{\prime}\right)} .
$$

$d_{H}(t)$ é chamado de horizonte de partículas comóvel. A razão entre $d_{H}\left(t_{\text {desac }}\right)$,

onde $t_{\text {desac }}$ é o instante de tempo do desacoplamento, e $d_{H}\left(t_{0}\right)$, onde $t_{0}$ é o instante de tempo presente, é então

$$
\frac{d_{H}\left(t_{\text {desac }}\right)}{d_{H}\left(t_{0}\right)} \approx\left(\frac{t_{\text {desac }}}{t}\right)^{1 / 3} \approx\left(\frac{10^{5}}{10^{10}}\right)^{1 / 3} \approx 10^{-2}
$$

Portanto, regiões causalmente conexas na época do desacoplamento eram muito menores que o horizonte de partículas atual.

\section{Problema da planura}

Vamos agora considerar o problema da planura. Observações de supernovas do tipo Ia $[52,53]$ são consistentes com um universo de curvatura espacial nula. Este fato implica um parâmetro de densidade total $\Omega(t) \equiv 8 \pi G \rho / 3 H^{2}$ cuja diferença da unidade é da ordem de $10^{-16}$ na época da nucleossíntese e da 
ordem de $10^{-64}$ na era de Planck [63]. Uma pequena perturbação em torno do fino ajuste desta condição inicial, do fato de $\Omega_{k}=-k / a^{2} H^{2}=0$ ser um ponto de instabilidade, levaria o universo a se colapsar ou a se expandir rapidamente antes que qualquer estrutura pudesse se formar. A instabilidade do ponto $\Omega_{k}=0$ pode ser vista ao derivarmos a primeira equação de Friedmann, equação (2.19), em relação ao tempo. Obtemos

$$
\frac{d}{d t}|\Omega-1|=-\frac{2 \ddot{a}}{\dot{a}^{3}}
$$

Se o universo se expande $(\dot{a}>0)$ desaceleradamente $(\ddot{a}<0),|\Omega-1|$ é uma função crescente do tempo, ou seja, $\Omega$ se distancia cada vez mais da unidade: o universo plano $(\Omega=1)$ é instável.

\section{Possíveis soluções}

Os problemas apresentados acima repousam no fato de que, no modelo cosmológico padrão, o universo está em expansão desacelerada. Vamos supor então que o universo se expandiu aceleradamente, ou seja, que ocorreu inflação. Nesse caso,

$$
\ddot{a}>0 \text {. }
$$

Da segunda equação de Friedmann, equação (2.20), isto implica

$$
\rho+3 P<0
$$

o que, conforme veremos, corresponde a uma violação da condição de energia forte. A condição (2.31) significa essencialmente que $\dot{a}=a H$ cresce durante a inflação, ao passo que o raio de Hubble comóvel $(a H)^{-1}$ decresce nesse período.

Problema do horizonte: O fator de escala pode ser escrito como $a \propto t^{n}$, onde agora $n>1$. Como $H^{-1} \propto t$, os comprimentos de onda físicos são estirados para além do raio de Hubble. Note que, nesse caso, a integral (2.28) diverge quando $a(0) \rightarrow 0$. Uma vez terminada a inflação, o raio de Hubble começa a crescer mais rápido que o comprimento de onda. Portanto, para que o problema do horizonte seja resolvido, é necessário que a distância comóvel percorrida por fótons durante a inflação seja muito maior que essa mesma distância percorrida após o desacoplamento. Um cálculo detalhado mostra, de fato, que o período de expansão acelerada deve durar, no mínimo, 70 tempos 
de Hubble [64].

Problema da planura: A inflação $(\ddot{a}>0$ e $\dot{a}>0)$ resolve o problema da planura ao impor que $|\Omega-1|$ decresce rapidamente. Depois dessa fase, o universo começa a se expandir desaceleradamente, de modo que $|\Omega-1|$ cresce com o tempo. No entanto, desde que a inflação seja suficientemente longa para levar $\Omega$ bem próximo da unidade, $|\Omega-1|$ permanecerá desprezível mesmo na era presente. 


\section{Capítulo 3}

\section{Inflação}

Como discutimos até o momento, o modelo cosmológico padrão fornece uma descrição consistente e bem testada da história do universo que vem desde a era da síntese dos elementos leves, a mais ou menos $10^{-2}$ segundos depois do big bang, até a era presente, a 15 bilhões de anos depois.

Não obstante seus sucessos, o modelo homogêneo e isotrópico de FRW não fornece uma resposta aos problemas levantados no final do último capítulo. Com o propósito de preencher esta lacuna, Guth propôs, em 1981 [2], a teoria inflacionária. Neste cenário, chamado de inflação velha, a inflação é gerada por um campo escalar, o inflaton, inicialmente preso em um falso vácuo onde ele age essencialmente como uma constante cosmológica, causando desta forma a expansão acelerada do universo. A inflação termina quando o campo passa ao mínimo absoluto do potencial por meio de um processo de tunelamento. Como o próprio Guth salientou [2], esta transição não poderia ocorrer nem completa e tampouco suavemente, o problema sendo evitado no cenário da inflação nova $[4,3]$, no qual adiciona-se uma fase durante a qual o campo rola lentamente ao longo de seu potencial.

Atualmente há uma grande variedade de modelos inflacionários (ver, por exemplo, [63] para uma revisão recente): $R^{2}$, inflação nova, caótica, extendida, por lei de potência, híbrida, natural, super-natural, extra-natural, eterna, termo $D$, termo $F$, de branas, $k$-inflação, fantasma, tachyon, etc. A título de exemplo, vamos considerar nesta seção a inflação caótica de Linde [3, 64], na qual podemos implementar o chamado regime de rolamento lento durante o qual são geradas as perturbações de densidade responsáveis pela formação de estruturas de grandes escalas. 


\subsection{Dinâmica inflacionária}

Uma maneira simples de se implementar um modelo inflacionário é introduzindo um campo escalar real $\phi$, chamado de inflaton, como fonte de matéria, e que evolui sob a ação de um potencial $V(\phi)$. Essa idéia é também bem motivada do ponto de vista teórico, uma vez que campos escalares são ingredientes fundamentais em teorias modernas da física de partículas. Sua ação é dada por

$$
S=-\int \sqrt{-g} d^{4} x\left[\frac{1}{2} \partial^{\mu} \phi \partial_{\mu} \phi+V(\phi)\right] .
$$

Variando a ação com relação à métrica obtemos o tensor de momento e energia para $\phi$ :

$$
T_{\nu}^{\mu}=\partial^{\mu} \phi \partial_{\nu} \phi-\delta_{\nu}^{\mu}\left(\frac{1}{2} \partial^{\lambda} \phi \partial_{\lambda} \phi+V(\phi)\right) .
$$

Para um campo homogêneo $\phi=\phi(t)$, desprezamos os gradientes espaciais, de tal modo que o inflaton pode efetivamente ser descrito como sendo um fluido perfeito cujas densidade de energia e pressão são dadas por

$$
\begin{aligned}
\rho & =\frac{1}{2} \dot{\phi}^{2}+V(\phi) \\
P & =\frac{1}{2} \dot{\phi}^{2}-V(\phi)
\end{aligned}
$$

A dinâmica do inflaton fica então completamente determinada ao substituirmos as relações acima nas equações de Friedmann. Obtemos

$$
\begin{aligned}
H^{2} & =\frac{8 \pi G}{3}\left[\frac{1}{2} \dot{\phi}^{2}+V(\phi)\right] \\
\ddot{a} & =-\frac{8 \pi G}{3}\left[\dot{\phi}^{2}-V(\phi)\right]
\end{aligned}
$$

Nas equações acima já desprezamos o termo de curvatura, uma vez que uma fase de expansão acelerada suficientemente longa levará este termo tender rapidamente a zero.

A conservação do tensor de momento e energia do inflaton, por sua vez, implica a seguinte equação de Klein-Gordon para $\phi$ :

$$
\ddot{\phi}+3 H \phi+V_{, \phi}=0
$$

onde $V_{, \phi} \equiv d V / d \phi$. 
A forma precisa do potencial deve ser guiada por uma teoria fundamental de altas energias. No entanto, como esperamos que as características gerais da inflação sejam independentes dos detalhes de $V(\phi)$, podemos então estudar a propagação do inflaton na chamada aproximação de rolamento lento em que se supõe

$$
\begin{aligned}
\dot{\phi}^{2} & \ll V(\phi) \\
\ddot{\phi} & \ll 3 H \dot{\phi}
\end{aligned}
$$

A equação (3.6) garante que a inflação provoque uma expansão do universo quase exponencial, porque, nesse caso, $w \equiv P / \rho \approx-1$. Ou seja, a equação de estado do inflaton se reduz, nesse regime, à equação de estado da constante cosmológica.

Levando-se em consideração as aproximações (3.6) e (3.7), as equações de campo se reduzem a

$$
\begin{aligned}
H^{2} & \simeq \frac{8 \pi G}{3} V(\phi) \\
3 H \dot{\phi} & \simeq-V_{, \phi}
\end{aligned}
$$

Podemos utilizar as equações do inflaton no regime de rolamento lento para derivar condições sobre a forma de $V(\phi)$. Com efeito, tomando-se a derivada em relação ao tempo de $H^{2}$ e $\dot{\phi}$ e lembrando que

$$
\dot{H}=-4 \pi G \dot{\phi}^{2}
$$

obtemos

$$
\frac{8 \pi G}{3}\left(\frac{V_{, \phi}}{V}\right)^{2} \ll 1, \frac{8 \pi G}{3}\left|\frac{V_{, \phi \phi}}{V}\right|^{2} \ll 1
$$

Uma forma bastante conveniente de se introduzir as aproximações de rolamento lento é através dos seguintes parâmetros:

$$
\epsilon \equiv-\frac{\dot{H}}{H^{2}}=4 \pi G \frac{\dot{\phi}^{2}}{H^{2}}, \delta \equiv-\frac{\ddot{\phi}}{H \dot{\phi}} .
$$

A validade das desigualdades (3.6) e (3.7) implica $\{\epsilon, \delta\} \ll 1$. Além disso, contanto que estas últimas desigualdades sejam satisfeitas, o parâmetro $\epsilon$ pode ser visto como uma constante em primeira ordem de aproximação, porque 
$\dot{\epsilon}=2 H\left(\epsilon^{2}-\epsilon \delta\right)$.

A fase de inflação termina quando $\epsilon$ se aproxima da unidade, como podemos ver da relação $\ddot{a} / a=H^{2}(1-\epsilon)>0$ que segue imediatamente da primeira equação de (3.12). Assim, é importante verificar que as condições de rolamento lento permanecem válidas por um intervalo de tempo suficientemente grande para que os problemas do horizonte e da planura possam ser resolvidos.

Uma quantidade útil que descreve o quanto deve durar a inflação é o chamado número de "e-foldings", definido por

$$
N \equiv \ln \frac{a_{f}}{a}=\int_{t}^{t_{f}} H d t \simeq 8 \pi G \int_{\phi_{f}}^{\phi} \frac{V}{V_{, \phi}} d \phi,
$$

onde o rótulo $f$ denota quantidades calculadas no final da inflação. Para que o problema da planura seja resolvido, é preciso que, logo depois do final da inflação, $\left|\Omega_{f}-1\right| \lesssim 10^{-60}$. Por outro lado, a razão de $|\Omega-1|$ entre as fases inicial e final da inflação de rolamento lento é

$$
\frac{\left|\Omega_{f}-1\right|}{\left|\Omega_{i}-1\right|} \simeq\left(\frac{a_{i}}{a_{f}}\right)^{2}=e^{-2 N_{i}},
$$

onde usamos o fato de que $H$ é aproximadamente constante entre essas fases de rolamento lento. Assumindo que $\left|\Omega_{i}-1\right|$ é da ordem da unidade, o número de $e$-foldings é então $N \gtrsim 60$.

\subsubsection{Inflação caótica}

Tipicamente, modelos inflacionários com campo escalar possuem soluções atratoras que garantem a validade das condições $\{\epsilon, \delta\} \ll 1$ por um longo período de tempo. A propriedade do modelo apresentar soluções atratoras é desejável, uma vez que a inflação pode ser vista como uma teoria de condições iniciais que "prepara" um universo plano e homogêneo, e uma dependência dos detalhes da inflação por um outro conjunto de condições iniciais iria de encontro com este propósito.

Um exemplo característico é a chamada inflação caótica, introduzida por Linde [3, 64] em 1982, e cujo potencial é

$$
V(\phi)=\frac{m^{2} \phi^{2}}{2}
$$


onde $m$ é a massa do campo. Nesse cenário o campo inicia sua evolução rolando lentamente através do potencial. Nessa fase a condição (3.6) é satisfeita e o fator de escala cresce exponencialmente. Quando o campo se aproxima do mínimo do potencial, a inflação chega a seu fim. O inflaton passa então a oscilar em torno do ponto de mínimo, processo no qual sua energia é convertida em outras partículas que reaquecerão o universo, levando a inflação à era da radiação.

$\mathrm{Na}$ aproximação de rolamento lento, as equações (3.8) e (3.9) resultam em

$$
\begin{gathered}
\phi=\phi_{i}-\frac{m}{2 \sqrt{3 \pi G}} t, \\
a(t)=a_{i} \exp \left[2 m \sqrt{\frac{\pi G}{3}}\left(\phi_{i} t-\frac{m}{4 \sqrt{3 \pi G}} t^{2}\right)\right],
\end{gathered}
$$

onde $\phi_{i}$ e $a_{i}$ correspondem aos valores iniciais do campo e do fator de escala. A equação (3.17) nos diz que o universo se expande exponencialmente no início da inflação, e que a taxa de expansão é ralentada à medida que o segundo termo entre colchetes se torna mais importante. De posse destes resultados, podemos agora estimar o valor mínimo de $\phi$ consistente com a aproximação de rolagem lenta, assim como seu valor inicial levando-se em conta o número de "e-foldings" tal que $N \gtrsim 60$. Para o potencial (3.15), temos

$$
\epsilon=\left(4 \pi G \phi^{2}\right)^{-1} \lesssim 1
$$

Ou seja, o valor mínimo de $\phi$ compatível com o regime de rolagem lenta é

$$
\phi \gtrsim \sqrt{\frac{1}{4 \pi G}} \equiv \frac{m_{P l}}{\sqrt{4 \pi}},
$$

onde $m_{P l}^{2} \equiv 1 / G$ é a massa de Planck. Usando agora a definição do número de "e-foldings" (3.13), obtemos

$$
N=\frac{2 \pi \phi_{i}^{2}}{m_{P l}^{2}}-\frac{1}{2} \gtrsim 60
$$

ou seja, $\phi_{i} \gtrsim 3 m_{P l}$. 


\subsection{Perturbações de densidade}

É um fato experimental que o universo não é completamente homogêneo nem isotrópico. Na realidade, o princípio cosmológico é válido apenas em média, ou melhor, em uma média em escalas de distância da ordem de algumas centenas de megaparsecs. Em escalas menores o que observamos é uma miríade de estruturas complexas e assimétricas tais como galáxias, aglomerados de galáxias, "vazios" e filamentos de matéria. Portanto, para termos uma descrição mais refinada de nosso universo, devemos necessariamente ir além do modelo homogêneo e isotrópico de FRW através do estudo de pequenos desvios nas distribuições de matéria e da geometria do espaço-tempo.

Nesta descrição não-homogênea, a métrica pode ser escrita como

$$
g_{\mu \nu}=\bar{g}_{\mu \nu}(\eta)+\delta g_{\mu \nu}(\eta, x)
$$

onde quantidades de fundo são escritos com uma barra superior e $\eta$ é o tempo conforme definido como $d \eta \equiv d t / a$. Explicitamente, temos

$$
\delta g_{\mu \nu}=\left(\begin{array}{cc}
-2 A & B_{, i} \\
B_{, i} & -2\left(\psi \delta_{i j}-E_{, i j}\right)
\end{array}\right)
$$

Em (3.48), estamos considerando apenas o setor escalar das perturbações, que são as perturbações de interesse que vão se acoplar com as perturbações da matéria. No que segue, uma "linha" denotará derivada em relação ao tempo conforme.

Substituindo a expressão (3.48) nas equações de Einstein e coletando termos de primeira ordem nos campos de flutuação obtemos

$$
\delta G_{\mu \nu}=8 \pi G \delta T_{\mu \nu}
$$

É importante salientar aqui o fato de que $\delta G_{\mu \nu}$ e $\delta T_{\mu \nu}$ não são objetos invariantes de calibre. Em uma abordagem invariante, no entanto, é sempre 
possível [65] construir objetos invariantes de calibre através de

$$
\begin{aligned}
\delta G_{0}^{(i c) 0} & \equiv \delta G_{0}^{0}+\left(\left(\bar{G}_{0}^{0}\right)^{\prime}\right)\left(B-E^{\prime}\right) \\
\delta G_{i}^{(i c) 0} & \equiv \delta G_{i}^{0}+\left(\bar{G}_{i}^{0}-\frac{1}{3} \bar{G}_{k}^{k}\right)\left(B-E^{\prime}\right)_{, i} \\
\delta G_{j}^{(i c) i} & \equiv \delta G_{j}^{i}+\left(\left(\bar{G}_{j}^{i}\right)^{\prime}\right)\left(B-E^{\prime}\right)
\end{aligned}
$$

De modo análogo, podemos também definir objetos invariantes de calibre $\delta T_{\mu \nu}^{(i c)}$ lineares nas perturbações da métrica e do campo. Outras duas quantidades invariantes de calibre de interesse são os potenciais de Bardeen [66], definidos por

$$
\begin{aligned}
\Phi & \equiv A+\frac{1}{a}\left[\left(B-E^{\prime}\right) a\right]^{\prime} \\
\Psi & \equiv \psi-\frac{a^{\prime}}{a}\left(B-E^{\prime}\right) .
\end{aligned}
$$

No calibre longitudinal, ou seja, quando $B=0=E$, temos $\Phi=A$ e $\Psi=\psi$.

Em termos dos tensores $\delta G_{\mu}^{(i c) v}$ e $\delta T_{\mu}^{(i c) v}$ as equações de movimento lineares nas flutuações dos campos ficam

$$
\delta G_{\mu \nu}^{(i c)}=8 \pi G \delta T_{\mu \nu}^{(i c)}
$$

Ao inserir nas equações acima as expressões para a métrica e para o campo, equações (3.48) e (3.30) (que são elas mesmas dependentes de calibre), terminaremos apenas com combinações invariantes de calibre das variáveis de flutuação.

Em uma abordagem não-invariante, podemos obviamente fixar um calibre desde o começo. No calibre longitudinal, a métrica fica

$$
d s^{2}=a^{2}\left[-(1+2 A) d \eta^{2}+(1-2 \psi) \delta_{i j} d x^{i} d x^{j}\right]
$$

O passo seguinte para se obter as equações de movimento é simplesmente substituir o "ansatz" (3.26) nas equações (3.21).

Em ambos os casos obtemos as seguintes equações de movimento lineari- 
zadas:

$$
\begin{aligned}
-3 \mathcal{H}\left(\mathcal{H} \Phi+\Psi^{\prime}\right)+\nabla^{2} \Psi & =4 \pi G a^{2} \delta T_{0}^{(i c) 0} \\
\left(\mathcal{H} \Phi+\Psi^{\prime}\right)_{, i} & =4 \pi G a^{2} \delta T_{i}^{(i c) 0} \\
{\left[\left(2 \mathcal{H}^{\prime}+\mathcal{H}^{2}\right) \Phi+\mathcal{H} \Phi^{\prime}+\Psi^{\prime \prime}+2 H \Psi^{\prime}\right] \delta_{j}^{i} } & \\
+\frac{1}{2} \nabla^{2} D \delta_{j}^{i}-\frac{1}{2} \delta^{i k} D_{, k j} & =-4 \pi G a^{2} \delta T_{j}^{(i c) i}
\end{aligned}
$$

onde $D \equiv \Phi-\Psi$ e $\mathcal{H} \equiv a^{\prime} / a$. No calibre longitudinal, temos $\Phi=A, \Psi=\psi$ e $\delta T_{j}^{(i c) i}=\delta T_{j}^{i}$.

Uma importante conclusão é que para fluidos de matéria sem tensão anisotrópica em primeira ordem nos campos de flutuação, ou seja, quando $\delta T_{j}^{i}=0$ para $i \neq j$, vale a igualdade

$$
\Phi=\Psi
$$

Isto vale para muitos casos de interesse como, por exemplo, em teorias cuja matéria é descrita por campos escalares canônicos e para fluidos perfeitos sem tensão anisotrópica.

Vamos agora fixar nossa atenção para o caso em que a matéria é descrita por um campo escalar, cuja ação é dada por

$$
S=\int d^{4} x \sqrt{-g}\left[-\frac{1}{2} \phi^{, \alpha} \phi_{, \alpha}-V(\phi)\right]
$$

$\phi$ é dado pela expansão

$$
\phi(\eta, x)=\bar{\phi}(\eta)+\delta \phi(\eta, x)
$$

Elegendo o calibre longitudinal, as equações (3.27) se reduzem a

$$
\begin{aligned}
\nabla^{2} \psi-3 \mathcal{H} \psi^{\prime}-\left(\mathcal{H}^{\prime}+2 \mathcal{H}^{2}\right) \psi & =4 \pi G\left(\bar{\phi}^{\prime} \delta \phi^{\prime}+V^{\prime} a^{2} \delta \phi\right) \\
\psi^{\prime}+\mathcal{H} \psi & =4 \pi G \bar{\phi}^{\prime} \delta \phi \\
\psi^{\prime \prime}+3 \mathcal{H} \psi^{\prime}+\left(\mathcal{H}^{\prime}+2 \mathcal{H}^{2}\right) \psi & =4 \pi G\left(\bar{\phi}^{\prime} \delta \phi^{\prime}-V^{\prime} a^{2} \delta \phi\right)
\end{aligned}
$$

Apesar da aparente complexidade deste sistema de equações, manipulações imediatas mostram que tudo pode ser reduzido a uma única equação mestra, 
que é

$$
\psi_{k}^{\prime \prime}+2\left(\mathcal{H}-\frac{\bar{\phi}^{\prime \prime}}{\bar{\phi}^{\prime}}\right) \psi_{k}^{\prime}+\left[k^{2}+2\left(\mathcal{H}^{\prime}-\mathcal{H} \frac{\bar{\phi}^{\prime \prime}}{\bar{\phi}^{\prime}}\right)\right] \psi_{k}=0
$$

já escrita no espaço dos momentos.

$\mathrm{Na}$ equação (3.32) o último termo corresponde a um termo de força que gera instabilidades, enquanto que o antepenúltimo leva a soluções oscilatórias. O segundo termo, por sua vez, corresponde a um termo de fricção que vai contribuir para o amortecimento da amplitude de oscilação e compensar a instabilidade gerada pelo último termo. Para cada número de onda $k$, há duas soluções fundamentais: em pequenas escalas $(k>H)$, as soluções correspondem a oscilações amortecidas; em grandes escalas $(k<H)$, as soluções se congelam e a propagação do modo é controlada pelos termos de força e de fricção.

Uma conseqüência relevante da equação (3.32) torna-se clara ao introduzirmos a variável $\mathcal{R}$ definida como

$$
\mathcal{R}=\psi+\frac{\mathcal{H}}{4 \pi G \bar{\phi}^{\prime 2}}\left(\psi^{\prime}+H \psi\right)
$$

Vamos escrevê-la sob uma forma conveniente usando a equação (3.3) e lembrando que $P=w \rho$. Nesse caso,

$$
\mathcal{R}=\psi+\frac{2}{3}\left(\frac{H^{-1} \dot{\psi}+\psi}{1+w}\right)
$$

Em termos de $\mathcal{R}$, a equação mestra (3.32) fica

$$
\frac{3}{2} \dot{\mathcal{R}} H(1+w)=0+\mathcal{O}\left(k^{2} \psi\right)
$$

O segundo termo do lado direito pode ser ignorado em largas escalas, de tal modo que

$$
\dot{\mathcal{R}}(1+w)=0
$$

Logo, se $1+w \neq 0$ (como ocorre no caso inflacionário quando o inflaton passa a oscilar [67]), a variável $\mathcal{R}$ se conserva em regimes de grandes comprimentos de onda. Além disso, é possível mostrar em modelos com um único campo [68] que $\mathcal{R}=$ constante em escalas super-Hubble independentemente das hi- 
póteses assumidas sobre a equação de estado. Levando-se em conta esta lei de conservação, podemos assim relacionar a amplitude do potencial gravitacional em épocas distintas da evolução do universo, por exemplo, entre as épocas nas quais o modo cruza o horizonte de Hubble.

\subsection{Invariância do espectro primordial}

Uma importante previsão da teoria inflacionária diz respeito à invariância do espectro de potência das flutuações de densidade primordiais, definido como sendo a função de Green, calculada em tempos iguais, da transformada de Fourier de $\mathcal{R}(\eta, \boldsymbol{x})$. Explicitamente,

$$
\mathcal{P}_{\mathcal{R}} \equiv \frac{k^{3}}{2 \pi^{2}}\left|\mathcal{R}_{k}^{2}\right|
$$

O espectro de potência pode ser confrontado com as observações de anisotropias da radiação cósmica de fundo, sendo, para esse efeito, convenientemente parametrizado em termos do índice espectral $n_{S}$ definido como

$$
n_{S}-1 \equiv \frac{d \ln \mathcal{P}_{\mathcal{R}}}{d \ln k}
$$

Para vermos isso em mais detalhes, é suficiente considerarmos o modelo de inflação de campo único apresentado no início deste capítulo. Com esse propósito, vamos escrever a equação mestra (3.32) em termos das novas variáveis $v$ e $z$ definidas como:

$$
\begin{aligned}
v & =a\left[\delta \phi+\frac{\bar{\phi}^{\prime}}{\mathcal{H}} \psi\right]=z \mathcal{R} \\
z & =\frac{a \bar{\phi}^{\prime}}{\mathcal{H}}
\end{aligned}
$$

$v$ é conhecido como variável de Mukhanov-Sasaki. Em teoria quântica de perturbações cosmológicas [65], identifica-se naturalmente $v$ como sendo a variável canônica de quantização.

Nessa representação, a equação mestra fica

$$
v_{k}^{\prime \prime}+\left(k^{2}-\frac{z^{\prime \prime}}{z}\right) v_{k}=0
$$


também conhecida como equação de Mukhanov-Sasaki. Em inflação caótica em regime de rolamento lento, $\mathcal{H}$ e $\bar{\phi}^{\prime}$ são proporcionais (ver equações (3.16) e (3.17)). Logo, $z \simeq a$. Portanto, o termo de massa da equação (3.40) é dado pela escala de Hubble $H^{2}$, ou seja, $k_{H} \equiv z^{\prime \prime} / z \sim H^{2}$. Quando $k>k_{H}$, as soluções para $v_{k}$ são oscilações de amplitude constante. Em $k=k_{H}$, ou seja, quando o modo cruza a escala de Hubble, essas oscilações são inibidas. Para $k<k_{H}$ as soluções para $v_{k}$ crescem com $z, v_{k} \sim z$.

Durante a inflação, os parâmetros de rolamento lento obedecem a $\{\epsilon, \delta\} \ll$ 1, além de serem constantes em primeira ordem de aproximação. Levando tudo isso em conta, obtemos

$$
\eta \simeq \frac{1+\epsilon}{a H}, \frac{z^{\prime \prime}}{z} \simeq \frac{\nu^{2}-1 / 4}{\eta^{2}}, \nu \equiv \frac{3}{2}+3 \epsilon+\delta
$$

de tal forma que a equação de Mukhanov-Sasaki se reduz a uma equação de Bessel com solução geral dada por

$$
v_{k}=\frac{\sqrt{\pi|\eta|}}{2} e^{\frac{i(1+2 \nu) \pi}{4}}\left[c_{1} H_{\nu}^{(1)}(k|\eta|)+c_{2} H_{\nu}^{(2)}(k|\eta|)\right]
$$

A escolha do vácuo de Minkowski ${ }^{1}$

$$
v_{k} \rightarrow \frac{e^{-i k \eta}}{\sqrt{2 k}}
$$

como estado inicial em $-k \eta \rightarrow \infty$ implica $c_{1}=1$ e $c_{2}=0$ em (3.42). Logo, em largas escalas $(k \ll a H)$, obtemos

$$
\mathcal{P}_{\mathcal{R}}=\left[(1-\epsilon) \frac{\Gamma(\nu)}{\Gamma\left(\frac{3}{2}\right)} \frac{H^{2}}{2 \pi \dot{\bar{\phi}}}\right]^{2}\left(\frac{|k \eta|}{2}\right)^{3-2 \nu}
$$

onde usamos $\lim _{k \eta \rightarrow 0} H_{\nu}^{(1)}(k|\eta|)=-(i / \pi) \Gamma(\nu)(k|\eta| / 2)^{-\nu}$ e $\Gamma(3 / 2)=\sqrt{\pi} / 2$. Em particular, para um campo escalar sem massa em um espaço-tempo de de Sitter, $\epsilon=0=\delta$ exatamente. Obtemos nesse caso o conhecido espectro puro de Harrison-Zel'dovich:

$$
\mathcal{P}_{\mathcal{R}}=\left(\frac{H^{2}}{2 \pi \dot{\bar{\phi}}}\right)^{2}, k \ll a H .
$$

\footnotetext{
${ }^{1}$ Escolha esta que será justificada na próxima seção.
} 
Em virtude da conservação de $\mathcal{R}$ em largas escalas, podemos fixar a amplitude inicial do espectro no instante em que o modo cruza o horizonte pela primeira vez, em $k=a H$. Desse modo,

$$
\mathcal{P}_{\mathcal{R}} \simeq\left(\frac{H^{2}}{2 \pi \dot{\bar{\phi}}}\right)_{k=a H}^{2}
$$

em ordem dominante nos parâmetros de rolamento lento.

Podemos agora usar as equações (3.8) e (3.9) para escrever o resultado acima em termos do potencial com o intuito de estimar a escala de energia da inflação. Nesse caso,

$$
\mathcal{P}_{\mathcal{R}}=\left(\frac{128 \pi}{3 m_{P l}^{6}} \frac{V^{3}}{V_{, \phi}^{2}}\right)_{k=a H} .
$$

A normalização dada pelo satélite COBE [69] corresponde a $\mathcal{P}_{\mathcal{R}} \simeq 2 \times 10^{-9}$ para o modo que cruzou o horizonte 60 "e-foldings" antes do fim da inflação. Consideremos o modelo caótico, com $V=m_{\phi}^{2} \phi^{2} / 2$. Vimos que o valor do campo 60 "e-foldings" antes do fim da inflação é $\phi \simeq 3 m_{P l}$. Usando esse valor para calcular o potencial e levando-se em conta a normalização do COBE, obtem-se $m_{\phi} \simeq 10^{-6} m_{P l}$.

O índice espectral é

$$
n_{S}=1-6 \epsilon+2 \delta .
$$

Como durante a inflação de rolagem lenta vale $\{\epsilon, \delta\} \ll 1$, o espectro de perturbações de densidade gerado durante a inflação é quase invariante de escala. O espectro é dito ser "inclinado para o vermelho" se $n_{S}<1$, e "inclinado para o azul" se $n_{S}>1$.

Consideremos novamente o potencial caótico quadrático. Nesse caso,

$$
n_{S}=1-\frac{1}{8 \pi}\left(\frac{m_{P l}}{\phi}\right)^{2}
$$

ou seja, um espectro inclinado para o vermelho. Vemos assim que, essencialmente, a dependência de escala eventualmente adquirida pelo espectro está relacionada com o fato do inflaton ser ou não ser massivo.

No modelo inflacionário de rolamento lento com um campo escalar, o índice espectral não pode ser muito menor (ou maior) que a unidade. Esta previsão concorda com o quinto ano de resultados do satélite WMAP [1], cujo resultado 
para o índice espectral é $n_{S}=0,96_{-0,013}^{+0,014}$ com $68 \%$ de nível de confiança.

\subsection{Quantização de perturbações de densidade}

Esta seção é dedicada a um importante aspecto da teoria inflacionária, que é a quantização de perturbações. Do tratamento clássico da seção anterior, extraindo graus de liberdade espúrios por uma fixação de calibre, vimos que o estudo das flutuações de densidade da métrica pode ser reduzido ao estudo da evolução de uma única variável (que é a variável de Mukhanov-Sasaki). No caso quântico é portanto de se esperar que a teoria possa também ser reduzida a uma única variável dinâmica, que é, novamente, a variável $v$. Como o espaçotempo de fundo no qual este campo escalar evolui é dependente do tempo, a massa de $v$ também será dependente do tempo (a massa de $v$ corresponde à razão $\left.z^{\prime \prime} / z\right)$. Em conseqüência disto, haverá uma produção de partículas a partir do estado de vácuo de $v$. Devido à evolução do espaço-tempo de fundo, os comprimentos de onda dos modos são estirados a escalas maiores que o horizonte de Hubble, voltando a cruzá-lo em um tempo cósmico futuro, gerando instabilidades que vão desencadear a formação de estruturas. Portanto, a teoria quântica de perturbações cosmológicas oferece um quadro consistente no qual se pode compreender tanto a origem como a evolução das flutuações cosmológicas.

Para obtermos a ação para as perturbações de densidade, começamos a análise a partir de

$$
S=\frac{1}{16 \pi G} \int d^{4} x \sqrt{-g}\left[R-\frac{1}{2} \phi^{, \alpha} \phi_{, \alpha}+V(\phi)\right]
$$

É conveniente eleger um calibre desde o início, que é o calibre longitudinal, sob o qual a métrica assume a seguinte forma:

$$
d s^{2}=a^{2}(\eta)\left[-(1+2 \psi) d \eta^{2}+(1-2 \psi) d x^{2}\right] .
$$

Note que na expressão (3.48) já levamos em conta o fato de que não há tensão anisotrópica em teorias com campo escalar canônico. O campo escalar, por sua vez, é expandido como

$$
\phi(\eta, \boldsymbol{x})=\bar{\phi}(\eta)+\delta \phi(\eta, \boldsymbol{x})
$$


Devemos agora substituir as expressões (3.48) e (3.49) em (3.47) e expandí-la até segunda ordem nos campos de flutuação. Este cálculo é longo e complicado, porém assaz conhecido [65]. Logo, vamos aqui nos restringir a reproduzir o resultado, no qual fica óbvia a escolha de $v$ como variável canônica:

$$
S^{(2)}=\frac{1}{2} \int d^{4} x\left[v^{\prime 2}-v_{, i} v_{, i}+\frac{z^{\prime \prime}}{z} v^{2}\right]
$$

O momento canonicamente conjugado a $v$ é

$$
\pi=\frac{\delta S^{(2)}}{\delta v^{\prime}}=v^{\prime}
$$

Em teoria quântica, as variáveis clássicas são promovidas a operadores $\hat{v}(\eta, \boldsymbol{x})$ e $\hat{\pi}(\eta, \boldsymbol{y})$ sujeitos às seguintes regras de comutação em tempos iguais:

$$
\begin{aligned}
{[\hat{v}(\eta, \boldsymbol{x}), \hat{\pi}(\eta, \boldsymbol{y})] } & =i \delta^{(3)}(\boldsymbol{x}-\boldsymbol{y}) \\
{[\hat{v}(\eta, \boldsymbol{x}), \hat{v}(\eta, \boldsymbol{y})] } & =0 \\
{[\hat{\pi}(\eta, \boldsymbol{x}), \hat{\pi}(\eta, \boldsymbol{y})] } & =0
\end{aligned}
$$

Podemos escrever os operadores $\hat{v}(\eta, \boldsymbol{x})$ e $\hat{\pi}(\eta, \boldsymbol{y})$ em termos de operadores de criação e aniquilação do espaço de Fock, $\hat{a}_{\boldsymbol{k}}$ e $\hat{a}_{\boldsymbol{k}}^{\dagger}$, como

$$
\begin{aligned}
\hat{v}(\eta, \boldsymbol{x}) & =\int \frac{d^{3} k}{(2 \pi)^{3 / 2}}\left(v_{k}(\eta) \hat{a}_{\boldsymbol{k}} e^{i \boldsymbol{k} \cdot \boldsymbol{x}}+v_{k}^{*}(\eta) \hat{a}_{\boldsymbol{k}}^{\dagger} e^{-i \boldsymbol{k} \cdot \boldsymbol{x}}\right) \\
\hat{\pi}(\eta, \boldsymbol{x}) & =\int \frac{d^{3} k}{(2 \pi)^{3 / 2}}\left(\pi_{k}(\eta) \hat{a}_{\boldsymbol{k}} e^{i \boldsymbol{k} \cdot \boldsymbol{x}}+\pi_{k}^{*}(\eta) \hat{a}_{\boldsymbol{k}}^{\dagger} e^{-i \boldsymbol{k} \cdot \boldsymbol{x}}\right)
\end{aligned}
$$

Os operadores de criação e aniquilação devem obedecer às regras de comutação

$$
\begin{aligned}
{\left[\hat{a}_{\boldsymbol{k}}, \hat{a}_{\boldsymbol{q}}^{\dagger}\right] } & =\delta^{(3)}(\boldsymbol{k}-\boldsymbol{q}) \\
{\left[\hat{a}_{\boldsymbol{k}}, \hat{a}_{\boldsymbol{q}}\right] } & =0 \\
{\left[\hat{a}_{\boldsymbol{k}}^{\dagger}, \hat{a}_{\boldsymbol{q}}^{\dagger}\right] } & =0
\end{aligned}
$$

e definem o estado de vácuo como sendo o vetor $|0\rangle$ do espaço de Fock tal que

$$
\hat{a}_{\boldsymbol{k}}|0\rangle=0
$$

Em vista das regras de comutação (3.52) e (3.55), temos também a seguinte 
condição de normalização

$$
v_{k}(\eta) v_{k}^{*}(\eta)-v_{k}^{\prime}(\eta) v_{k}^{*}(\eta)=i
$$

que nos permite fixar as amplitudes das perturbações univocamente e de maneira compatível com o princípio da incerteza de Heisenberg.

O próximo passo a ser dado no processo de quantização das perturbações diz respeito à determinação do estado inicial. Como flutuações presentes em um universo inflacionário são todas estiradas para o vermelho, o que se assume é que, em um tempo inicial $t_{i}$, o campo $v$ iniciou sua evolução, modo por modo, em seu estado de vácuo. Devemos então especificar $t_{i}$ assim como um vácuo entre vários possíveis vácuos. Do fato do modo oscilar com amplitude constante em escalas inferiores à escala de Hubble, segue então que a escolha de $t_{i}$ tornase irrelevante. O estado de vácuo, por sua vez, é usualmente escolhido como sendo o vácuo de Minkowski, uma vez que esse estado é livre de partículas. Logo, o estado inicial é dado por

$$
v_{k} \rightarrow \frac{e^{-i k \eta}}{\sqrt{2 k}}, k \eta \rightarrow-\infty
$$

Portanto, em teoria quântica de perturbações cosmológicas, as perturbações são definidas modo por modo em um instante inicial $t_{i}$ em seus estados de vácuo (vazio). Enquanto seus comprimentos de onda são menores que a escala de Hubble, o estado sofre flutuações quânticas de vácuo. Por causa da expansão do espaço-tempo de fundo, os comprimentos de onda são estirados até cruzarem o raio de Hubble, instante no qual as oscilações são congeladas. Em escalas super-Hubble, a amplitude das perturbações cresce com o fator de escala. Ao cruzarem novamente o horizonte em um tempo futuro, as flutuações emergem como perturbações clássicas responsáveis pela formação de estruturas em largas escalas.

\subsection{Comentários}

Embora o modelo cosmológico padrão ofereça uma descrição consistente da história de nosso universo desde a era da nucleossíntese até o presente, duas questões intrínsecas permanecem sem resposta: por que a radiação cósmica de fundo é isotrópica em escalas bem maiores que o horizonte e por que o universo 
atual é plano.

A teoria inflacionária oferece uma solução dinâmica para esses problemas usando relatividade geral e campos escalares semi-classicamente. Oferece também um mecanismo causal para explicar a origem e evolução das flutuações primordiais. Do ponto de vista observacional, uma importante previsão da inflação é o espectro de perturbações de densidade de origem quântica, cuja forma é perfeitamente consistente com as observações.

Com todos os seus sucessos, podemos nos perguntar qual seria a razão em procurar por teorias alternativas ao paradigma inflacionário.

A primeira razão é simples: embora a inflação possa ser implementada em teorias de física de partículas de altas energias [70], ela não é a única possibilidade que delas emerge. Portanto, é legítimo que se investigue todas as outras com o intuito de se comparar suas previsões. Observações futuras certamente desempenharão o papel de selecionar o bom modelo.

A segunda razão agrupa problemas intrínsicos à própria inflação:

- ela não aborda e não resolve a questão da singularidade inicial [7], ao contrário do que se esperaria de um modelo do universo primordial.

- Muitos modelos de inflação são baseados em uma descrição da matéria em termos de campos escalares, campos estes jamais observados na natureza.

- Por último, podemos mencionar o fato de que a dinâmica inflacionária é governada pelas equações da relatividade geral em escalas planckianas (vimos que a massa do inflaton é da ordem de $10^{-6} m_{P l}$ ), ou seja, em um regime onde efeitos quânticos se tornam importantes e onde não mais se garante a validade de suas equações. O mesmo tipo de problema está relacionado com a escolha de condições iniciais, para as quais o comprimento de onda pode ser menor do que o próprio comprimento de Planck [71].

Historicamente, uma alternativa seria o cenário de pré-big bang [14], assim como cenários "ekpiróticos" [16], ambos baseados em teoria de cordas. Como veremos, são modelos que dificilmente podem acomodar espectros invariantes de escala. Uma característica em comum entre eles é o fato de apresentarem uma fase de contração seguida de expansão (no quadro de Einstein), ou seja, um ricochete [72]. Portanto, é natural que consideremos, como uma alternativa 
geral à inflação, ricochetes cosmológicos, uma idéia curiosamente muito mais velha que a própria inflação, remontando ao início da década de 30 [73, 74]. No próximo capítulo vamos estudar o primeiro ponto apontado acima sobre o problema da singularidade inicial no modelo de FRW. No capítulo seguinte passaremos ao estudo de ricochetes cosmológicos. 


\section{Capítulo 4}

\section{Singularidades}

Antes dos trabalhos de S. Hawking e R. Penrose [75], acreditava-se que a singularidade do modelo cosmológico homogêneo e isotrópico (assim como a singularidade em $r=0$ na solução de Schwarzschild ) era justamente devida ao alto grau de simetria do espaço [76, 77]. No entanto, este problema mostrou-se mais abrangente. De fato, uma conseqüência dos teoremas de singularidade [75] é que um universo em expansão no qual $(i)$ o tensor de momento e energia satisfaz à condição de energia forte $(\rho+3 P \geq 0)$ e $(i i)$ no qual o tensor de rotação que caracteriza a congruência de geodésicas é nulo, é singular em um tempo finito passado, instante no qual a densidade de energia, assim como a pressão, vão a infinito. Na seção seguinte, vamos revisar as condições de energia da relatividade geral, uma vez que são importantes como hipóteses dos teoremas de singularidade, e comentar de forma sucinta sobre as técnicas globais desenvolvidas por Hawking e Penrose necessárias para provar esses teoremas ${ }^{1}$.

\subsection{Condições de energia}

Requisitos sobre propriedades de uma distribuição de matéria qualquer, tais como positividade da densidade de energia ou o domínio desta sobre a pressão, podem ser formulados em termos de condições sobre o tensor de momento e energia. Para enunciarmos essas condições de forma concreta, é conveniente

\footnotetext{
${ }^{1}$ Este capítulo é baseado nas referências $[78,79,80,81,82]$.
} 
assumirmos que o tensor de momento e energia pode ser decomposto como

$$
T^{\alpha \beta}=\rho \hat{e}_{0}^{\alpha} \hat{e}_{0}^{\beta}+P_{1} \hat{e}_{1}^{\alpha} \hat{e}_{1}^{\beta}+P_{2} \hat{e}_{2}^{\alpha} \hat{e}_{2}^{\beta}+P_{3} \hat{e}_{3}^{\alpha} \hat{e}_{3}^{\beta}
$$

Nesta expressão $\rho$ é a densidade de energia do fluido, $P_{i}, i=1,2,3$, é a pressão e $\hat{e}_{\mu}^{\alpha}$ é uma base ortonormal que satisfaz

$$
\eta_{\mu \nu}=\hat{e}_{\mu}^{\alpha} \hat{e}_{\nu}^{\beta} g_{\alpha \beta}
$$

e $\eta_{\mu \nu}$ é o tensor métrico de Minkowski, $\eta_{\mu \nu}=\operatorname{diag}(-1,1,1,1)$. Para um fluido perfeito, $P=P_{1}=P_{2}=P_{3}$. Com a identificação $\hat{e}_{0}^{\alpha}=U^{\alpha}$, onde $U^{\alpha}$ é a quadrivelocidade do fluido, vemos que a equação (4.1) se reduz à representação usual do tensor de momento e energia de um fluido perfeito, equação (2.13).

A condição de energia fraca estabelece que a densidade de energia de uma distribuição de matéria, como vista por um observador com quadrivelocidade $v^{\alpha}$, deve ser não-negativa, ou seja, que

$$
T_{\alpha \beta} v^{\alpha} v^{\beta} \geq 0
$$

Escrevendo $v^{\alpha}$ como

$$
v^{\alpha}=\gamma\left(\hat{e}_{0}^{\alpha}+a \hat{e}_{1}^{\alpha}+b \hat{e}_{2}^{\alpha}+c \hat{e}_{3}^{\alpha}\right),
$$

onde $a, b$ e $c$ são funções arbitrárias das coordenadas, $\gamma=\left(1-a^{2}-b^{2}-c^{2}\right)^{-1 / 2}$ e $a^{2}+b^{2}+c^{2}<1$, obtemos da condição $T_{\alpha \beta} v^{\alpha} v^{b} \geq 0$ e da equação (4.1) que

$$
\rho+a^{2} P_{1}+b^{2} P_{2}+c^{2} P_{3} \geq 0 .
$$

Escolhendo os fatores arbitrários $a, b$ e $c$ tais que $0=a=b=c$, obtemos $\rho \geq 0$. Alternativamente, se $0=b=c$, então $\rho+a^{2} P_{1} \geq 0$. Nesse caso, como $a^{2}<1$, então $0 \leq \rho+a^{2} P_{1}<\rho+P_{1}$, ou, $\rho+P_{1} \geq 0$. Relações similares valem para $0=a=c$ e $0=a=b$. Portanto, a condição de energia fraca implica

$$
\begin{aligned}
\rho & \geq 0 \\
\rho+P_{i} & \geq 0
\end{aligned}
$$

onde $i=1,2,3$.

A condição de energia nula é similar à condição de energia fraca, com a 
diferença de que o quadrivetor $v^{\alpha}$ (do tipo tempo) é substituído por um quadrivetor nulo $k^{\alpha}$ direcionado para o futuro. De forma concreta, essa condição estabelece que

$$
T_{\alpha \beta} k^{\alpha} k^{\beta} \geq 0
$$

Decompondo $k^{\alpha}$ como

$$
k^{\alpha}=\hat{e}_{0}^{\alpha}+a^{\prime 2} \hat{e}_{1}^{\alpha}+b^{\prime 2} \hat{e}_{2}^{\alpha}+c^{\prime 2} \hat{e}_{3}^{\alpha},
$$

onde $a^{\prime 2}+b^{\prime 2}+c^{\prime 2}=1$ (pois $k^{\alpha} k_{\alpha}=0$ ), obtemos, de forma análoga ao caso anterior,

$$
\rho+P_{i} \geq 0
$$

A condição de energia forte estabelece que

$$
\left(T_{\alpha \beta}-\frac{1}{2} g_{\alpha \beta} T\right) v^{\alpha} v^{\beta} \geq 0
$$

Reescrevendo as equações de Einstein na forma

$$
R_{\mu \nu}=8 \pi G\left(T_{\mu \nu}-\frac{1}{2} g_{\mu \nu} T\right)
$$

vemos que a condição de energia forte se traduz em uma condição sobre o tensor de Ricci. Das equações (4.1) e (4.2), essa condição fica

$$
\gamma^{2}\left(\rho+a^{2} P_{1}+b^{2} P_{2}+c^{2} P_{3}\right) \geq \frac{1}{2}\left(\rho-P_{1}-P_{2}-P_{3}\right)
$$

$0=a=b=c$ implica $\gamma=1$. Logo, $\rho+\Sigma P_{i} \geq 0$. Se $0=b=c$, então $\gamma^{2}=1 /\left(1-a^{2}\right)$, ou seja, $\rho+P_{1}+P_{2}+P_{3} \geq a^{2}\left(P_{2}+P_{3}-\rho-P_{1}\right)$. Como $a^{2}<1$, devemos ter $\rho+P_{1} \geq 0$. Tomando-se $0=a=c$ e $0=a=b$, obtemos expressões similares para $P_{2}$ e $P_{3}$. Portanto, a condição de energia forte implica

$$
\begin{aligned}
\rho+\Sigma P_{i} & \geq 0 \\
\rho+P_{i} & \geq 0
\end{aligned}
$$

A condição de energia dominante reflete o fato de que o fluxo de matéria se dá ao longo de linhas de mundo do tipo tempo ou nulas. Ela estabelece que um observador com quadrivelocidade $v^{\alpha}$ medirá uma densidade de momento dado 
por $\mathcal{T}^{\alpha} \equiv-T_{\beta}^{\alpha} v^{\beta}$, onde $\mathcal{T}^{\alpha}$ é direcionado para o futuro e é do tipo tempo ou nulo. Usando novamente as equações (4.1) e (4.2), obtemos a seguinte relação:

$$
\rho^{2}-a^{2} P_{1}^{2}-b^{2} P_{2}^{2}-c^{2} P_{3}^{2} \geq 0
$$

Um cálculo similar aos casos anteriores resulta nas seguintes desigualdades:

$$
\begin{aligned}
& \rho \geq 0 \\
& \rho \geq\left|P_{i}\right|
\end{aligned}
$$

\subsection{Equação de Raychaudhuri e teorema de sin- gularidade}

A relação entre as condições de energia e propriedades globais do espaço-tempo está contida na equação de Raychaudhuri,

$$
\frac{d \theta}{d \tau}=-\frac{\theta^{2}}{3}-\sigma^{\alpha \beta} \sigma_{\alpha \beta}+\omega^{\alpha \beta} \omega_{\alpha \beta}-R_{\alpha \beta} u^{\alpha} u^{\beta},
$$

cuja solução representa a evolução da expansão escalar, usualmente denotada por $\theta$, e interpretada geometricamente como sendo a taxa de variação fracional do volume da seção transversal a uma congruência de geodésicas. No caso particular do espaço-tempo de FRW, a expansão escalar é dada por

$$
\theta=3 \frac{\dot{a}}{a}=\frac{1}{a^{3}}\left(\frac{d a^{3}}{d t}\right)
$$

Na equação (4.7), $\sigma^{\alpha \beta}$ e $\omega^{\alpha \beta}$ são os tensores de cisalhamento (com traço nulo) e de rotação (antisimétrico), respectivamente, definidos de tal forma que $\sigma^{\alpha \beta} \sigma_{\alpha \beta} \geq 0$ e $\omega^{\alpha \beta} \omega_{\alpha \beta} \geq 0$. Como tais, são interpretados em termos da deformação da área perpendicular ao fluxo geodésico. $u^{\alpha}=d x^{\alpha} / d \tau$ é o vetor tangente às geodésicas.

Um caso particular de interesse é para congruências ditas ortogonais de hipersuperfície, o que quer dizer que em todo ponto do espaço-tempo a congruência é ortogonal a uma família de hipersuperfícies do tipo espaço. Um importante teorema a esse respeito é o teorema de Frobenius, que estabelece que congruências ortogonais de superfície tem o tensor de rotação $\omega^{\alpha \beta}$ igual a zero. A congruência de linhas de mundo comóveis em um universo de FRW é 
um exemplo de congruência ortogonal de hipersuperfície.

Uma conseqüência de grande importância da equação de Raychaudhuri é revelada através do teorema da focalização geodésica: considere uma congruência de geodésicas ortogonais de superfície, de tal modo que $\omega_{\alpha \beta}=0$; se a condição de energia forte é satisfeita, ou seja, se $R_{\alpha \beta} u^{\alpha} u^{\beta} \geq 0$ (neste ponto entram em cena as equações de Einstein), então a equação de Raychaudhuri se escreve como

$$
\frac{d \theta}{d \tau}=-\frac{\theta^{2}}{3}-\sigma^{\alpha \beta} \sigma_{\alpha \beta}-R_{\alpha \beta} u^{\alpha} u^{\beta} \leq 0
$$

Logo, uma congruência inicialmente divergente $(\theta>0)$ divergirá mais lentamente no futuro, e uma congruência inicialmente convergente $(\theta<0)$ convergirá mais rapidamente no futuro. Este é o enunciado do teorema da focalização geodésica. Seu conteúdo físico reside no fato de que a gravidade é atrativa quando a condição de energia forte é satisfeita. O resultado desta atração é a focalização de geodésicas.

Sob as hipóteses do teorema da focalização geodésica, é claro que

$$
\frac{d \theta}{d \tau} \leq-\frac{\theta^{2}}{3}
$$

Portanto,

$$
\theta^{-1}(\tau) \geq \theta_{0}^{-1}+\frac{\tau}{3}
$$

onde $\theta_{0}=\theta(0)$. Ou seja, uma congruência inicialmente convergente $\left(\theta_{0}<0\right)$ implicará $\theta \rightarrow-\infty$ em um tempo próprio $\tau \leq 3 /\left|\theta_{0}\right|$. Isto quer dizer que a congruência desenvolverá uma cáustica, um ponto no qual uma coleção de geodésicas se encontram.

Embora uma singularidade na estrutura do espaço-tempo implique focalização geodésica, o contrário nem sempre é verdade. Para que seja provada a existência de singularidades do espaço-tempo, é necessário que, uma vez demonstrada a existência de cáusticas, seja inferida a existência de geodésicas de comprimento extremo. Um exemplo seria uma geodésica do tipo tempo que "termina" em um intervalo de tempo próprio finito. A existência de singularidade fica então condicionada à existência de geodésicas não extensíveis. A idéia básica, portanto, é que, se o espaço-tempo é não-singular, então todas as geodésicas são extensíveis ao longo de um intervalo infinito do parâmetro afim. 
No caso particular do universo homogêneo e isotrópico de FRW, basta o seguinte teorema básico de singularidade [82] para inferir a existência de um ponto singular em um instante de tempo passado finito:

Teorema: Em um universo onde $\rho+3 P \geq 0$ e $\omega^{\alpha \beta}=0$, para qualquer instante no qual $H_{0}=\theta_{0} / 3>0$, houve um tempo passado $t_{0}<H_{0}^{-1}$ tal que $a \rightarrow 0$ quando $t \rightarrow t_{0}$. Em $t_{0}$, ocorre uma singularidade do espaço-tempo, onde $\rho \rightarrow \infty$ e $P \rightarrow \infty$.

Portanto, as hipóteses do teorema implicam $(i)$ validade da condição de energia forte e $(i i)$ divergência cada vez mais lenta, na direção futura, de uma congruência ortogonal de hipersuperfície. 


\section{Capítulo 5}

\section{Ricochete}

Os teoremas de singularidade, ao mesmo tempo em que provam que singularidades não são apenas um artefato devido à presença de simetrias, nos indicam como seria possível encontrar soluções das equações de Einstein livres de singularidade.

Levando-se em conta as hipóteses assumidas nos teoremas de Hawking e Penrose, um caminho possível seria a violação da condição de energia forte. Este é o caso, por exemplo, de espaços-tempos inflacionários [2, 3, 4], nos quais a matéria é descrita por um campo escalar. Esta violação, no entanto, mostrou-se insuficiente: como provaram Borde, Guth e Vilenkin [6, 5, 7], independentemente do tensor de momento e energia satisfazer ou não condições de energia, espaços-tempos inflacionários são, necessariamente, geodesicamente incompletos para o passado.

Uma alternativa seria assumir que, ao invés do modelo do big bang, um outro cenário se impusesse, a saber, que, em um período anterior à ela, o universo se contraiu até um estado de energia e curvatura máximas (e finitas), depois do qual passou a se expandir. Resta saber que tipo de matéria poderia produzir um tal cenário, também conhecido por ricochete [73, 74, 83, 84, 72], no âmbito da relatividade geral. Tipicamente, se o universo é dominado por um fluido hidrodinâmico de matéria e radiação, a segunda equação de Friedmann proíbe a ocorrência de um ricochete, porque, nesse caso, temos $\rho+3 P \geq 0$, de tal modo que $\ddot{a}<0$, ou seja, não existe ponto de mínimo do fator de escala. Se, como no caso da inflação, a matéria for descrita por um campo escalar, de tal modo que $\ddot{a}>0$, então, pela primeira equação de Friedmann, o universo deve ser fechado $(k=+1)$, porque somente nesse caso é que podemos ter $H=\dot{a} / a=$ 
0, ou seja, o fator de escala atinge um valor extremo. Um mecanismo, portanto, deve existir para que, como em um universo inflacionário, a curvatura espacial $k$ possa ir a zero em um tempo suficientemente rápido. Um mecanismo desse tipo é, conforme veremos, possível [18]. No entanto, o problema agora é que não há maneira de se unir a fase pós-ricochete com as eras usuais do modelo cosmológico padrão [11, 21].

Outras possibilidades de se construir modelos de ricochete existem, porém fora do domínio da relatividade geral. Este é o caso, por exemplo, dos chamados modelos de pré-big bang $[14,85]$ inspirados em teoria de cordas, em que se supõe que, contrariamente ao modelo cosmológico padrão, o universo iniciou sua evolução do mais simples estado inicial possível concebido pela teoria de cordas, a saber, do vácuo perturbativo, no qual o universo seria praticamente (i) vazio, (ii) frio e ( iii) plano. A fase de pré-big bang (no quadro das cordas) corresponderia então a uma longa fase de super inflação $(\dot{H}>0)$, dominada por um campo escalar chamado de dilaton, que reaqueceria o universo, levando-o do estado acima descrito a um estado (i) denso, (ii) quente e (iii) altamente curvo. Este cenário pitoresco, no entanto, mostrou-se problemático: a fase pré-big bang que, graças à uma reparametrização do dilaton, também pode ser descrita como uma fase de contração, não pode ser conectada suavemente ao período posterior de expansão [19]. Em outras palavras, enquanto a fase de contração, dominada pelo dilaton, possui uma singularidade futura, o período posterior emerge de uma. Também, do ponto de vista fenomenológico, é bastante improvável que modelos de pré-big bang sejam capazes de acomodar espectros invariantes de escala.

Em modelos de ricochete uma forma de se obter um espectro de flutuações invariante de escala [86] é no âmbito da cosmologia quântica. Neste modelo, para garantir a invariância do espectro, é importante que as flutuações cruzem o horizonte durante a fase de contração dominada por matéria não-relativística.

Neste capítulo vamos ver de que forma o ricochete cosmológico resolve os problemas clássicos do modelo cosmológico padrão. Em seguida, estudaremos o caso mais simples de um ricochete clássico não-singular [11], descrito em termos de um campo escalar (canônico) em um universo homogêneo e isotrópico com seção espacial de curvatura positiva. Discutiremos também um modelo de ricochete em cosmologia quântica capaz de produzir um espectro invariante, além de discutir a fenomenologia de modelos de pré-big bang, sobretudo no que diz respeito à (não-)invariância do espectro de potência. 


\subsection{Solução dos problemas do modelo de FRW}

A suposição de que o universo evoluiu não de um ponto singular, mas de um ricochete, remete-nos naturalmente às questões de como poderíamos explicar o fato da radiação cósmica de fundo ser isotrópica em escalas de distância bem maiores que o horizonte e por que o universo é observado como sendo espacialmente plano. Vamos ver nessa seção como uma fase de ricochete pode resolver esses problemas.

Problema do horizonte: o horizonte de partículas, que é a distância percorrida pela luz desde um tempo inicial $t_{i}$ até um tempo $t>t_{i}$, é definido pela equação (2.28) como

$$
D_{H}(t) \equiv a(t) \int_{t_{i}}^{t} \frac{d t^{\prime}}{a\left(t^{\prime}\right)} .
$$

O problema que ocorre no modelo de FRW é que não somente a integral (5.1) é finita, mas também que o seu valor é bem menor que a escala de Hubble atual (ver equação (2.29)).

A solução de ricochete é similar à solução inflacionária, no sentido de que a integral (5.1) passa a divergir, com a diferença de que, neste caso, ela diverge graças ao fato de que em modelos de ricochete não existe um tempo inicial, ou seja, supomos que $t_{i} \rightarrow-\infty$.

Concretamente, consideremos um universo de seção espacial plana cuja dinâmica é induzida por um fluido perfeito com equação de estado constante $w=P / \rho$. Nesse caso, o fator de escala se comporta como

$$
a(t) \propto|t|^{\frac{2}{3(1+w)}}
$$

Vamos assumir por simplicidade que o ricochete ocorre em $t=0$, de tal modo que a fase de contração corresponde a $t<0$ e a fase de expansão a $t>0$. O horizonte de partículas é

$$
D_{H}(t)=\frac{3(1+w)}{1+3 w}\left\{\left|t_{i}\right|^{(1+3 w) /[3(1+w)]}-|t|^{2 /[3(1+w)]}+t\right\} .
$$

Logo, se $w>-1 / 3$, quando $t_{i} \rightarrow-\infty$ o horizonte de partículas diverge. Para todo $t$ antes ou depois do ricochete, o horizonte é infinito, e assim o será para qualquer instante posterior.

Problema da planura: este problema torna-se manifesto ao considerarmos a equação que determina a evolução temporal do parâmetro de densidade total 
$\Omega$, equação (2.30), dada por

$$
\frac{d}{d t}|\Omega-1|=-\frac{2 \ddot{a}}{\dot{a}^{3}}
$$

As observações indicam que $\Omega$ hoje é muito próximo da unidade, o que implica, por sua vez, curvatura espacial praticamente nula. Da equação (5.4), vemos que, em um universo em expansão $(\dot{a}>0)$ desacelerada $(\ddot{a}<0)$, $\Omega$ deve ter sido ainda mais próximo de 1 no passado, porque nesse caso $|\Omega-1|$ é uma função crescente do tempo, indicando um fino e improvável ajuste de condições iniciais. A inflação requer $\dot{a}>0$ e $\ddot{a}>0$, de tal modo que $|\Omega-1|$ decresce com o tempo. Portanto, no cenário inflacionário, é natural que $\Omega$ seja efetivamente próximo da unidade já na era da radiação. Em outras palavras, é natural que o universo primordial tenha curvatura espacial plana.

Esta, porém, não é a única possibilidade. Podemos, evidentemente, impor que $\dot{a}<0$ e $\ddot{a}<0$, ou seja, o universo se contrai desaceleradamente. Na fase subseqüente de expansão, $|\Omega-1|$ volta a crescer com o tempo ${ }^{1}$. Portanto, neste cenário, dizemos que o universo é plano hoje porque ele se contraiu mais do que se expandiu.

\subsection{Ricochete clássico}

Vamos discutir nesta seção um modelo de ricochete clássico apresentado em [11], não-singular, no qual a gravidade é descrita pela relatividade geral e o conteúdo de matéria por um campo escalar (canônico) em um universo aberto. O estudo desta classe de modelos nos ajuda a compreender a maneira como as perturbações escalares podem se propagar através do ricochete. Em particular, verifica-se que os modos dominante e sub-dominante da fase de contração se misturam na fase de expansão de uma maneira não-trivial, implicando alterações do espectro de perturbações escalares.

\subsubsection{Matriz de transferência}

Antes de discutirmos a dinâmica do ricochete, é útil esclarecermos o significado da mistura de modos.

\footnotetext{
${ }^{1}$ Estamos, naturalmente, assumindo que o conteúdo de matéria é do mesmo tipo em ambas as fases.
} 
Antes do ricochete, para um dado número de onda $k$, as perturbações são caracterizadas por dois modos: um modo dominante $D_{-}(k)$ e um modo subdominante $S_{-}(k)$, onde o rótulo de sinal de menos denota objetos calculados na fase de contração (pré-ricochete). Descrevemos o efeito do ricochete sobre $D_{-}(k)$ e $S_{-}(k)$ em termos da matriz de transferência $T(k)$ definida por

$$
\left(\begin{array}{c}
D_{+} \\
S_{+}
\end{array}\right)=\left(\begin{array}{ll}
T_{11} & T_{12} \\
T_{21} & T_{22}
\end{array}\right)\left(\begin{array}{c}
D_{-} \\
S_{-}
\end{array}\right)
$$

onde o rótulo de sinal de mais denota objetos calculados na fase de expansão (pós-ricochete). Portanto, vemos da equação matricial (5.5) que, em geral, para um dado $k$, o modo dominante $D_{+}$é uma combinação linear de $D_{-}$e $S_{-}$:

$$
D_{+}(k)=T_{11}(k) D_{-}(k)+T_{12}(k) S_{-}(k) .
$$

A equação (5.6) descreve várias situações: vamos supor que $D_{-}(k)$ carregue um espectro invariante de escala. Se $T_{11}(k)=0$ e $T_{12}(k) \neq 0$, a influência do ricochete sobre o modo $k$ se traduz em uma perda da invariância do espectro. Dizemos neste caso que ouve uma $k$-inversão de modos. Note que, ainda que $T_{11}(k) \neq 0$, a invariância do espectro pode não estar garantida dependendo da forma de $T_{11}(k)$. De modo análogo, se $D_{-}(k)$ carrega um espectro dependente de escala, dependendo da forma de $T_{11}(k)$, o espectro de $D_{+}(k)$ pode vir a se tornar invariante.

A esse respeito, o que se postula usualmente, notavelmente em modelos de pré-big bang [14], é que, ao menos para escalas de interesse astrofísico [14], o ricochete, por ter uma duração arbitrariamente curta (que é efetivamente o que ocorre em teorias de gravidade a derivadas mais altas, como constataremos [13]), não produz efeitos significativos sobre o espectro de flutuações. Tecnicamente, esse postulado implica uma matriz de transferência independente do número de onda. Logo, em cenários de pré-big bang, é suficiente encontrar situações em que a parte invariante da fase pré-ricochete seja transferida à parte dominante da fase pós-ricochete (sem $k$-inversão de modos). Uma conclusão de importância do trabalho [11] é que, mesmo em uma situação simples, a matriz de transferência é dependente de $k$. 


\subsubsection{Equações de movimento}

Em um espaço-tempo homogêneo e isotrópico, a métrica é dada pela métrica de Friedmann-Robertson-Walker, definida como

$$
d s^{2}=a^{2}(\eta)\left[-d \eta^{2}+\frac{d r^{2}}{1-K r^{2}}+r^{2}\left(d \theta^{2}+\sin ^{2} \theta d \phi^{2}\right)\right]
$$

$\eta$ é o tempo conforme, definido em termos do tempo comóvel como $d \eta=d t / a$. $K$ é a curvatura da seção espacial, normalizada a $\{-1,0,1\}$.

A matéria será descrita por um campo escalar homogêneo $\varphi(\eta)$ cuja densidade de energia e pressão são dadas por

$$
\begin{aligned}
\rho & =\frac{\varphi^{\prime 2}}{2 a^{2}}+V(\varphi) \\
P & =\frac{\varphi^{\prime 2}}{2 a^{2}}-V(\varphi)
\end{aligned}
$$

onde a linha denota derivação em relação ao tempo conforme e $V(\varphi)$ é o potencial do campo escalar.

As equações de Einstein que relacionam o fator de escala com a densidade e pressão do campo escalar são

$$
\begin{aligned}
\frac{3}{a^{2}}\left(\mathcal{H}^{2}+K\right) & =8 \pi G \rho \\
-\frac{1}{a^{2}}\left(2 \mathcal{H}^{\prime}+\mathcal{H}^{2}+K\right) & =8 \pi G P
\end{aligned}
$$

onde $\mathcal{H} \equiv a^{\prime} / a$.

Apesar de um ricochete clássico violar a condição de energia forte, para que seja possível ele deve ao mesmo tempo obedecer à condição de energia nula, pois

$$
(\rho+P)=\frac{\varphi^{\prime 2}}{a^{2}}>0 .
$$

No nível perturbativo a métrica (5.7) fica

$d s^{2}=a^{2}(\eta)\left\{-(1+2 \phi) d \eta^{2}+2 \partial_{i} B d \eta d x^{i}+\left[(1-2 \psi) \gamma_{i j}^{(3)}+2 \nabla_{i} \partial_{j} E\right] d x^{i} d x^{j}\right\}$

onde $\gamma_{i j}^{(3)}$ é a métrica das seções espaciais e $\nabla_{i}$ denota a derivada covariante compatível com $\gamma_{i j}^{(3)}$. As autofunções do operador de Laplace-Beltrami defini- 
das nas seções espaciais obedecem à equação

$$
\Delta f_{n}=-n(n+2) f_{n}
$$

onde $n$ é um número inteiro adimensional, uma vez que estamos considerando coordenadas $\{\eta, \boldsymbol{x}\}$ adimensionais, o que também significa que o operador $\Delta$ é ele mesmo adimensional. Os modos $n=0,1$ correspondem a modos de calibre [87], razão pela qual basta considerarmos modos tais que $n>1^{2} 3$.

As perturbações escalares são descritas pelas funções $\phi, B, \psi$ e $E$, a partir das quais se define os potenciais de Bardeen invariantes de calibre como [66]

$$
\Phi \equiv \phi+\frac{1}{a}\left[a\left(B-E^{\prime}\right)\right]^{\prime}, \Psi \equiv \psi-\mathcal{H}\left(B-E^{\prime}\right)
$$

Para fluidos de matéria sem tensão anisotrópica, como é o caso para campos escalares, tem-se $\Phi=\Psi$. Além disso, por causa do lema de Stewart [89], a forma dos potenciais de Bardeen são as mesmas independentemente do valor de $K$.

Para o setor da matéria, o campo escalar é escrito como $\varphi+\delta \varphi(\eta, \boldsymbol{x})$, onde $\delta \varphi(\eta, \boldsymbol{x})$ corresponde às flutuações não-homogêneas, que podem também ser descritas em termos da variável invariante de calibre $\delta \varphi^{(i c)} \equiv \delta \varphi+\varphi^{\prime}\left(B-E^{\prime}\right)$.

Das equações de Einstein invariantes de calibre de primeira ordem nos campos de flutuação se deduz a equação mestra para o potencial de Bardeen, dada por

$$
\Phi^{\prime \prime}+2\left(\mathcal{H}-\frac{\varphi^{\prime \prime}}{\varphi^{\prime}}\right) \Phi^{\prime}+\left[n(n+2)+2\left(\mathcal{H}^{\prime}-\mathcal{H} \frac{\varphi^{\prime \prime}}{\varphi^{\prime}}-2 K\right)\right] \Phi=0 .
$$

Introduzindo uma nova variável $u$ através de

$$
\Phi \equiv 4 \pi G(\rho+P)^{1 / 2} u=\sqrt{6 \pi G} \frac{\mathcal{H}}{a^{2} \theta} u
$$

\footnotetext{
${ }^{2} \mathrm{Na}$ referência [87], os autovalores correspondentes à equação de Helmholtz $\Delta \Psi_{k}=k^{2} \Psi_{k}$ são definidos em termos da nova variável $n$ por $k^{2}+K=(n+K)^{2}$, ou seja, $k^{2}=n(n+2 K)+$ $K(K-1)$. Quando $K=+1$, então $k^{2}=n(n+2)$, como na equação (5.14).

${ }^{3}$ Para escalas de distância de interesse astrofísico, que são aquelas situadas no intervalo $\left[10^{-2} h^{-1} \mathrm{Mpc}, 10^{3} h^{-1} \mathrm{Mpc}\right]$, os valores de $n$ correspondentes se situam entre 6 e $6 \times 10^{6}$ para um parâmetro de densidade total $\Omega_{0} \simeq 1,01$. Ver, por exemplo, referência [88].
} 
onde a função $\theta$ é definida como

$$
\theta=\frac{1}{a}\left(\frac{\rho}{\rho+P}\right)^{1 / 2}\left(1-\frac{3 K}{8 \pi G \rho a^{2}}\right)^{1 / 2}
$$

a equação mestra pode ser escrita como

$$
\begin{gathered}
u^{\prime \prime}+\left[n(n+2)-\frac{\theta^{\prime \prime}}{\theta}-3 K\left(1-c_{s}^{2}\right)\right] u=0, \\
c_{S} \equiv \frac{P^{\prime}}{\rho^{\prime}}=-\frac{1}{3}\left(1+2 \frac{\varphi^{\prime \prime}}{\mathcal{H} \varphi^{\prime}}\right) .
\end{gathered}
$$

A última igualdade de (5.20) segue imediatamente da Equação de KleinGordon

$$
\varphi^{\prime \prime}+2 \mathcal{H} \varphi^{\prime}+a^{2} \frac{d V}{d \varphi}=0
$$

A equação para a variável $u$ tem a forma de uma equação de Schrödinger independente do tempo. No caso em que $K \neq 0$, no entanto, o termo de potencial efetivo não pode ser escrito como a segunda derivada de alguma função ( $\theta$ quando $K=0)$ por causa da presença de $3 K\left(1-c_{s}^{2}\right)$ no termo de freqüência. Portanto, em escalas super-Hubble, quando o termo $n(n+2)$ torna-se desprezível, contrariamente ao caso plano, as soluções não mais serão dadas por $u=\theta$ e $u=\theta \int \theta^{-2} d \eta^{\prime}$.

\subsubsection{Descrição do ricochete}

Vamos assumir que o universo passa por uma transição regular entre fases de contração e expansão em $\eta=0$. Uma descrição simples é o ricochete de de Sitter, em que o fator de escala é $a(t)=a_{0} \cosh \left(t / a_{0}\right)$, em termos do tempo comóvel, e $a(\eta)=a_{0} \sqrt{1+\tan ^{2}\left(\eta / \eta_{0}\right)}$, em termos do tempo conforme. Identificamos $a_{0}$ como sendo o raio do universo no ricochete e $\eta_{0}$ como o tempo de duração conforme do ricochete. Uma propriedade remarcável deste modelo é que o potencial efetivo de $u$ não depende de $\eta$, ou seja,

$$
V_{u}^{(d S)}=4-\frac{1}{\eta_{0}^{2}}
$$

Uma conseqüência importante emerge do potencial (5.22): seu valor máximo é $V_{u}^{(d S)}=3$, quando $\left|\eta_{0}\right|=1$. Logo, o potencial só interage com modos tais que $n=0,1$, que correspondem a modos de calibre [87]. 
Portanto, para compreendermos a maneira como as flutuações se propagam pelo ricochete, devemos considerar modelos mais gerais.

A regularidade deste tipo de transição implica, em particular, que o fator de escala e o campo escalar são analíticos em $\eta=0$, ou seja, que admitem uma expansão de Taylor em torno de $\eta=0$, isto é

$$
\begin{gathered}
\varphi(\eta)=\varphi_{0}+\varphi_{0}^{\prime} \eta+\frac{\varphi_{0}^{\prime \prime} \eta^{2}}{2}+\mathcal{O}\left(\eta^{3}\right) \\
a(\eta)=a_{0}\left[1+\frac{1}{2}\left(\frac{\eta}{\eta_{0}}\right)^{2}+\delta\left(\frac{\eta}{\eta_{0}}\right)^{3}+\frac{5}{24}(1+\xi)\left(\frac{\eta}{\eta_{0}}\right)^{4}\right] .
\end{gathered}
$$

Além dos parâmetros $a_{0}$ e $\eta_{0}$ definidos no último parágrafo, há dois parâmetros $\delta$ e $\xi$ que controlam a magnitude dos termos de terceira e quarta ordens. Expressam, além disso, o desvio em relação a um ricochete de de Sitter. De fato, expandindo $a(\eta)=a_{0} \sqrt{1+\tan ^{2}\left(\eta / \eta_{0}\right)}$ em torno da origem, obtemos exatamente a expressão (5.24) $\operatorname{com} \delta=0=\xi$.

Uma importante conclusão que segue deste modelo generalizado é que o tempo de duração do ricochete deve ser tal que $\eta_{0}>1$, ou seja, o ricochete tem uma duração mínima, não-arbitrária e diferente de zero. Caso contrário, teríamos $\varphi^{\prime 2}<0$, o que é impossível. Para vermos isso, é necessário encontrar as relações existentes entre os coeficientes $a_{0}, \delta$ e $\xi$ da expansão (5.24) e os valores do campo e de sua primeira derivada em $\eta=0$. Concretamente, substituímos as expansões (5.23) e (5.24) nas equações (5.10) e (5.11). Em ordem dominante, tem-se

$$
a_{0}^{2}=\frac{6-8 \pi G \varphi_{0}^{\prime 2}}{16 \pi G V\left(\varphi_{0}\right)}, \Upsilon=4 \pi G \varphi_{0}^{\prime 2}
$$

onde $\Upsilon \equiv 1-1 / \eta_{0}$. Portanto, para que a condição de energia nula (5.12) seja satisfeita, devemos ter $\eta_{0}>1$.

A condição sobre a duração do ricochete torna-se mais restritiva ao considerarmos a influência desta transição sobre a evolução da variável $u$. De fato, para que a influência do ricochete sobre as perturbações de interesse (que correspondem aos modos com $n \gg 1[14,87]$ ) seja efetiva, o tempo do ricochete deve ser tal que $\eta_{0} \gtrsim 1$. Isto torna-se claro pela expressão para o potencial 
efetivo $V_{u}$, dado neste caso por

$$
V_{u}=\frac{2-10 \eta_{0}^{2}+8 \eta_{0}^{4}+5 \xi}{2 \eta_{0}^{2}\left(\eta_{0}^{2}-1\right)}
$$

A propriedade relevante do potencial (5.27) é que ele diverge no limite $\eta_{0} \rightarrow 1$. Como as escalas de relevância astrofísica são tais que $n \gg 1$, então a condição necessária para que o ricochete altere o espectro de flutuações é que $\eta_{0}$ seja próximo da unidade ou, posto de outra forma, que o parâmetro $\Upsilon$ seja muito menor que 1.

\subsubsection{Modificação do espectro de flutuações}

De acordo com o que discutimos na última subseção, é então suficiente estudarmos a influência do ricochete sobre as flutuações, codificada na matriz de transferência, no limite $\Upsilon \rightarrow 0$. Nesse limite, o potencial efetivo de $u$ pode ser aproximado para

$$
V_{u}=-C_{\Upsilon} \Delta_{\Upsilon}(\eta)
$$

onde a constante $C_{\Upsilon}$ é dada por $C_{\Upsilon} \equiv \sqrt{-5 \pi^{2} \xi /(8 \Upsilon)}$ e $\Delta_{\Upsilon}(\eta)$ é uma representação da distribuição de Dirac, isto é

$$
\lim _{\Upsilon \rightarrow 0} \Delta_{\Upsilon}=\delta^{(1)}(\eta)
$$

A equação mestra (5.19) fica então

$$
u^{\prime \prime}+\left[n(n+2)+C_{\Upsilon} \delta^{(1)}(\eta)\right] u=0,
$$

uma equação conhecida no contexto da mecânica quântica. As condições de compatibilidade são $[u]=0$ e $\left[u^{\prime}\right]=-C_{\Upsilon} u(0)$, a última seguindo de uma integração da equação (5.28) através de um intervalo infinitesimal em torno de $\eta=0$.

Nas regiões onde o potencial pode ser desprezado, isto é, antes e depois do ricochete, a solução para $u$ é

$$
u_{i}(n, \eta)=A_{i}(n) f_{i}(n, \eta)+B_{i}(n) g_{i}(n, \eta), i=\mathrm{I}, \mathrm{II}
$$


onde as funções dependentes de $\eta$ são

$$
f_{\mathrm{I}, \mathrm{II}}=\frac{\mathrm{e}^{-i \sqrt{n(n+2)} \eta}}{\sqrt{2 \sqrt{n(n+2)}}}, g_{\mathrm{I}, \mathrm{II}}=\frac{\mathrm{e}^{i \sqrt{n(n+2)} \eta}}{\sqrt{2 \sqrt{n(n+2)}}}
$$

A matriz de transferência relacionando as amplitudes das fases pré-ricochete e pós-ricochete é, portanto,

$$
T(n)=-i \sqrt{\frac{-5 \pi^{2} \xi}{32 n(n+2)}} \frac{1}{\Upsilon^{1 / 2}}\left(\begin{array}{cc}
1 & 1 \\
-1 & -1
\end{array}\right),
$$

mostrando que, mesmo neste modelo simples de um ricochete clássico com um campo escalar em um universo aberto, os modos são misturados e o espectro modificado.

\subsection{Cosmologia quântica}

Vamos nesta seção discutir um modelo de ricochete [86] capaz de acomodar um espectro de perturbações invariante de escala.

No procedimento usual, a quantização ocorre no nível perturbativo, enquanto que o espaço-tempo de fundo permanece clássico. Um exemplo típico é a quantização de perturbações cosmológicas sobre um espaço-tempo inflacionário. No trabalho [86], a quantização vai além da abordagem usual ao quantizar também o espaço-tempo de fundo.

A ação considerada é dada pela ação de Einstein-Hilbert mais um termo de fonte que corresponde a um fluido perfeito com equação de estado constante 4 :

$$
S=-\int d^{4} x \sqrt{-g}\left(\frac{R}{6 \ell_{P l}^{2}}+\rho\right),
$$

onde $R$ é o escalar de Ricci, $\ell_{P l}^{2} \equiv 8 \pi G / 3$ é o comprimento de Planck em unidades naturais $(\hbar=1=c)$ e $\rho$ é a densidade de energia do fluido cuja pressão $P$ é dada pela relação $P=w \rho$, onde $w$ é uma constante não-nula.

A métrica $g_{\mu \nu}$ é definida como

$$
g_{\mu \nu}=g_{\mu \nu}^{(0)}+h_{\mu \nu},
$$

\footnotetext{
${ }^{4}$ Vamos adotar, nesta seção, a notação original de [86].
} 
onde $g_{\mu \nu}^{(0)}$ é a métrica de um espaço-tempo homogêneo e isotrópico tal que

$$
d s^{2} \equiv g_{\mu \nu}^{(0)} d x^{\nu} d x^{\mu}=N^{2}(\tau)-a^{2}(\tau) \delta_{i j} d x^{i} d x^{j}
$$

e $h_{\mu \nu}$ corresponde a perturbações escalares em torno de $g_{\mu \nu}^{(0)}$, definidas como

$$
h_{00}=2 N^{2} \phi, h_{0 i}=-N a B_{, i}, h_{i j}=2 a^{2}\left(\psi \delta_{i j}-E_{, i j}\right)
$$

A função lapso pode ser escolhida como $N=a^{3 w}$, de tal modo que $\tau$ pode ser identificado como o tempo cósmico se o fluido for constituído por poeira, ou como sendo o tempo conforme para fluidos de radiação.

A Hamiltoniana de segunda ordem é então obtida por substituição das relações (5.34) e (5.35) na ação de Einstein-Hilbert (5.32). Sua forma simplificada foi obtida em [90]. Aqui, vamos apenas reproduzir o resultado:

$$
H=N\left[H_{0}^{(0)}+H_{0}^{(2)}\right]+\Lambda_{N} P_{N}+\int d^{3} x \phi \pi_{\psi}+\int d^{3} x \Lambda_{\phi} \pi_{\phi},
$$

onde

$$
\begin{gathered}
H_{0}^{(0)} \equiv-\frac{\ell_{P l}^{2} P_{a}^{2}}{4 a V}+\frac{P_{\tau}}{a^{3 w}} \mathrm{e} \\
H_{0}^{(2)} \equiv \frac{1}{2 a^{3}} \int d^{3} x \pi^{2}+\frac{a w}{2} \int d^{3} x v^{, i} v_{, i} .
\end{gathered}
$$

Na equação (5.37), $V$ é o volume comóvel das hiper-superfícies do tipo espaço do espaço-tempo de fundo. As quantidades $N, \Lambda_{N}, \phi$ e $\Lambda_{\phi}$ desempenham o papel de multiplicadores de Lagrange dos vínculos $H_{0}^{(0)}+H_{0}^{(2)} \approx 0, P_{N} \approx 0$, $\pi_{\psi} \approx 0$ e $\pi_{\phi} \approx 0$, respectivamente. Os momentos $P_{a}, \pi_{\psi}, \pi_{\phi}, \pi, P_{N}$ e $P_{\tau}$ são conjugados a $a, \psi, \phi, v, N$ e $\tau$, respectivamente. A variável $v$ é relacionada ao potencial de Bardeen $\Phi$ por

$$
\Phi_{, i}^{, i}=-\frac{3 \ell_{P l}^{2} \sqrt{(w+1) \rho_{0}}}{2 \sqrt{w}} a\left(\frac{v}{a}\right)^{\prime}
$$

O vínculo $H_{0}^{(0)}+H_{0}^{(2)}$ é o vínculo responsável pela dinâmica, do qual decorrem as equações de Einstein de ordens 0 e 1 nas perturbações. Os outros vínculos, por suas vezes, implicam que $N, \psi$ e $\phi$ não são relevantes. O único grau de liberdade dinâmico perturbativo é $v$.

O próximo passo é a quantização tanto do fundo como das perturbações escalares pelo método de Dirac. O resultado deste procedimento [90] é que o 
funcional de onda $\Psi$ depende apenas de $a, v$ e $\tau$. A interpretação de Bohm-de Broglie [91] é equivalente a assumir que $\Psi$ pode ser fatorado como

$$
\Psi[a, v, \tau]=\Psi^{(0)}[a, \tau] \times \Psi^{(2)}[v, \tau]
$$

onde a função $a(\tau)$, obtida da equação de ordem 0 para $\Psi$ (equação de Wheelerde Witt), não mais é a solução clássica das equações de fundo, mas uma trajetória bohmiana do espaço-tempo de fundo quantizado, cuja forma explícita é [86]

$$
a(\tau)=a_{0}\left[1+\left(\frac{\tau}{T_{0}}\right)^{2}\right]^{1 /[3(1-w)]},
$$

onde $a_{0}$, o valor do fator de escala no ricochete, e $T_{0}$ são constantes arbitrárias a serem eventualmente determinadas pelas observações, e onde o parâmetro $\tau$ está relacionado com o tempo conforme por

$$
d \eta=[a(\tau)]^{3 w-1} d \tau
$$

A solução (5.41) é bem-comportada é tende à solução clássica quando $\tau \rightarrow$ $\pm \infty$.

A equação para $v$ no espaço de Fourier é obtida da equação de segunda ordem para $\Psi[a, v, \tau]$, e é dada por [90]

$$
v_{k}^{\prime \prime}+\left(w k^{2}-\frac{a^{\prime \prime}}{a}\right) v_{k}=0
$$

onde $v$ se reduz à variável de Mukhanov-Sasaki quando o fundo satisfaz à equação clássica de Einstein, e onde a "linha" denota derivada com respeito ao tempo conforme $\eta$. O potencial $V \equiv a^{\prime \prime} / a$ se comporta, qualitativamente, como o potencial para as flutuações do caso inflacionário: ele é desprezível quando $|\eta| \rightarrow \infty$, ou seja, longe do ricochete, e tem o seu máximo em torno de $\eta=0$. Dois exemplos de interesse são os seguintes: para um fluido de radiação,

$$
V_{\text {rad }}=\frac{1}{T_{0}^{2}\left(1+\frac{\eta^{2}}{T_{0}^{2}}\right)^{2}},
$$


e para um fluido de poeira,

$$
V_{\text {poeira }}=\frac{2 a_{0}^{2}}{9 T_{0}^{2}} \frac{3+x^{2}}{\left(1+x^{2}\right)^{4 / 3}},
$$

onde $x \equiv \tau / T_{0}$. Em ambos os casos, o potencial vai a zero no limite $|\tau| \rightarrow \infty$, e atinge um máximo em $\eta=0$. No infinito passado e no infinito futuro, quando $k^{2} \gg V$, a escala das perturbações é bem menor que a escala de curvatura. Nessas regiões, as perturbações oscilam, e podem, portanto, ser definidas no infinito passado como estando em um estado quântico de vácuo. Perto do ricochete, as escalas das flutuações tornam-se maiores que a escala de curvatura e eventualmente acabam penetrando no potencial $\left(k^{2} \ll V\right)$, quando são então amplificadas. Finalmente, no infinito futuro, voltam a ser bem menores e oscilam novamente, mas desta vez com a amplitude amplificada.

Nesta classe de modelos o índice espectral $n_{S}$ é calculado como sendo [86]

$$
n_{S}=1+\frac{12 w}{1+3 w}
$$

Portanto, no limite $w \rightarrow 0$, o espectro será invariante de escala ${ }^{5}$, em concordância com as observações.

Utilizando a normalização do WMAP para a amplitude do espectro, que é da ordem de $10^{-10}$ [1], é possível determinar os dois parâmetros desconhecidos $a_{0}$ e $T_{0}$ :

$$
T_{0} a_{0}^{3 w} \sim 10^{3} \ell_{P l}
$$

que corresponde a uma escala de comprimento para a qual correções quânticas são importantes, porém não suficientemente efetivas ao ponto de invalidar a equação de Wheeler-de Witt [86].

Embora o modelo descrito nesta seção seja capaz de acomodar um espectro invariante de escala, uma previsão envolvendo um número improvável emerge neste contexto: o valor mínimo para o fator de escala é extremamente grande: $a_{0} \gtrsim 10^{20} \ell_{P l}$. Felizmente, soluções generalizadas para o espaço-tempo de fundo [92] podem oferecer um mecanismo capaz de resolver problemas desta natureza.

\footnotetext{
${ }^{5}$ Embora ligeiramente azul, fato este que também não está excluído pelas observações [1]. Um outro teste que poderia eventualmente diferenciar entre modelos inflacionários de modelos de ricochete é através da relação de consistência, ou a razão entre as amplitudes do espectro escalar e tensorial. Enquanto modelos inflacionários prevêem uma relação linear em $n_{S}-1$, em modelos de ricochete em cosmologia quântica [86] esta relação é proporcional a $\sqrt{n_{S}-1}$.
} 


\subsection{Modelos pré-big bang}

Teorias de gravidade efetivas quadri-dimensionais podem ser obtidas através da compactificação de dimensões extras de alguma teoria fundamental (teoria M ou teoria de cordas, por exemplo). Um exemplo dessa classe é o cenário de pré-big bang [14], baseado na ação efetiva de baixas energias da teoria de cordas. Nesta descrição do universo encontramos dois ramos, o primeiro dos quais corresponde a uma fase de superinflação e o segundo a uma fase de Friedmann na qual a curvatura é decrescente com o tempo. A união dos dois ramos é problemática [19]. Para que a transição ocorra suavemente, é necessário modificar a ação original com a introdução de termos de correção a derivadas mais altas [93, 94, 25, 95]. Ao transfomar a ação do quadro das cordas (ou quadro de Jordan) para o quadro de Einstein, o universo exibe uma contração na fase pré-big bang, na qual o fator de escala se comporta como $a \propto(-t)^{1 / 3}$. Nesse sentido o cenário de pré-big bang pode ser visto (no quadro de Einstein) como um cenário de ricochete cosmológico.

Vamos começar escrevendo a ação efetiva no quadro das cordas antes de transformá-la para o quadro de Einstein:

$$
S_{C}=\int d^{4} x \sqrt{-g} e^{-\phi}\left[\frac{1}{2} R+\frac{1}{2}(\nabla \phi)^{2}-V_{C}(\phi)\right],
$$

onde $\phi$ é o campo escalar dilaton com um potencial $V_{C}(\phi)$. O dilaton evolui de um regime fracamente acoplado $\left(e^{\phi} \ll 1, \phi<0, V_{C} \rightarrow 0\right)$, a um regime de acoplamento forte durante o qual o parâmetro de Hubble cresce (superinflação). Este ramo, que corresponde à fase de pré-big bang, se conecta com a fase de Friedmann subseqüente (se a singularidade entre elas for removida). Nesta transição, o parâmetro de Hubble atinge um valor máximo.

Fazendo uma transformação conforme

$$
\hat{g}_{\mu \nu}=e^{-\phi} g_{\mu \nu}
$$

a ação (5.48), no quadro de Einstein, fica

$$
S_{E}=\int d^{4} x \sqrt{-g}\left[\frac{1}{2} R-\frac{1}{4}(\hat{\nabla} \phi)^{2}-V_{E}(\phi)\right],
$$

onde $V_{E}(\phi) \equiv e^{\phi} V_{C}(\phi)$. Por uma redefinição de campo $\varphi= \pm \phi / \sqrt{2}$, a ação 
(5.50) fica

$$
S_{E}=\int d^{4} x \sqrt{-\hat{g}}\left[\frac{1}{2} \hat{R}-\frac{1}{2}(\hat{\nabla} \varphi)^{2}-V_{E}(\phi(\varphi))\right]
$$

Em um cenário típico [96], o potencial $V_{E}$ é dado por

$$
V_{E}=-V_{0} \exp \left(-\sqrt{\frac{2}{p}} \varphi\right)
$$

onde $0<p<1$. Conforme fora demonstrado na referência [96], a ação (5.51) é equivalente à ação de cenários "ekpiróticos" [16, 17], mas desta vez com $0<p \ll 1$. Em um cenário de pré-big bang, o dilaton inicia sua evolução em um regime fracamente acoplado com $\phi$ crescendo de $-\infty$. Logo, para que o potencial do dilaton seja importante em $\phi \rightarrow 0$ e desprezível em $\phi \rightarrow-\infty$, é necessário ter $\varphi=-\phi / \sqrt{2}$.

Em um espaço-tempo de fundo de FRW, o sistema com o potencial (5.52) admite a seguinte solução exata [96, 97]:

$$
a_{E} \propto\left|t_{E}\right|^{p}, H_{E}=\frac{p}{t_{E}}, V_{E}=-\frac{p(1-3 p)}{t_{E}^{2}}, \dot{\varphi}=\frac{\sqrt{2 p}}{t_{E}}
$$

A solução para $t_{E}<0$ descreve um universo em contração. Em cenários de pré-big bang, $p=1 / 3$, de tal modo que o potencial do dilaton se anula, ao passo que, para cenários "ekpiróticos", $0<p \ll 1$ [96]. No quadro de cordas as soluções (5.53) ficam [96]

$$
a_{C} \propto\left(-t_{S}\right)^{-\sqrt{p}}, \phi=-\frac{2 \sqrt{p}}{1-\sqrt{p}} \ln \left[-(1-\sqrt{p}) t_{C}\right]
$$

onde fica claro o comportamento crescente do dilaton na era super-inflacionária.

Com os resultados (5.53) podemos calcular o espectro gerado na fase de contração. Nesse caso, $\epsilon=1 / p$ e $\nu^{2}=[(3 p-1)(1-p)]^{2}$ nas equações $(3.41)$. Logo, segue da relação $n_{S}-1=3-2 \nu$ que o índice espectral das perturbações escalares é [98, 99, 100, 101, 102]

$$
n_{S}-1=\left\{\begin{array}{cl}
\frac{2}{1-p} & , 0<p \leq 1 / 3 \\
\frac{4-6 p}{1-p} & , 1 / 3 \leq p<1
\end{array}\right.
$$

Vemos então que, quando $p=1 / 3, n_{S}=4$, e quando $0<p \ll 1, n_{S} \simeq 3$, ou 
seja, em ambos os casos, o espectro é altamente inclinado para o azul.

Com o intuito de se calcular o espectro em um instante de tempo posterior, durante a fase de expansão, é preciso conectar a fase de contração com a fase posterior de Friedmann. Esta transição é realizada suavemente uma vez que termos de correção a derivadas mais altas são introduzidos na ação (5.48). Desta forma, é possível estudar a propagação de perturbações cosmológicas em um espaço-tempo de fundo regular sem utilizar métodos de "colagem" ("matching") de soluções. Os efeitos dos termos de derivadas mais altas na ação (5.48) sobre a evolução de perturbações em modelos de pré-big bang foram estudados numericamente por Cartier et al. [95] e por Tsujikawa et al. [103]. O resultado é, novamente, um espectro altamente inclinado para o azul, com $n_{S} \simeq 4$. No contexto de modelos "ekpiróticos" regulares com $0<p \ll$ 1, o índice espectral, calculado numericamente em [103], correspondendo ao espectro de perturbações escalares em um instante bem posterior ao ricochete é $n_{S} \simeq 3$. Os resultados iguais antes e depois do ricochete se devem ao fato de que, conforme demonstrado por Tsujikawa et al. [103], perturbações comóveis de curvatura são conservadas em escalas cosmológicas muito maiores que o raio de Hubble em uma vizinhança do ricochete.

\subsection{Comentários}

Se em um cenário simples de um espaço-tempo inflacionário dominado por um único campo escalar o espectro de perturbações invariante de escala aparece de forma natural, em cenários de ricochetes cosmológicos a situação é mais complicada. Como acabamos de ver, ricochetes em modelos de pré-big bang $[14,85]$ são mal definidos devido à existência de uma singularidade precisamente no ponto de mínimo do fator de escala [19]. Uma forma de curar esta patologia é introduzindo termos de correção de derivadas mais altas na métrica[93, 94, 25, 95, 103]. Entretanto, o espectro produzido por cenários deste tipo é altamente inclinado para o azul, ou seja, a potência é maior quanto maior for a freqüência [95, 104]. Um caminho possível para se obter um espectro aproximadamente invariante de escala é no contexto da cosmologia quântica, no qual as flutuações quânticas de vácuo cruzam o horizonte de Hubble durante a fase de contração dominada pela matéria [98, 105, 86, 18]. Nesses casos o espectro é ligeiramente inclinado para o azul, contrariamente ao o que prevêem modelos inflacionários com um único campo. As últimas obser- 
vações do WMAP [1] não favorecem essa previsão em particular, e tampouco a excluem, especialmente para pequenas inclinações do espectro [106].

Uma questão importante em relação aos modelos de cosmologia quântica diz respeito ao tipo de fluido presente no universo. O fato das flutuações cruzarem o horizonte de Hubble na fase dominada pela matéria não implica um universo dominado por um fluido único, isto é, um fluido puro de matéria. À medida que o universo se contrai, as densidades, assim como a temperatura do fluido, aumentam consideravelmente, atingindo eventualmente o ponto em que as massas das partículas se tornam desprezíveis. Em outras palavras, devemos esperar uma transição entre fases de matéria e radiação. O efeito desta transição sobre as perturbações da métrica é apenas o de alterar as suas amplitudes [65], sem para tanto modificar os espectros dos modos crescente e constante que caracterizam essas perturbações. O efeito do ricochete, por sua vez, é essencialmente o de misturar esses coeficientes, de tal maneira que o modo constante na fase pós-ricochete adquirirá a parte invariante do espectro da fase anterior de contração [107, 21]. Este fato é independente do tipo de fluido dominante [86]. Portanto, contanto que as flutuações cruzem o horizonte durante a era (de contração) da matéria, é de se esperar que o espectro produzido seja invariante de escala [18].

Outros modelos de ricochete compatíveis com um espectro invariante de escala são possíveis, notavelmente no contexto da relatividade geral com fluidos clássicos, mas com um dos fluidos tendo energia negativa [108, 109]. O espectro produzido será invariante de escala desde que o fluido de energia positiva, dominante no infinito passado, tenha uma equação de estado tal que $w \approx 0$. Além deles, há também modelos com um campo escalar clássico acoplado com a gravidade [105], cuja equação de estado também corresponde à matéria fria. Nesse caso, se a fase de contração puder ser conectada suavemente ao universo de FRW por um ricochete não-singular, a invariância do espectro será preservada.

Portanto, assim como universos inflacionários, universos eternos contendo fases de contração e expansão resolvem os problemas do horizonte e da planura e produzem um espectro de flutuações invariante de escala. São, além disso e contrariamente ao universo inflacionário, livres da singularidade inicial, desde, é claro, que o ricochete ele mesmo seja bem comportado. Um outro aspecto de importância que diferencia as duas classes de universos diz respeito à determinação das condições iniciais das flutuações. No caso infla- 
cionário, é conhecido o fato de que, se a inflação durou um tempo superior aos 60 "e-foldings" necessários para resolver os problemas do horizonte e da planura, então os comprimentos de onda de todas as escalas observadas hoje eram necessariamente menores que o comprimento de Planck no início da inflação [71]. Esta situação, na qual condições iniciais são fixadas em escalas sub-planckianas, é conhecida como problema transplanckiano [71]. Assim, podemos dizer que, de uma certa maneira, a invariância do espectro depende de condições iniciais fixadas em uma "zona de ignorância" onde as equações de Einstein deixam de ser válidas. De fato, é possível construir modelos simplificados baseados em física transplackiana que levam a correções significativas das previsões da cosmologia inflacionária [71].

Em universos eternos, o que se assume é que, no infinito passado, o universo era extremamente grande e diluído, maior do que qualquer escala observável hoje [18]. Isto significa que, para uma certa escala de interesse, houve um tempo suficientemente anterior ao ricochete durante o qual o seu comprimento de onda era bem menor do que a escala de curvatura, justificando o tratamento de perturbações como flutuações sobre um fundo de Minkowski.

Temos então o seguinte conjunto de fatos: universos inflacionários e universos eternos são ambos capazes de explicar as questões de por que o universo observável é plano e homogêneo, além de preverem um espectro de perturbações primordiais aproximadamente invariante de escala, embora no caso inflacionário isto seja realizado de uma maneira mais simples, com um único campo (escalar), em comparação com os ricochetes, que exigem seja a presença de fluidos com energia negativa, seja a introdução de uma teoria não clássica capaz de descrever um ricochete livre de singularidades. Por outro lado, o preço a se pagar pela simplicidade inflacionária é a introdução de $(i)$ um campo escalar, campo este jamais observado na natureza (pelo menos por enquanto) e ( $i i)$ de perturbações que vivem em escalas planckianas para as quais não há física conhecida capaz de descrevê-las corretamente.

Vemos então que, em ambos os casos, a descrição do universo primordial depende fundamentalmente de uma nova física, lacuna esta que, provavelmente, será preenchida pela teoria quântica da gravidade. Até o presente momento, em particular no que seguirá no próximo capítulo, vamos investigar ricochetes cosmológicos em uma teoria efetiva a derivadas mais altas [13]. Para simplificar este cenário já suficientemente complicado, vamos assumir que o universo é plano e preenchido por um fluido ordinário de matéria e radiação. Vere- 
mos que, pelo menos para certas condições iniciais, um universo em contração evolui por um ricochete não singular e se conecta suavemente ao universo de FRW, terminando em uma fase de expansão de de Sitter. 


\section{Capítulo 6}

\section{Gravidade generalizada}

Neste capítulo vamos iniciar o estudo de modelos cosmológicos em teorias de gravidade modificada. Conforme já comentamos em partes anteriores, o problema da energia escura, que também pode ser entendido como um fenômeno puramente gravitacional, sugere modificações da gravidade no regime infravermelho, ao passo que o problema da singularidade inicial aponta para a invalidade das equações de Einstein em regimes de alta curvatura.

Nesse sentido os modelos mais populares de energia escura são os modelos descritos pelas chamadas teorias $f(R)$, nas quais a Lagrangeana de EinsteinHilbert é generalizada por uma função arbitrária do escalar de Ricci $R$. Já no âmbito de modelos que tratam o universo primordial, podemos considerar extensões de alguma teoria fundamental como, por exemplo, a teoria de cordas ou a teoria M. É o caso, por exemplo, dos cenários de pré-big bang, nos quais a dualidade do fator de escala mapeia uma fase de contração (no quadro de Einstein) dominada pelo dilaton a uma fase de expansão que corresponde ao modelo usual de FRW.

Outra possibilidade é considerar teorias efetivas de ordem mais alta nos invariantes de curvatura [13]. Correções desse tipo são, de fato, naturais, seja pelo fato de que em regimes de alta curvatura termos de ordem mais alta tornam-se importantes, seja pelo fato de que teorias quadráticas em quatro dimensões são renormalizáveis, embora apresentem problemas de estabilidade, como discutiremos a seguir.

À parte os sucessos do modelo cosmológico padrão, restam ainda as questões de como explicar as naturezas da energia e matéria escuras. Possíveis candidatos a esta última decorrem de diferentes modelos teóricos, notavel- 
mente de teorias supersimétricas em que diferentes classes de neutralinos aparecem de modo natural [110], e cuja viabilidade tem sido testada com sucesso em simulações de formação de estruturas [111]. A energia escura, por sua vez, pode ser entendida em termos de uma pequena constante cosmológica $\Lambda \approx 3 \times 10^{-122} c^{3} / \hbar G$ ou também como um campo escalar, chamado de quintessência, em regime de rolamento lento na presença de um potencial [112, 113, 114]. Estas possibilidades, porém, não abordam a questão de por que a constante cosmológica é tão pequena e por que as densidades de matéria e energia escuras são hoje da mesma ordem de grandeza.

Para evitar a hipótese da matéria escura, Milgrom [115] propôs em 1983 uma modificação fenomenológica que explica com sucesso a curva de rotação de galáxias [116] e que recobra automaticamente a lei de Tully-Fisher $v_{\infty}^{4} \propto M_{g}$ [117], onde $M_{g}$ é a massa da galáxia e $v_{\infty}$ é a velocidade circular assintótica da matéria visível localizada na região exterior da galáxia. Neste cenário, a aceleração a de um corpo assume seu valor newtoniano $a_{N}=G M / r^{2}$ quando $a>a_{0}$, onde $a_{0} \approx 1,2 \times 10^{-10} \mathrm{~ms}^{2}$ é uma constante universal, enquanto que no regime de pequenas acelerações $\left(a<a_{0}\right), a=\sqrt{a_{N} a_{0}}$.

A energia escura, por sua vez, pode ser descrita no âmbito de teorias de gravidade modificada, em particular, de teorias $f(R)$, conforme já comentamos no capítulo anterior. Na próxima seção discutiremos alguns aspectos relevantes desses modelos. Em particular, desejamos mostrar como podemos acomodar um ricochete primordial de forma a resolver o problema da divergência na massa do escalaron $[57,58]$.

\subsection{Teorias $f(R)$}

A classe de modelos que vamos investigar tem uma ação da seguinte forma ${ }^{1}$ :

$$
S=\frac{1}{16 \pi G} \int f(R) \sqrt{-g} d^{4} x+S_{m}
$$

onde $f(R)$ é uma função arbitrária e não-linear do escalar de Ricci $R$ e $S_{m}$ é a ação da matéria que depende tanto da métrica como dos campos de matéria.

No formalismo métrico as equações de movimento são obtidas por variação

\footnotetext{
${ }^{1}$ Uma revisão recente sobre teorias $f(R)$ pode ser encontrada em [118]
} 
da ação (6.1) em relação ao tensor métrico ${ }^{2}$. Obtemos assim $^{3}$

$$
-\frac{1}{2} g_{\mu \nu} f(R)+f^{\prime}(R) R_{\mu \nu}-\left(\nabla_{\mu} \nabla_{\nu}-g_{\mu \nu} \square\right) f^{\prime}(R)=8 \pi G T_{\mu \nu},
$$

onde a linha denota derivada em relação ao escalar de Ricci e $T_{\mu \nu}$ é o tensor de momento e energia definido como $T_{\mu \nu}=-(2 / \sqrt{-g}) \delta S_{m} / \delta g^{\mu \nu}$.

É útil escrevermos as equações de campo para o caso particular de um espaço-tempo de FRW, cuja métrica é dada pela equação (5.7), tendo como fonte de matéria um fluido hidrodinâmico com tensor de momento e energia dado pela equação (2.13). A componente 0 - 0 leva à primeira equação de Friedmann generalizada, que é:

$$
-3\left(\dot{H}+H^{2}\right) f^{\prime}+\frac{f}{2}+3 H \frac{\partial f^{\prime}}{\partial t}=8 \pi G \rho
$$

A equação para a aceleração é obtida combinando as componentes espaciais com o traço das equações (6.2). Explicitamente, subtraindo seis vezes a componente $i-j$,

$$
\left[-\frac{f}{2}+f^{\prime}\left(2 \frac{k}{a^{2}}+3 H^{2}+\dot{H}\right)-2 H \frac{\partial f^{\prime}}{\partial t}-\frac{\partial^{2} f^{\prime}}{\partial t^{2}}\right] g_{i j}=8 \pi G P g_{i j},
$$

do traço das equações (6.2),

$$
-2 f+6 f^{\prime}\left(\dot{H}+2 H^{2}+\frac{k}{a^{2}}\right)-9 H \frac{\partial f^{\prime}}{\partial t}-3 \frac{\partial^{2} f^{\prime}}{\partial t^{2}}=-8 \pi G(\rho-3 P)
$$

obtemos a segunda equação de Friedmann generalizada, que é:

$$
-H^{2}-\frac{k}{a^{2}} f^{\prime}+\frac{f}{6}+\frac{H}{2} \frac{\partial f^{\prime}}{\partial t}+\frac{1}{2} \frac{\partial^{2} f^{\prime}}{\partial t^{2}}=-\frac{4 \pi G}{3}(\rho+3 P) .
$$

Além das equações (6.3) e (6.4), do fato da matéria aparecer minimamente acoplada com o setor gravitacional ${ }^{4}$, a conhecida equação de conservação

\footnotetext{
${ }^{2}$ Uma outra possibilidade é calcular a variação tratando tanto o tensor métrico como a conexão como variáveis independentes. Esse método é conhecido como formalismo de Palatini. Se $f(R)=R-2 \Lambda$, então a ação (6.1) se reduz à ação de Einstein-Hilbert mais um termo de constante cosmológica, caso no qual os dois formalismos coincidem. Isto se deve ao fato de que, devido às equações de movimento, a conexão se reduz à conexão de Levi-Civitta.

${ }^{3} \mathrm{~A}$ derivação detalhada encontra-se no Apêndice I.

${ }^{4} \mathrm{O}$ acoplamento mínimo corresponde, justamente, ao termo $f(R) \sqrt{-g}$ na ação (6.1).
} 
permanece válida:

$$
\dot{\rho}=-3 H(1+w) \rho .
$$

Em relatividade geral, quando o universo é preenchido por um fluido hidrodinâmico ordinário (ou seja, que obedece às condições de energia usuais, discutidas no capítulo anterior), o ricochete é possível se e somente se $k=+1$. Isto decorre imediatamente das equações (6.3) e (6.6). De fato, no ricochete, temos necessariamente $H_{b}=0$ (a partir de agora, o rótulo $b$ denota qualquer quantidade calculada no ricochete). Segue então da equação (6.3) que $f_{b}=2\left(8 \pi G \rho_{b}+3 \dot{H}_{b}\right)>0$. Portanto, da equação (6.6), temos, necessariamente, $k>0$. Mas no caso geral o último termo da equação (6.6) é diferente de zero, permitindo, desse modo, que o universo possa se contrair e se expandir na ausência de curvatura espacial e tendo como fonte de matéria um fluido perfeito que obedece às condições de energia.

Modelos $f(R)$ são, em geral, a derivadas mais altas, mas podem ser estáveis, conforme veremos. A instabilidade de teorias cujas Lagrangeanas contêm termos de derivadas de ordem superior a um está relacionada com o fato da Hamiltoniana destes sistemas ser ilimitada inferiormente [31]. Para contornar este problema, é necessário que a Lagrangeana seja degenerada, como é o caso, por exemplo, de lagrangenas da forma $\mathcal{L}=R+f(\mathrm{~GB})[119,120,121]$, onde GB se refere ao invariante topológico de Gauss-Bonnet. Antes de comentarmos com mais detalhes sobre a estabilidade de teorias $f(R)$, é útil lembrarmos o caso geral sob o qual se manifesta a instabilidade em teorias a derivadas mais altas.

\subsubsection{Instabilidade de Ostrogradski}

Vamos considerar o caso mais simples de uma Lagrangeana $\mathcal{L}$ que depende de $q$ e de suas primeira e segunda derivadas, ou seja, $\mathcal{L}=\mathcal{L}(q, \dot{q}, \ddot{q})^{5}$. Vamos também assumir que a Lagrangeana é não-degenerada, no sentido de que a definição de momento canônico $p_{2} \equiv \partial \mathcal{L} / \partial \ddot{q}$ pode ser invertida, de tal modo

\footnotetext{
${ }^{5}$ É importante notar que, se o termo de derivada segunda aparecer linearmente, podemos eliminá-lo da Lagrangeana por uma integração por partes, de tal modo que as equações de movimento se reduzem ao caso conhecido de equações de primeira ordem. De fato, temos, em geral,

$$
\int q^{n} \dot{q}^{m} \ddot{q} d t=-\frac{n}{(m+1)} \int q^{n-1} \dot{q}^{m+2} d t+\text { termos de superfície . }
$$

Nesses casos particulares, sabe-se que alguns modelos podem de fato ser estáveis [32].
} 
que $\ddot{q}$ possa ser escrito como função de $q, \dot{q}$ e $p_{2}$, ou seja,

$$
\ddot{q}=f\left(q, \dot{q}, p_{2}\right)
$$

Em 1856 [122], Ostrogradski mostrou que as quatro condições iniciais (as equações de movimento são de quarta ordem) requerem dois pares de coordenadas canônicas, por ele definidos como

$$
\begin{array}{ll}
q_{1} \equiv q & , \quad p_{1} \equiv \frac{\partial \mathcal{L}}{\partial \dot{q}}-\frac{d}{d t}\left(\frac{\partial \mathcal{L}}{\partial \ddot{q}}\right) \\
q_{2} \equiv \dot{q} & , \quad p_{2} \equiv \frac{\partial \mathcal{L}}{\partial \ddot{q}}
\end{array}
$$

A Hamiltoniana é então obtida por uma transformação de Legendre da Lagrangeana nas variáveis $\dot{q}=q^{(1)}$ e $\ddot{q}=q^{(2)}$. Ou seja,

$$
\mathcal{H}\left(p_{1}, q_{1}, p_{2}, q_{2}\right)=\sum_{i=1}^{2} p_{i} q^{(i)}-\mathcal{L}
$$

Pode-se checar explicitamente que a Hamiltoniana acima definida gera translações temporais, no sentido de que as equações canônicas

$$
\begin{aligned}
\dot{q}_{i} & =\frac{\partial \mathcal{H}}{\partial p_{i}} \\
\dot{p}_{i} & =-\frac{\partial \mathcal{H}}{\partial q_{i}}
\end{aligned}
$$

reproduzem a equação (6.8) assim como as equações de Euler-Lagrange.

O fato da Lagrangeana ser não-degenerada implica escrever $\ddot{q}$ como função de $q_{1}, q_{2}$ e $p_{2}$. Assim, podemos reescrever a Hamiltoniana (6.10) como

$$
\mathcal{H}=p_{1} q_{2}+p_{2} f\left(q_{1}, q_{2}, p_{2}\right)-\mathcal{L}(q, \dot{q}, \ddot{q})
$$

Uma característica de extrema importância da Hamiltoniana (6.12) é que ela é linear em $p_{1}$ e, portanto, ilimitada inferiormente. Em outras palavras, o sistema é necessariamente instável. É claro que sempre é possível encontrar soluções bem comportadas desse sistema, uma vez que para uma dada solução a energia é conservada. No entanto, no caso de um sistema interagente, a excitação de graus de liberdade com energia positiva será acompanhada pela excitação de graus de liberdade correspondentes com energia negativa. Este fato torna-se especialmente problemático em um sistema quântico, no qual 
o vácuo é exponencialmente instável por produção simultânea de modos de energia positiva e negativa [123].

\subsubsection{Ausência de fantasmas}

O fato de teorias $f(R)$ serem a derivadas mais altas não quer dizer, necessariamente, que a teoria seja instável. Essencialmente, isso se deve ao fato de que o novo grau de liberdade associado com a derivada de ordem mais alta se propaga com energia cinética positiva. Para compreender isso é útil reescrevermos a ação (6.1) como uma teoria escalar-tensorial ${ }^{6}$ [124]. Introduzindo o parâmetro de Lagrange $\lambda$, reescrevemos a parte gravitacional de (6.1) como

$$
\tilde{S}=\frac{1}{16 \pi G} \int\left[f(\lambda)+(R-\lambda) f^{\prime}(\lambda)\right] \sqrt{-g} d^{4} x,
$$

onde, agora, a linha denota derivada em relação a $\lambda$. Da equação de movimento para $\lambda$, que é $(R-\lambda) f^{\prime \prime}(\lambda)=0$, decorre $R=\lambda$ sempre que $f^{\prime \prime}(\lambda) \neq 0$. O próximo passo é substituir esse resultado nas equações de movimento para a métrica que seguem de (6.13). O que obtemos são precisamente as equações de movimento derivadas da ação original, equação (6.1). Em outras palavras, a ação (6.13) é equivalente a ação escalar-tensorial dada por

$$
\tilde{S}=\frac{1}{16 \pi G} \int d^{4} x \sqrt{-g}\left\{f^{\prime}(\lambda) R-0\left(\partial_{\mu} \phi\right)^{2}-\left[\lambda f^{\prime}(\lambda)-f(\lambda)\right]\right\} .
$$

Pela redefinição $f^{\prime}(\lambda) \equiv \phi$, a ação (6.14) se reduz à teoria de (Fierz-Jordan)Brans-Dicke $[125,126,127]$ com parâmetro de Brans-Dicke $\omega_{B D}=0$, e com um potencial definido pelo termo entre colchetes.

Contrariamente ao que poderia indicar a ausência de um termo cinético explícito para $\phi$ na Lagrangeana, este campo constitui de fato um grau de liberdade dinâmico. Podemos antecipar isso por inspeção da parte não-minimamente acoplada da ação (6.14), que depende de termos da forma $\phi \partial^{2} g$, que vão gerar, por uma integração por partes, termos como $-\partial \phi \partial g$. A equação que governa a evolução de $\phi$ é, precisamente, o traço das equações de movimento, equações

\footnotetext{
${ }^{6}$ Isto é sempre possível desde que $f^{\prime \prime}(R) \neq 0$. Se $f^{\prime \prime}(R)=0$, então há duas possibilidades, quais sejam, ou $f(R)$ é constante e, nesse caso, não há grau de liberdade gravitacional, ou $f(R)=R-2 \Lambda$, ou seja, a teoria é a relatividade geral com uma possível constante cosmológica.
} 
(6.5), que também pode ser escrita da seguinte forma elucidativa:

$$
\square \phi-W_{, \phi}=\frac{8 \pi G}{3} T
$$

onde $T=g^{\mu \nu} T_{\mu \nu} \mathrm{e}$

$$
W_{, \phi} \equiv \frac{2 f-f^{\prime} R}{3} .
$$

A equação (6.15) nos diz que $\phi$ se propaga através de um potencial efetivo $W$ e cuja fonte é dada pelo traço do tensor de momento e energia.

Com o intuito de diagonalizarmos os termos cinéticos da Lagrangeana, é útil escrevermos a ação (6.14) em uma forma conveniente pela seguinte transformação de variáveis [32]:

$$
\begin{aligned}
g_{\mu \nu}^{*} & =f^{\prime}(\lambda) g_{\mu \nu} \\
\varphi & =\frac{\sqrt{3}}{2} \ln f^{\prime}(\lambda) \\
V(\varphi) & =\frac{\lambda f^{\prime}(\lambda)-f(\lambda)}{4 f^{\prime 2}(\lambda)}
\end{aligned}
$$

Portanto, a ação (6.14) mais a parte da matéria fica

$$
S=\frac{1}{16 \pi G} \int d^{4} x \sqrt{-g^{*}}\left\{\frac{R^{*}}{4}-\frac{1}{2} g_{*}^{\mu \nu} \partial_{\mu} \varphi \partial_{\nu} \varphi-V(\varphi)\right\}+S_{\mathrm{m}}\left[\psi, g_{\mu \nu}=A^{2}(\varphi) g_{\mu \nu}^{*}\right]
$$

onde $\psi$ denota coletivamente os campos de matéria e $A(\varphi)=e^{\varphi / 3}$. Com a ação escrita na forma acima, fica portanto evidente o fato de que o campo $\varphi$ se propaga com uma energia cinética positiva ${ }^{7}$. A estabilidade, no entanto, não fica garantida a menos que o potencial $V(\varphi)$ também seja limitado inferiormente, condição esta que se traduz em vínculos sobre a forma original da função $f(R)$.

Na próxima subseção discutiremos os vínculos de estabilidade, assim como os vínculos cosmológicos e de testes locais de gravidade, que modelos viáveis de gravidade $f(R)$ devem satisfazer.

\footnotetext{
${ }^{7}$ Aqui é útil lembrarmos a assinatura da métrica $g_{\mu \nu}^{*}$ : assinatura $g_{\mu \nu}^{*}=(-,+,+,+)$. É importante notar que assinatura $g_{\mu \nu}=(-,+,+,+)$, o que implica $f^{\prime}(\lambda)>0$ para que não haja "ghosts".
} 


\subsubsection{Vínculos importantes}

Um modo conveniente de se comparar a relatividade geral com os experimentos de sistema solar é em termos dos chamados parâmetros de Eddington [33] $\gamma$ e $\beta$, definidos como sendo os coeficientes da expansão pós-newtoniana do espaçotempo de Schwarzschild:

$$
\begin{aligned}
-g_{00} & \approx 1-\frac{r_{s}}{r}+\frac{\beta-\gamma}{2} \frac{r_{s}^{2}}{r^{2}} \\
g_{11} & \approx 1+\gamma \frac{r_{s}}{r}
\end{aligned}
$$

onde $r_{s}$ é o raio de Schwarzschild. A solução exata de Schwarzschild corresponde a

$$
\beta=\gamma=1
$$

A relatividade geral pode então ser testada verificando-se o quão próximos da unidade são os parâmetros de Eddington. Por medições do atraso de Shapiro [33], o "Viking Project" obteve em $1993 \gamma=1,000 \pm 0,001$. Um valor mais recente, de 2003, foi obtido por observações da sonda Cassini [128], que é:

$$
\gamma=1,000000 \pm 0,000023
$$

Podemos usar o valor acima para $\gamma$ para vincular o parâmetro $\omega_{B D}$. No caso de teorias escalar-tensoriais, $\gamma$ pode ser escrito como $[129,130]$

$$
\gamma=1-\frac{2}{3+2 \omega_{B D}}
$$

Ou seja, no limite $\omega_{B D} \rightarrow \infty$ a teoria se reduz à relatividade geral. Levando-se em conta (6.20) e (6.21), devemos ter

$$
\omega_{B D} \gtrsim 40000
$$

Como vimos na subseção precedente, teorias $f(R)$ podem ser escritas como teorias escalar-tensoriais com parâmetro de Brans-Dicke identicamente nulo mas com um potencial. Esta discordância entre $\omega_{B D}$, entretanto, não quer dizer que modelos baseados em gravidade $f(R)$ estão automaticamente descartados. Isto se deve ao fato de que as previsões da teoria de Brans-Dicke dizem respeito apenas a campos escalares não-massivos. O potencial desem- 
penha, de fato, um papel chave, pois permite que o campo escalar adquira uma massa variável (dependente da densidade de matéria local), de tal modo que, em escalas típicas do sistema solar (alta densidade local), seu efeito é imperceptível, contrariamente ao o que ocorre em escalas cosmológicas (baixa densidade local). Assim, de uma certa forma, podemos dizer que a natureza escalar-tensorial da gravidade se manifesta separadamente em escalas distintas: em pequenas escalas, ela é, essencialmente, de natureza tensorial, ao passo que, em grandes escalas, é seu caráter escalar que predomina. Esse comportamento mutante do campo entre estados massivos e pouco massivos é conhecido como mecanismo do camaleão [131, 132, 133, 134]. Levando-se em conta este mecanismo, o vínculo de testes locais de gravidade se traduz pela seguinte desigualdade em termos das derivadas da função $f(R)[56,57,135]$ :

$$
0<m \ll 1
$$

onde $m \equiv R f^{\prime \prime} / f^{\prime}$.

Além de possuir um espaço de fase com um atrator de de Sitter, modelos $f(R)$ também devem reproduzir as eras cosmológicas do modelo padrão afim de que vínculos de nucleossíntese primordial e de formação de estruturas sejam satisfeitos. Conforme mostraram Amendola, Polarski e Tsujikawa [136], isto ocorrerá contanto que

$$
m>0, \quad-1<\frac{d m}{d r}<0, \quad \text { em }(r, m) \approx(-1,0),
$$

onde $r \equiv-R f^{\prime} / f$ com $f^{\prime}$ da ordem da unidade durante a era dominada pela matéria. Além disso, para que o atrator de de Sitter seja estável, é necessário que [136]

$$
r=-2,0<m \leq 1
$$

Em teorias escalar-tensoriais, podemos definir uma "constante" gravitacional efetiva dependente do tempo $G_{e f} \equiv G / f^{\prime}(\lambda)$. Essa variação temporal, no entanto, deve ser muito pequena, como indicam as observações do "Viking Project" [137] e de pulsares binários [138] que estabelecem os seguintes limites superiores para $\dot{G}_{e f} / G_{e f}$ :

$$
\frac{\dot{G}_{e f}}{G_{e f}}=\left\{\begin{array}{cc}
(0,2 \pm 0,4) \times 10^{-11} / \text { ano } & \text { (Viking Project) } \\
(-0,06 \pm 0,2) \times 10^{-11} / \text { ano } & \text { (Pulsares binários) }
\end{array}\right.
$$




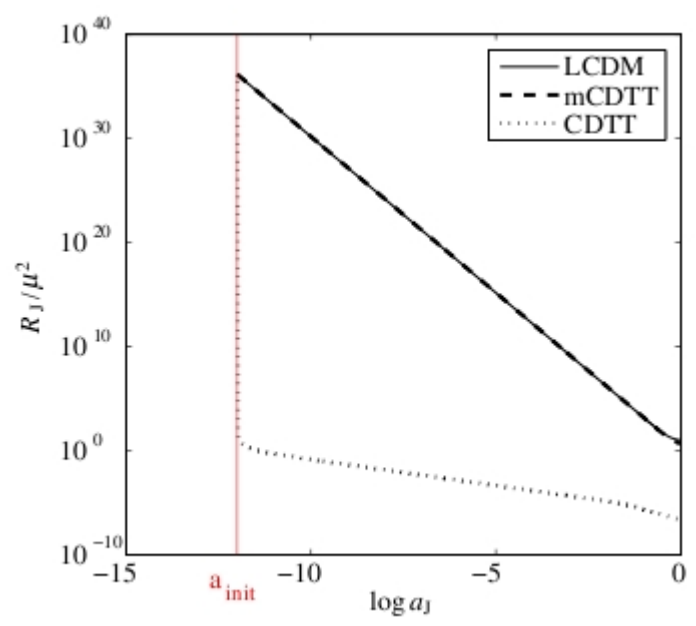

Figura 6.1: Gráfico extraído de [140]. O eixo vertical representa o escalar de Ricci $R_{J}$ no quadro de Jordan normalizado em relação a $\mu \sim H_{0}$. O eixo horizontal representa o fator de escala $a_{J}$, também no quadro de Jordan. Temos 3 classes de modelo representadas: LCDM, que corresponde ao modelo da concordância $\Lambda$ CDM; mCDTT, que corresponde ao modelo $f(R)$ (estável) introduzido por Carroll, Dovvuri, Troden e Turner (CDTT) [141] e, finalmente, modelos (instáveis) CDTT [141]. O modelo mCDTT concorda com o modelo da concordância, enquanto que no modelo CDTT a solução é instável, e o escalar de Ricci cai a um valor bem menor do que a solução correspondente da relatividade geral. A fase que se segue é dominada pelo grau de liberdade escalar e substitui, nesses modelos, a fase usual dominada pela matéria.

Também, para que o caráter atrativo da gravidade seja preservado $\left(G_{e f}>0\right)$, devemos ter ${ }^{8}$

$$
f^{\prime}>0
$$

Além de ser consistentes com testes locais de gravidade e satisfazer vínculos cosmológicos, modelos $f(R)$ também devem ser estáveis por perturbações lineares. Dolgov e Kawasaki [139] mostraram que Lagrangeanas da forma $\sim 1 / R$ , com $f^{\prime \prime}<0$, resultam em soluções de campo fraco em regiões internas a uma distribuição de matéria que divergem rapidamente. Por outro lado, em regimes de alta curvatura, conforme demonstraram Hu e Sawicki [140], a instabilidade em modelos com $f^{\prime \prime}<0$ leva o universo a evoluir por uma fase dominada pelo grau de liberdade escalar na qual a curvatura assume valores bem inferiores aos valores do modelo cosmológico padrão (ver figura (6.1)), comportamento este que é inconsistente com os vínculos cosmológicos.

A instabilidade de modelos com $f^{\prime \prime}<0$ pode ser entendida por meio da equação dinâmica para o campo escalar, equação (6.15). Da definição do po-

\footnotetext{
${ }^{8}$ Condição esta necessária para que o gráviton não seja um "ghost". Ver nota 7.
} 
tencial $W_{, \phi}$, vemos que a massa do campo pode ser escrita como

$$
M_{\phi}^{2} \equiv W_{, \phi \phi}=\frac{1}{3}\left(\frac{f^{\prime}}{f^{\prime \prime}}-R\right)
$$

Logo, se $f^{\prime}>0$ e $R>0, f^{\prime \prime}<0$ implica $M_{\phi}^{2}<0$, refletindo o caráter taquiônico e de rápido rolamento ${ }^{9}$ do campo, além de implicar violação de testes locais de gravidade, como podemos verificar da condição $0<m=R f^{\prime \prime} / f^{\prime}<<1$.

As considerações acima implicam, portanto, o seguinte vínculo de estabilidade:

$$
f^{\prime \prime}>0
$$

Modelos que satisfazem aos vínculos acima têm sido propostos, notavelmente em [57] e [56]. Em um regime de alta curvatura, eles podem ser escritos genericamente como [58]

$$
f(R) \simeq R-\lambda R_{c}\left[1-\left(\frac{R_{c}}{R}\right)^{2 n}\right],
$$

onde $n, \lambda$ e $R_{c}$ são constantes positivas e $R_{c}$ é da ordem de $R_{0}$ (a curvatura escalar hoje) para $\{n, \lambda\} \sim \mathcal{O}(1)$, caso para o qual temos ainda

$$
m(r)=C(-r-1)^{2 n+1}
$$

onde $C$ é uma constante positiva ${ }^{10}$. Durante as eras da radiação e matéria, como podemos ver das desigualdades (6.24), $m \rightarrow 0$, o que quer dizer que esses modelos são muito parecidos com o modelo cosmológico padrão com constante cosmológica e matéria escura fria ${ }^{11}$ [140].

A hipótese de que, devido à natureza escalar-tensorial da gravidade, o universo presente esteja passando por uma fase de expansão acelerada nos remete naturalmente à questão de como o campo escalar deve ter se comportado em épocas primordiais. Conforme mostrou Starobinsky [57], o fato da amplitude de modos oscilatórios do escalar de Ricci crescerem para o passado (e divergi-

\footnotetext{
${ }^{9}$ Runaway, em inglês.

${ }^{10}$ Note que as condições de estabilidade $f^{\prime}(R)>0$ e $f^{\prime \prime}(R)>0, R \geq R_{c}>0$, não são impostas sobre todo o espaço de soluções onde $R$ pode assumir qualquer valor, inclusive valores negativos, mas aplicam-se apenas sobre uma trajetória da história cósmica que se inicia em um regime de alta curvatura, $\operatorname{com} R \gg R_{c}$, e evolui para um estado de baixa curvatura, para o qual $R \sim R_{c}$.

${ }^{11}$ Também conhecido como modelo da concordância, ou $\Lambda$ CDM ( $\Lambda$ "Cold Dark Matter").
} 
rem em $t=0$ ) implica violação da condição (6.28). Mas isto significa que, se $f^{\prime \prime}<0$ em algum momento, então a função $f$ evoluiu, necessariamente, através do ponto $f^{\prime \prime}=0$ onde $M_{\phi}^{2} \rightarrow \infty$. Surge assim uma importante questão, a saber, como evitar que a produção de partículas escalares implique violação da desigualdade $f^{\prime \prime}>0$. Antes de estudarmos esse problema no âmbito de ricochetes cosmológicos, devemos, antes de mais nada, verificar se as soluções de ricochete são elas mesmas consistentes com a condição (6.28).

\subsubsection{Solução de ricochete}

Vamos considerar a situação mais simples de um universo homogêneo e isotrópico, espacialmente plano, e preenchido por um fluido hidrodinâmico ordinário. Nesse caso, com a substituição $f^{\prime} \equiv \phi$ e $k=0$, as equações (6.3) e (6.5) se escrevem como

$$
\begin{aligned}
-3\left(\dot{H}+H^{2}\right)+ & \frac{1}{\phi}\left(3 H \dot{\phi}+\frac{f}{2}\right)=\frac{8 \pi G \rho}{\phi} \\
\ddot{\phi}+3 H \dot{\phi}+W_{, \phi} & =-\frac{8 \pi G}{3} T
\end{aligned}
$$

O traço do tensor de momento e energia é $T=\rho(3 w-1)$. Além das equações (6.31) e (6.32), há a seguinte equação da continuidade:

$$
\dot{\rho}=-3 H(1+w) \rho
$$

Vamos também assumir que a função $f(R)$ é analítica em $R_{b}=6 \dot{H}_{b}=$ $6 \ddot{a}_{b} / a_{b}>0$, o escalar de Ricci no ricochete (o rótulo $b$, daqui em diante, denota quantidades calculadas no ricochete), de tal modo que vale a seguinte expansão para $f(R)$ :

$$
f(R)=f_{b}+f_{1}\left(R-R_{b}\right)+f_{2}\left(R-R_{b}\right)^{2}+f_{3}\left(R-R_{b}\right)^{3}+\mathcal{O}\left[\left(R-R_{b}\right)^{4}\right]
$$

De modo análogo, escrevemos o parâmetro de Hubble, o campo escalar e a densidade de energia como expansões em torno de $t_{b}$, que escolhemos como sendo 0. O que obtemos então das equações de movimento (6.31), (6.32) e (6.33) são relações entre os coeficientes das expansões $\left(f_{1}, f_{2}\right.$ e $f_{3}$, no caso da função $f(R)$ ) e as condições iniciais fixadas em $t=0^{12}$.

\footnotetext{
${ }^{12} \mathrm{O}$ cálculo detalhado está no Apêndice II.
} 
Para o parâmetro de Hubble obtemos

$$
H(t)=\frac{f_{b}-16 \mathcal{P}_{b}}{6 \phi_{b}} t\left[1+\varepsilon(1+w) \sqrt{\frac{\mathcal{P}_{b}}{\phi_{b} \gamma_{b}(w)}} t\right]+\mathcal{O}\left(t^{3}\right),
$$

onde definimos $\varepsilon= \pm 1, \mathcal{P}_{b} \equiv \pi G \rho_{b}$ e o seguinte número $\gamma_{b}(w)$ :

$$
\gamma_{b}(w) \equiv \frac{2\left[f_{b}+4 \mathcal{P}_{b}(3 w+7)(3 w+2)\right]}{f_{b}-16 \mathcal{P}_{b}}
$$

O termo de ordem zero da solução (6.35) é, por hipótese, igual a zero, pois em $t=0$ temos, obviamente, $\dot{a}_{b}=0$ e $\ddot{a}_{b}=a_{b} \dot{H}_{b}>0$. Além disso, se a desigualdade $f^{\prime}>0$ for válida, então $\phi>0$. Destas considerações e da equação (6.35) decorre a seguinte condição de ricochete:

$$
f_{b}>16 \mathcal{P}_{b}
$$

Da desigualdade (6.37), segue também que $\gamma_{b}(w)>0$.

A solução para $\phi$ é:

$$
\begin{array}{r}
\phi(t)=\phi_{b}-4 \varepsilon(1+w) \sqrt{\mathcal{P}_{b} \phi_{b} \gamma_{b}(w)} t-\frac{1}{6}\left[f_{b}+24 \mathcal{P}_{b}\left(w+\frac{1}{3}\right)\right] t^{2}+ \\
+\varepsilon \frac{(1+w)}{9 \gamma_{b}(w)} \sqrt{\frac{\mathcal{P}_{b}}{\phi_{b}}}\left(f_{b}-16 \mathcal{P}_{b}\right) t^{3}+\mathcal{O}\left(t^{4}\right) .
\end{array}
$$

Como $f^{\prime}>0$, é claro que $\phi_{b}>0$.

Para a densidade de energia obtemos

$$
\rho(t)=\rho_{b}\left[1-\frac{f_{b}-16 \mathcal{P}_{b}}{\phi_{b}}\left(\frac{(1+w)}{4} t^{2}+\frac{\varepsilon(1+w)^{2}}{6 \gamma_{b}^{1 / 2}(w)} \sqrt{\frac{\mathcal{P}_{b}}{\phi_{b}}} t^{3}\right)+\mathcal{O}\left(t^{4}\right)\right]
$$

Em uma fase de contração, é de se esperar que a densidade de energia cresça gradualmente até atingir um valor máximo em $t=0$, instante a partir do qual ela passa a diminuir. Tal comportamento se reflete na solução (6.39), com o termo de primeira ordem, $\rho_{1}$, sendo igual a zero e o termo de segunda ordem, $\rho_{2}$, negativo. $\mathrm{O}$ termo de terceira ordem $\rho_{3} \neq 0$ exprime o fato de que o ricochete é não simétrico. 

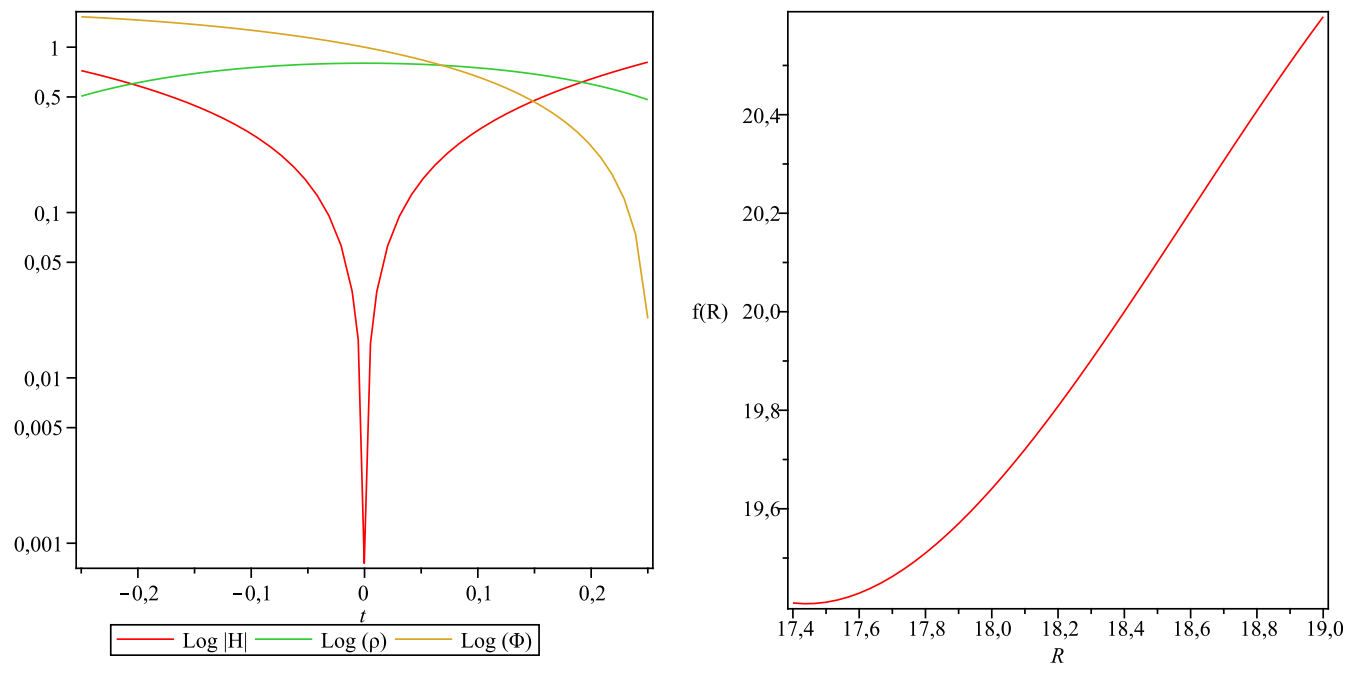

Figura 6.2: Soluções para $H, \phi, \rho$ e $f(R)$ nas vizinhanças de $t=0, \operatorname{com} 8 \pi G \equiv 1$ e: $w=1 / 3, f_{b}=20$, $\mathcal{P}_{b}=0,1, \rho_{b}=8 \mathcal{P}_{b}=0,8, \phi_{b}=1$ e $\varepsilon=+1$. Em $t=0, R_{b}=18,4$.

Finalmente, para a função $f(R)$, obtemos

$$
\begin{aligned}
f(R)=f_{b} & +\phi_{b}\left(R-R_{b}\right)+\frac{\phi_{b}^{2} \gamma_{b}(w)}{f_{b}-16 \mathcal{P}_{b}}\left(R-R_{b}\right)^{2}- \\
& -\frac{\phi_{b}^{3} \gamma_{b}(w) \delta_{b}(w)}{24 \mathcal{P}_{b}(1+w)^{2}\left(f_{b}-16 \mathcal{P}_{b}\right)}\left(R-R_{b}\right)^{3}+\mathcal{O}\left[\left(R-R_{b}\right)^{4}\right],
\end{aligned}
$$

onde definimos

$$
\delta_{b}(w) \equiv \frac{3 f_{b}+8 \mathcal{P}_{b}\left(12 w^{2}+37 w+19\right)}{f_{b}-16 \mathcal{P}_{b}}>0 .
$$

Portanto,

$$
f^{\prime \prime}=\frac{2 \phi_{b}^{2} \gamma_{b}(w)}{f_{b}-16 \mathcal{P}_{b}}+\mathcal{O}\left[\left(R-R_{b}\right)\right]>0
$$

A figura (6.2) contem as soluções para $H, \phi, \rho$ e $f(R)$. Está claro que as desigualdades $f^{\prime}>0$ e $f^{\prime \prime}>0$ são ambas satisfeitas nas vizinhanças de $t=0$.

Tendo verificado que o vínculo de estabilidade é satisfeito perto de $t=0$, passemos à parte seguinte, onde estudaremos a evolução da perturbação da curvatura escalar durante essa fase. 


\subsubsection{Perturbações de densidade}

Vamos iniciar esta seção introduzindo as equações para as perturbações de densidade em teorias $f(R)$. Um estudo sobre este assunto pode ser encontrado em [142].

Em um espaço-tempo de FRW com curvatura espacial nula, as perturbações escalares da métrica $\alpha, b, \varphi$ e $E$ são definidas como [143, 65]

$$
d s^{2}=-(1+2 \alpha) d t^{2}-2 a b_{, j} d t d x^{j}+a^{2}(t)\left[(1+2 \varphi) \delta_{i j}+2 E_{; i j}\right] d x^{i} d x^{j} .
$$

Vamos nos restringir ao caso particular em que $P$, a pressão do fluido, é nula. Nesse caso, o tensor de momento e energia pode ser decomposto como ${ }^{13}$

$$
\begin{aligned}
T_{0}^{0} & =-\left(\rho_{m}+\delta \rho_{m}\right) \\
T_{i}^{0} & =-\rho_{m} v_{m, i} \\
T_{j}^{i} & =0
\end{aligned}
$$

onde $v_{m, i}$ é uma definição covariante da variável de velocidade [144].

No espaço de Fourier, para um número de onda comóvel $k$, as perturbações da matéria satisfazem as seguintes equações [142]:

$$
\begin{aligned}
\delta \dot{\rho}_{m}+3 H \delta \rho_{m} & =\rho_{m}\left(\kappa-3 H \alpha-\frac{k^{2}}{a} v_{m}\right) \\
\dot{v}_{m}+H v_{m} & =\frac{\alpha}{a}
\end{aligned}
$$

onde $\kappa \equiv 3(H \alpha-\dot{\varphi})+\frac{k}{a^{2}} \chi$ e $\chi \equiv a(b+a \dot{E})$. Com as definições do potencial de velocidade $v \equiv a v_{m}$ e do contraste de densidade $\delta \equiv \frac{\delta \rho_{m}}{\rho_{m}}$, as equações (6.45) são escritas como

$$
\begin{aligned}
\dot{\alpha} & =\dot{v} \\
\dot{\delta} & =\kappa-3 H \alpha-\frac{k}{a^{2}} v
\end{aligned}
$$

Usando o contraste de densidade e o potencial de velocidade podemos construir a quantidade invariante de calibre $\delta_{m}=\frac{\delta \rho_{m}}{\rho_{m}}+3 H v$, cuja equação de movimento

\footnotetext{
${ }^{13}$ Nesta seção, $8 \pi G \equiv 1$.
} 
segue imediatamente de (6.46) e é dada por

$$
\ddot{\delta}_{m}+2 H \dot{\delta}_{m}+\frac{k}{a^{2}}(\alpha-\dot{\chi})=3(H v-\varphi)^{*}+6 H(H v-\varphi)^{.}
$$

Agora, vamos reproduzir as equações para as perturbações escalares. Em uma forma geral, isto é, sem eleger um calibre em particular, são elas [142, 135]:

- Equação $G_{0}^{0}$ :

$$
-\frac{k^{2}}{a^{2}} \varphi+\left(H+\frac{\dot{F}}{2 F}\right) \kappa+\frac{3 H \dot{F}}{2 F} \alpha=-\frac{1}{2 F}\left[\delta \rho_{m}-3 H \delta \dot{F}+\left(3 \dot{H}+3 H^{2}-\frac{k^{2}}{a^{2}}\right) \delta F\right]
$$

- Equação $G_{i}^{0}$ :

$$
H \alpha-\dot{\varphi}=\frac{1}{2 F}\left[\delta \dot{F}-H \delta F-\dot{F} \alpha+\rho_{m} v\right]
$$

- Equação $G_{j}^{i}-\frac{1}{3} \delta_{j}^{i} G_{k}^{k}$ :

$$
\dot{\chi}+\left(H+\frac{\dot{F}}{F}\right) \chi-\alpha-\varphi=\frac{\delta F}{F}
$$

- Equação $G_{k}^{k}-G_{0}^{0}$

$$
\begin{array}{r}
\dot{\kappa}+2 H \kappa+\left(3 \dot{H}-\frac{k^{2}}{a^{2}}\right) \alpha=\frac{1}{2 F}\left[\left(-6 H^{2}+\frac{k^{2}}{a^{2}}\right) \delta F+3 H \delta \dot{F}+\right. \\
\left.+3 \delta \ddot{F}-\dot{F} \kappa-3(2 \ddot{F}+H \dot{F}) \alpha-3 \dot{F} \dot{\alpha}+\delta \rho_{m}\right]
\end{array}
$$

- Equação $G_{\lambda}^{\lambda}$

$$
\delta \ddot{F}+3 H \delta \dot{F}+\left(\frac{k^{2}}{a^{2}}-\frac{R}{3}\right) \delta F=\frac{1}{3} \delta \rho_{m}+\dot{F}(\kappa+\dot{\alpha})+(2 \ddot{F}+3 H \dot{F}) \alpha-\frac{1}{3} F \delta R
$$

Nas equações acima, $F \equiv \partial f / \partial R$.

É conveniente definirmos um calibre com o intuito de simplificarmos as equações (6.47) - (6.52). Exemplos de calibres conhecidos são: calibre síncrono $(\alpha=0)$, comóvel $(v=0)$ e longitudinal $(\chi=0)$. No calibre comóvel, como 
$v=0$, então $\alpha=0$ e $\dot{\delta}_{m}=\kappa$, conforme segue das equações (6.46) e da definição de $\delta_{m}$. As equações (6.51) e (6.52) ficam então:

$$
\begin{gathered}
\ddot{\delta}_{m}+\left(2 H+\frac{\dot{F}}{2 F}\right) \dot{\delta}_{m}=\frac{1}{2 F}\left[\left(-6 H^{2}+\frac{k^{2}}{a^{2}}\right) \delta F+3 H \delta \dot{F}+3 \delta \ddot{F}+\delta \rho_{m}\right] \\
\delta \ddot{F}+3 H \delta \dot{F}+\left(\frac{k^{2}}{a^{2}}+\frac{f^{\prime}}{3 f^{\prime \prime}}-4 H^{2}-2 \dot{H}\right) \delta F=\frac{1}{3} \delta \rho_{m}+\dot{F} \dot{\delta}_{m}
\end{gathered}
$$

Na equação (6.56) usamos o fato de que $\delta R=\delta F / f^{\prime \prime}$. Também, os três últimos termos do lado esquerdo da equação (6.56) constituem nada mais do que a massa de $\phi$ definida em (6.27), que pode ser aproximada para

$$
M_{\phi}^{2}=\frac{f^{\prime}}{3 f^{\prime \prime}}-4 H^{2}-2 \dot{H} \approx \frac{f^{\prime}}{3 f^{\prime \prime}}
$$

por consistência com testes locais de gravidade (ver equação (6.23)). Além disso, como $m \rightarrow 0$, então segue dos vínculos cosmológicos que $|\dot{F} / H F| \ll$ 1. Levando-se em conta estas aproximações, podemos reescrever as equações (6.53) e (6.56) como

$$
\begin{aligned}
& \ddot{\delta}_{m}+2 H \dot{\delta}_{m}=\frac{1}{2 F}\left[\left(-6 H^{2}+\frac{k^{2}}{a^{2}}\right) \delta F+3 H \delta \dot{F}+3 \delta \ddot{F}+\delta \rho_{m}\right] \\
& \delta \ddot{F}+3 H \delta \dot{F}+\left(\frac{k^{2}}{a^{2}}+M_{\phi}^{2}\right) \delta F=\frac{1}{3} \delta \rho_{m}
\end{aligned}
$$

Os termos remanescentes na equação (6.56) também indicam que estamos no regime de pequenos comprimentos de onda, ou seja, em um regime tal que $k^{2} / a^{2} \gg\left\{H^{2}, \dot{H}\right\}$.

A solução da equação (6.56) pode ser escrita como a soma de dois modos [145]: o primeiro deles, não-oscilatório, é devido à presença da perturbação da matéria, e o segundo modo, oscilatório, corresponde à solução da equação homogênea $\left(\delta \rho_{m}=0\right)$. A solução do primeiro modo é obtida desprezando-se tanto o termo de fricção como o termo responsável pelas oscilações de $\delta F$. Ou seja,

$$
\delta R \simeq \frac{\delta \rho_{m}}{F(1+3 \xi)}
$$

onde

$$
\xi \equiv \frac{k^{2}}{a^{2}} \frac{f^{\prime \prime}}{f^{\prime}}=\frac{k^{2}}{a^{2} R} m
$$


O modo oscilatório, por sua vez, satisfaz a seguinte equação:

$$
\delta \ddot{F}+3 H \delta \dot{F}+\left(\frac{k^{2}}{a^{2}}+M_{\phi}^{2}\right) \delta F \simeq 0 .
$$

Em regimes de pequenos comprimentos de onda nos quais $\left\{k^{2} / a^{2}, M_{\phi}^{2}\right\} \gg H^{2}$ podemos reescrever a equação acima em uma forma mais elucidativa como

$$
\left(a^{3 / 2} \delta F\right)^{*}+\omega^{2}\left(a^{3 / 2} \delta F\right) \simeq 0
$$

onde $\omega \equiv \sqrt{k^{2} / a^{2}+M_{\phi}^{2}}$. Vamos supor que a seguinte desigualdade é verdadeira: $|\dot{\omega} / \omega| \ll 1$. Então a equação (6.59) admite a seguinte solução WKB:

$$
\delta F \simeq \frac{c a^{-3 / 2}}{\sqrt{2 \omega}} \cos \left(\int \omega d t\right)
$$

onde $c$ é uma constante arbitrária. Chegamos assim à seguinte solução para $\delta R$ :

$$
\delta R \simeq \frac{\delta \rho_{m}}{f^{\prime}(1+3 \xi)}+\frac{c a^{-3 / 2}}{f^{\prime \prime} \sqrt{2 \omega}} \cos \left(\int \omega d t\right)
$$

Vamos agora investigar o comportamento da amplitude do termo oscilatório em (6.60). Em modelos $f(R)$ viáveis, durante a era dominada pela matéria o fator de escala cresce como $t^{2 / 3}$, enquanto que o escalar de Ricci de fundo, $\tilde{R}$, decresce como $t^{-2}$. Mas, da equação (6.29), temos $f^{\prime \prime} \propto \tilde{R}^{-2(n+1)}$, de tal modo que $M_{\phi}^{2} \simeq 1 / f^{\prime \prime} \simeq t^{-4(n+1)}$. Por outro lado, está claro que, à medida que retrocedemos no tempo, o termo de massa progressivamente domina sobre o termo $k^{2} / a^{2}$, porque $k^{2} / a^{2} \propto t^{-4 / 3}$ e $M_{\phi}^{2} \propto t^{-4(n+1)}$ com $n \sim \mathcal{O}(1)$ (ver equação (6.29)). Coletando os resultados acima, concluímos que

$$
\left|\delta R_{o s c}\right| \propto c t^{-(3 n+2)}
$$

ou seja, em um tempo suficientemente distante para o passado, a amplitude do modo oscilatório $\delta R_{\text {osc }}$ domina sobre o escalar de Ricci de fundo $\tilde{R}$, violando assim a hipótese segundo a qual $\left|\delta R_{o s c}\right| \ll \tilde{R}$. Mais ainda, devido ao crescimento da amplitude de oscilação, o escalar de Ricci $R=\tilde{R}+\delta R_{\text {ind }}+\delta R_{\text {osc }}$, onde $\delta R_{\text {ind }}$ é dado pelo primeiro termo da solução (6.60), pode se tornar menor que $R_{c}$ (ver equação (6.29)), ou até mesmo negativo, durante uma oscilação, 
violando dessa forma a condição de estabilidade $f^{\prime \prime}>0{ }^{14}$. Do ponto de vista cosmológico, isto significa que o modelo não possui uma era da matéria (e tampouco uma era da radiação) suficientemente longa, uma vez que a solução atinge o ponto singular $f^{\prime \prime}=0$ em um tempo passado finito [62 ${ }^{15}$.

Uma maneira de se "resolver" este problema é impondo que $c$ seja tão pequeno quanto for necessário para que a condição $\left|\delta R_{o s c}\right|<\tilde{R}$ seja satisfeita desde o início da era da radiação. No entanto, este ajuste fino implicaria ao mesmo tempo a eliminação quase completa do grau de liberdade escalar.

Vemos então que algum processo deve ocorrer no universo jovem para que a produção de escalarons por oscilações do escalar de Ricci não implique violações da desigualdade $f^{\prime \prime}>0$. Vamos supor que esse processo é descrito por um ricochete do fator de escala. Já sabemos da seção precedente que, desde que a Lagrangeana seja analítica em $t=0$, a condição $f^{\prime \prime}>0$ é satisfeita em torno desse instante. Temos então:

$$
\begin{aligned}
a(t) & =a_{b}\left(1+\frac{f_{b}-16 \mathcal{P}_{b}}{12 \phi_{b}} t^{2}+\mathcal{O}\left(t^{3}\right)\right) \\
\left|\delta R_{o s c}\right| & =\sqrt{\frac{f_{b}-16 \mathcal{P}_{b}}{4 a_{b}^{3} \phi_{b}^{2} \gamma_{b}(w)}}\left(1-\frac{f_{b}-16 \mathcal{P}_{b}}{8 \phi_{b}} t^{2}+\mathcal{O}\left(t^{3}\right)\right) \\
\tilde{R}(t) & =\frac{f_{b}-16 \mathcal{P}_{b}}{a_{b} \phi_{b}}\left(1+\frac{f_{b}-16 \mathcal{P}_{b}}{12 a_{b} \phi_{b}} t^{2}+\mathcal{O}\left(t^{3}\right)\right) .
\end{aligned}
$$

Como podemos ver pela figura (6.3) representando as soluções (6.62), (6.63) e (6.64), a condição $\left|\delta R_{o s c}\right|<\tilde{R}$ é satisfeita sempre que o valor mínimo do fator de escala for maior que um certo valor dado por:

$$
a_{b}>\left[2\left(f_{b}-16 \mathcal{P}_{b}\right) \gamma_{b}(w)\right]^{-1 / 3}
$$

conforme segue das equações (6.63) e (6.64).

Tendo estabelecido que uma fase de contração seguida de expansão oferece um mecanismo para impedir que a produção de escalarons viole a condição de estabilidade $f^{\prime \prime}>0$, resta saber como conectar a fase de ricochete com modelos

\footnotetext{
${ }^{14} \mathrm{Um}$ comportamento similar ocorre também na era dominada pela radiação [57].

${ }^{15}$ Para uma possível solução para o problema da singularidade em modelos $f(R)$ assinalado em [62], ver [59]. Este modelo, no entanto, não satisfaz testes locais de gravidade [60] nem vínculos observacionais de estruturas de grandes escalas [61]. Uma outra possibilidade [146] é adicionando um termo de correção $\beta R^{2}$ na Lagrangeana. Nesse caso, apesar do modo oscilatório do escalaron ser subdominante no passado, o termo de correção quadrático leva o escalar de curvatura a cruzar o ponto $f^{\prime \prime}=0$ no futuro.
} 


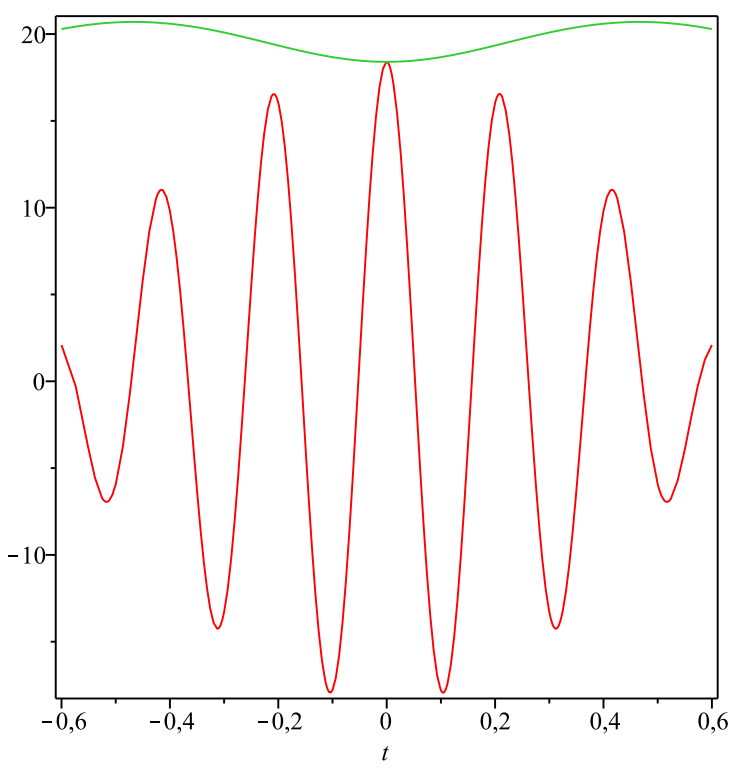

Figura 6.3: Solução para $\delta R_{o s c}$ (linha vermelha). A linha verde corresponde à solução de fundo para o escalar de Ricci. Usamos os mesmos valores da figura (6.2), que são: $w=1 / 3, f_{b}=20, \mathcal{P}_{b}=0,1$, $\rho_{b}=8 \mathcal{P}_{b}=0,8$ e $\phi_{b}=1$.

$f(R)$ viáveis, como, por exemplo, os modelos definidos pela equação (6.30). Esta importante questão permanece sob investigação.

\subsection{Outras teorias a derivadas mais altas}

Teorias $f(R)$ constituem a forma mais simples de generalização da ação de Einstein-Hilbert. Uma característica importante dessa classe de modelos é o fato de que suas soluções podem ser estáveis. Sob certas condições, também podem obedecer a testes locais de gravidade e a vínculos cosmológicos. Por outro lado, a existência de singularidades ainda é problemática, notavelmente no que se refere a divergências no escalar de curvatura em épocas primordiais. Essa questão nos leva naturalmente a pensar em teorias efetivas nas quais não somente o escalar de curvatura, mas todos os invariantes de curvatura como, por exemplo, $R_{\mu \nu}^{2}, R_{\mu \nu \rho \sigma}^{2}$ e $C_{\mu \nu \rho \sigma}^{2}$, são limitados. Teorias quadráticas são, de fato, bem justificadas, uma vez que cálculos de divergência de um "loop" da relatividade geral $[26,27]$ produzem termos dessa forma na Lagrangeana, além de serem renormalizáveis em todas as ordens [28]. Em geral, sempre podemos 
escrevê-las como

$$
S=\frac{1}{16 \pi G} \int d^{4} x \sqrt{-g}\left[R+\alpha C_{\mu \nu \rho \sigma}^{2}+\beta R_{\mu \nu}^{2}\right],
$$

onde $\alpha$ e $\beta$ são constantes com dimensão de distância ao quadrado. A ausência do quadrado do tensor de Riemann na ação (6.66) se deve ao fato de que, em 4 dimensões, apenas dois termos quadráticos de curvatura (que podem ser escolhidos como sendo $R^{2}$ e $C_{\mu \nu \rho \sigma}^{2}$ ) são independentes [147].

Um problema de importância relacionado com teorias quadráticas diz respeito à presença de graus de liberdade que se propagam com energia cinética negativa, em outras palavras, à presença de campos fantasmas. Podemos entender isso de uma forma simplificada decompondo o propagador do gráviton como [148, 149]

$$
\frac{1}{p^{2}}-\frac{1}{p^{2}-1 / \alpha},
$$

onde o primeiro termo corresponde ao propagador do gráviton usual sem massa, enquanto que o segundo corresponde a um grau de liberdade massivo tal que $m^{2}=1 / \alpha$. O sinal negativo na frente do segundo termo indica que o modo carrega energia negativa.

Potências quadráticas nos invariantes de curvatura emergem genericamente de teorias fundamentais (como, por exemplo, da teoria de cordas - ver, por exemplo, [150]) sob a forma de teorias efetivas. Nesse sentido, podemos argumentar que a ação de Einstein-Hilbert não é exata mesmo no nível clássico, devendo portanto ser corrigida acrescentando-se os dois últimos termos presentes em (6.66) ${ }^{16}$. Nas próximas páginas vamos estudar um modelo cosmológico em uma teoria quadrática na qual todos os invariantes de curvatura são limitados [23, 24, 13], propriedade esta garantida pela hipótese da curvatura limitada [152], segundo a qual se o invariante em questão atingir seu valor limite, então qualquer solução das equações de campo se reduz a uma solução não-singular. Nesse caso, segue que todos os outros invariantes de curvatura serão limitados.

\footnotetext{
${ }^{16}$ Vínculos experimentais sobre as constantes $\alpha$ e $\beta$ são, no entanto, muito fracos devido ao fato de que a curvatura é, usualmente, muito baixa (pelo menos no que se refere a escalas do sistema solar) [151].
} 


\subsubsection{Um modelo não-singular}

Princípios de limitação desempenham um papel chave em física. Em relatividade especial, uma hipótese fundamental é a de que nenhuma partícula pode exceder a velocidade da luz; em mecânica quântica, o princípio da incerteza estabelece um volume mínimo do espaço de fase dentro do qual uma partícula pode ser localizada. No caso da gravitação, a hipótese de curvatura limitada assume a existência de um comprimento fundamental, dado pelo comprimento de Planck $l_{P l}$ (determinado pela constante de Newton $G$ por meio de $\left.l_{P l}=\sqrt{\hbar G / c^{3}}=1,62 \times 10^{-33} \mathrm{~cm}\right)$, tal que não há curvatura correspondendo a escalas $l<l_{P l}$. Por hipótese, portanto, devemos ter:

$$
\begin{aligned}
|R| & \leq l_{P l}^{-2} \\
\left|R_{\mu \nu}^{2}\right| & \leq l_{P l}^{-4} \\
\left|C_{\mu \nu \rho \sigma}^{2}\right| & \leq l_{P l}^{-8}
\end{aligned}
$$

Para realizar o conceito de curvatura limitada, é útil citarmos um exemplo simples extraído de [24] e que utiliza um método baseado em multiplicadores de Lagrange proposto por Altshuler [153]. Seja $A$ a ação de uma partícula clássica de massa $m$ e linha de mundo $x(t)$ :

$$
A=\int d t \frac{1}{2} m \dot{x}^{2}
$$

Com o intuito de limitarmos a velocidade $\dot{x}$, introduzimos em (6.67) o multiplicador de Lagrange $\lambda$ acoplado com $\dot{x}$, e um potencial para $\lambda, V(\lambda)$ :

$$
A_{\text {novo }}=\int d t\left[\frac{1}{2} m \dot{x}^{2}+\lambda \dot{x}^{2}-V(\lambda)\right] .
$$

A equação para $\lambda$ é

$$
\dot{x}^{2}=\partial V / \partial \lambda
$$

mostrando que, contanto que a derivada do potencial seja limitada, $\dot{x}^{2}$ e, portanto, $\dot{x}$, também o serão.

Além de limitarmos $\dot{x}$, também desejamos preservar o limite newtoniano 
da teoria para pequenos $\lambda$ e $\dot{x}$. Nesse limite, o potencial pode ser escrito como

$$
V(\lambda \rightarrow 0) \sim \lambda^{2}+\lambda^{3}+\ldots
$$

(O termo de ordem zero foi escolhido como sendo zero para que no limite de baixas velocidades o potencial não domine sobre o termo cinético.) Uma escolha possível para $V$ é

$$
V(\lambda)=\frac{2 \lambda^{2}}{1+2 \lambda}
$$

Podemos agora eliminar o multiplicador de Lagrange usando sua equação de movimento e exprimindo o potencial em termos da velocidade. Vemos assim que a ação (6.68) se reduz (a menos de uma constante que em nada contribui para a equação de movimento) à ação relativística de uma partícula livre:

$$
A_{\text {novo }}=m \int \sqrt{1-\dot{x}^{2}} d t
$$

Desejamos aplicar o mesmo princípio esboçado acima para o caso gravitacional. Assumindo a hipótese de Penrose [154], segundo a qual o universo iniciou sua evolução de um estado no qual o tensor de Weyl é nulo (o que é o caso para espaços maximalmente simétricos), e com o intuito de implementar a hipótese de curvatura limitada, podemos impor então que, no limite de curvatura máxima, a solução das equações de campo é o espaço-tempo de de Sitter. Em regimes de baixa curvatura, devemos, naturalmente, recuperar o limite da relatividade geral. Genericamente, escrevemos a ação gravitacional como

$$
S_{\text {grav }}=\frac{1}{16 \pi G} \int d^{4} x \sqrt{-g}\left[R+\sum_{i=1}^{N} \varphi_{i} I^{(i)}-V\left(\varphi_{i}\right)\right],
$$

onde $\varphi_{i}(i=1, \ldots, N)$ são os multiplicadores de Lagrange, que dependem das coordenadas do espaço-tempo, e $I^{(i)}$ são funções arbitrárias dos invariantes de curvatura que desejamos limitar:

$$
I^{(i)}=I^{(i)}\left(R, R_{\mu \nu}^{2}, R_{\mu \nu \rho \sigma}^{2}\right)
$$

Para entendermos de que forma podemos construir modelos que contenham limites clássico e de de Sitter em regimes de baixa e alta curvatura, vamos nos restringir ao caso particular $i=1$, ou seja, ao caso de apenas um multiplicador $\varphi_{1} \equiv \varphi$ e apenas um invariante $I^{(1)} \equiv I$. Variando a ação (6.69) em relação a 
$\varphi$, obtemos a seguinte equação de vínculo:

$$
I-\frac{d V}{d \varphi}=0
$$

Em regimes de baixa curvatura impomos que $\varphi$ seja pequeno e que a teoria se reduza à relatividade geral. Portanto, a ação gravitacional pode ser aproximada para

$$
S_{\text {grav }}=\frac{1}{16 \pi G} \int\left[R+\mathcal{O}\left(\varphi, \varphi^{2}, \ldots\right)\right] \sqrt{-g} d^{4} x,
$$

e o potencial, por causa da equação (6.71), deve se comportar como

$$
V(\varphi \rightarrow 0) \sim \varphi^{2}+\varphi^{3}+\ldots
$$

Por outro lado, em regimes de alta curvatura, quando $|\varphi| \gg 1$, o termo de correção na ação de Einstein-Hilbert torna-se importante. O potencial deve então ser escolhido de tal modo que a solução das equações de campo se aproxime da solução de de Sitter. Novamente da equação de vínculo (6.71), vemos que este requisito será satisfeito desde que

$$
\lim _{\varphi \rightarrow \infty} V(\varphi)=2 \Lambda, \quad \lim _{\varphi \rightarrow \infty} I\left(R, R_{\mu \nu}^{2}, \ldots\right)=0
$$

onde $\Lambda$ é uma constante. Portanto, em regimes de alta curvatura, a ação pode ser escrita como

$$
S_{\text {grav }}=\frac{1}{16 \pi G} \int[R-2 \Lambda] \sqrt{-g} d^{4} x .
$$

Recuperamos assim a ação de Einstein-Hilbert com um termo de constante cosmológica oriundo do potencial.

Nas partes seguintes estaremos interessados no caso particular em que o universo, preenchido por um fluido perfeito de poeira e radiação, está originalmente em fase de contração durante a qual a curvatura do espaço-tempo aumenta. Como a curvatura é agora limitada, o universo deve atingir um raio mínimo e se expandir subseqüentemente.

Uma tal cosmologia pode ser realizada por uma escolha conveniente dos invariantes de curvatura. Assumindo a métrica de FRW, dada por

$$
d s^{2}=-d t^{2}+a^{2}(t)\left[\frac{d r^{2}}{1-k r^{2}}+r^{2}\left(d \theta^{2}+\operatorname{sen}^{2} \theta d \phi^{2}\right)\right]
$$




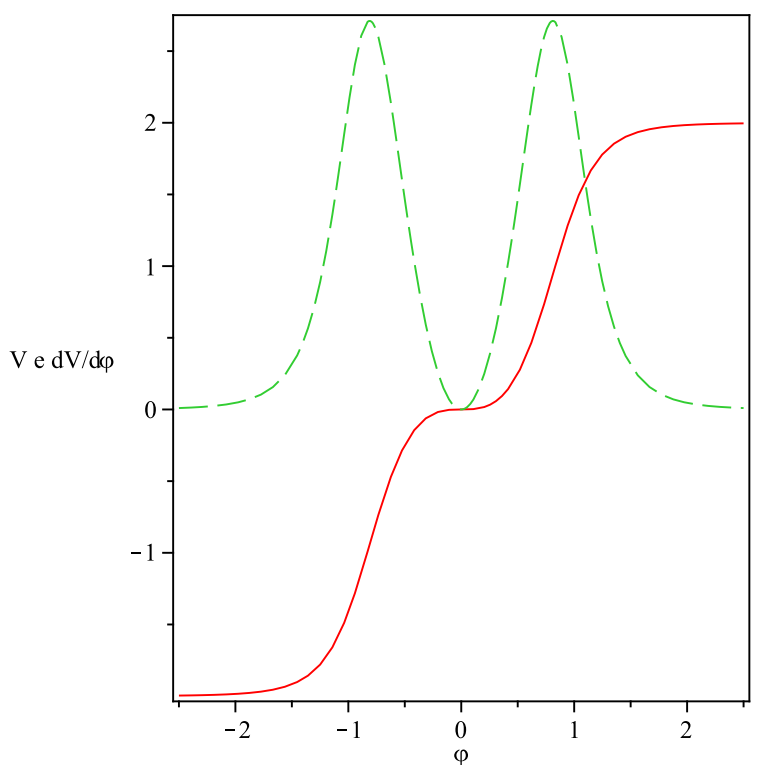

Figura 6.4: Gráfico do potencial $V$ (linha cheia vermelha) e de sua derivada $d V / d \varphi$ (linha tracejada verde).

obtemos equações de segunda ordem livres de singularidades se

$$
I\left(R, R_{\mu \nu}^{2}\right) \equiv R-\sqrt{3\left(4 R_{\mu \nu}^{2}-R^{2}\right)}=12\left(\dot{H}+H^{2}\right)
$$

e com o potencial satisfazendo as condições $V(0)=0, V^{\prime}(0)=0$ e $V( \pm \infty) \rightarrow$ constante.

Um exemplo simples de um potencial satisfazendo as condições acima é

$$
V(\varphi)=\frac{2 \Lambda \varphi^{3}}{\sqrt{1+\varphi^{6}}}
$$

representado na figura (6.4). Substituindo o potencial (6.78) na equação de vínculo (6.71), obtemos

$$
\dot{H}+H^{2}=\frac{1}{12} \frac{d V}{d \varphi}=\frac{\Lambda \varphi^{2}}{2\left(1+\varphi^{6}\right)^{3 / 2}} .
$$

A equação (6.79) nos diz que, quando $H=0, \dot{H}>0$ contanto que a constante cosmológica $\Lambda$ seja positiva. Além disso, para que a fase posterior ao ricochete possa se conectar suavemente com os períodos usuais da cosmologia padrão (para os quais $\dot{H}<0$ ), o parâmetro de Hubble deve atingir um valor máximo, a partir do qual voltará a decrescer. Veremos adiante que uma tal 
evolução cósmica é realizável neste modelo, pelo menos para um certo conjunto de condições iniciais.

Chegamos assim à seguinte ação gravitacional:

$$
S_{\text {grav }}=\frac{1}{16 \pi G} \int\left\{R+\varphi\left[R-\sqrt{3\left(4 R_{\mu \nu}^{2}-R^{2}\right)}\right]-V(\varphi)\right\} \sqrt{-g} d^{4} x
$$

Variando-se a ação (6.80) com relação à métrica obtemos as seguintes equações 17:

$$
\begin{gathered}
R_{\mu \nu}-\frac{1}{2} g_{\mu \nu} R-g_{\mu \nu} \frac{\varphi I}{2}+\varphi I_{R} R_{\mu \nu}-\left(\varphi I_{R}\right)_{; \nu ; \mu} \\
+g_{\mu \nu}\left(\varphi I_{R}\right)_{; \rho}^{; \rho}+2 \varphi I_{P} R_{\mu \rho} R_{\nu}^{\rho}+\left(\varphi I_{P} R_{\mu \nu}\right)_{; \rho}^{; \rho} \\
+g_{\mu \nu}\left(\varphi I_{P} R^{\rho \lambda}\right)_{; \lambda \rho}-2\left(\varphi I_{P} R_{(\mu}^{\lambda} \delta_{\nu)}^{\rho}\right)_{; \rho ; \lambda}+g_{\mu \nu} V(\varphi)=8 \pi G T_{\mu \nu}
\end{gathered}
$$

onde definimos $\partial I / \partial R \equiv I_{R}$ e $\partial I / \partial\left(R_{\mu \nu}^{2}\right) \equiv I_{P}$. Na equação (6.81), também adicionamos uma componente de matéria cujo tensor de momento e energia é $T_{\mu \nu}$.

As equações (6.81) são, em geral, de quarta ordem nas derivadas da métrica. Estão, portanto, sujeitas à instabilidade de Ostrogradsky. Entretanto, no caso particular da métrica maximalmente simétrica de FRW e para a escolha especial do invariante $I$, equação (6.77), a derivada segunda do fator de escala em relação ao tempo cósmico aparece linearmente na Lagrangeana, razão pela qual as equações de movimento serão de segunda ordem no tempo. De fato, a componente $t-t$ das equações (6.81) fica

$$
H^{2}(1+4 \varphi)+2 H \dot{\varphi}+\frac{k}{a^{2}}=\frac{V}{6}+\frac{8 \pi G}{3} \rho
$$

Para as componentes espaciais obtemos

$$
\ddot{\varphi}+4 H \dot{\varphi}+\left(\dot{H}+\frac{3}{2} H^{2}\right)(1+4 \varphi)+\frac{k}{2 a^{2}}=\frac{V}{12}-4 \pi G P .
$$

Como de praxe, $\rho$ e $P$ são a densidade de energia e a pressão do fluido, respectivamente.

Uma questão de importância diz respeito à propagação de perturbações através do ricochete. Uma abordagem clássica para este problema poderia ser bem justificada se considerarmos o fato de que correções de altas energias

\footnotetext{
${ }^{17}$ Os detalhes deste cálculo se encontram no Apêndice III
} 
oriundas de uma teoria fundamental, como, por exemplo, da teoria de cordas, são desprezíveis antes e depois do ricochete. É claro que uma tal aproximação deixa de ser válida na descrição do próprio ricochete. Por essa razão, é de praxe assumir que, em analogia com outras curtas transições do modelo cosmológico padrão, tais como pré-aquecimento [67] e transição entre as eras da radiação e da matéria [65], a duração do ricochete é tal que as perturbações que por ele evoluem preservam seu caráter invariante de escala. Esta hipótese, entretanto, está longe de ser comprovada. Conforme já foi demonstrado em [155], flutuações de grandes comprimentos de onda sofrem efetivamente a influência de uma transição cosmológica deste tipo. Portanto, antes de conhecermos a evolução das flutuações durante um ricochete, é importante entendermos se as equações de campo vinculam (ou não) o tempo característico do ricochete.

Com este propósito em mente, é útil escrevermos as equações de movimento também em termos do tempo conforme $\eta$, definido da maneira usual por $d \eta=d t / a$. Denotando agora por uma linha a derivada em relação ao tempo conforme, a equação de vínculo fica

$$
\mathcal{H}^{\prime}=\frac{a^{2}}{12} \frac{d V}{d \varphi}
$$

onde $\mathcal{H}=a^{\prime} / a$, e a primeira equação de Friedmann modificada fica

$$
\mathcal{H}^{2}(1+4 \varphi)+2 \mathcal{H} \varphi^{\prime}+k=a^{2}\left(\frac{V}{6}+\frac{8 \pi G}{3} \rho\right)
$$

Juntamente com a equação de conservação

$$
\rho^{\prime}+3 \mathcal{H}(\rho+P)=0
$$

as equações acima podem ser combinadas, resultando em

$$
\mathcal{H}^{\prime}(1+4 \varphi)+\varphi^{\prime \prime}+2 \mathcal{H} \varphi^{\prime}=a^{2}\left[\frac{V}{6}-\frac{4 \pi G}{3}(\rho+3 P)\right]
$$

Dadas as equações que descrevem o ricochete, passemos ao seu estudo nas partes que seguem. 


\subsubsection{Ricochete}

Antes de estudarmos os diferentes casos de interesse, é útil definirmos uma nova variável de tempo por $\tau=\sqrt{\Lambda} t$. Redefinindo o parâmetro de Hubble, o fator de escala e a densidade de energia por

$$
\begin{aligned}
H & =\sqrt{\Lambda} h(\tau) \\
\tilde{a} & =\Lambda a \\
\varepsilon(\tau) & =\frac{8 \pi G}{3} \frac{\rho}{\Lambda}
\end{aligned}
$$

obtemos o seguinte sistema de equações adimensionais:

$$
\begin{gathered}
\frac{d \varphi}{d \tau}=\frac{\mathcal{V}}{12 h}-\frac{1}{2} h(1+4 \varphi)-\frac{k}{2 \tilde{a}^{2} h}+\frac{\varepsilon}{2 h} \\
\frac{d h}{d \tau}=\frac{1}{12} \frac{d \mathcal{V}}{d \varphi}-h^{2},
\end{gathered}
$$

onde $\mathcal{V}=V / \Lambda$. A equação de conservação se reduz a

$$
\frac{d \varepsilon}{d \tau}+3 h(\varepsilon+\mathcal{P})=0
$$

onde $\mathcal{P}=8 \pi G P / 3 \Lambda$.

\subsubsection{Dinâmica das equações de campo}

Vamos iniciar esta parte com uma análise dinâmica das equações de movimento no vácuo e sem curvatura espacial. O potencial é dado pela equação (6.78). O que encontramos é que o sistema

$$
\begin{aligned}
\frac{d h}{d \tau} & =\frac{\varphi^{2}}{2\left(1+\varphi^{6}\right)^{3 / 2}}-h^{2} \\
\frac{d \varphi}{d \tau} & =\frac{\varphi^{3}}{6 h \sqrt{1+\varphi^{6}}}-\frac{1}{2} h(1+4 \varphi)
\end{aligned}
$$

tem seis pontos críticos, que correspondem ao número de cruzamentos das curvas definidas por $d h / d \tau=0$ e $d \varphi / d \tau=0$ (ver figura (6.5)). O ponto crítico localizado em $(\varphi, h) \simeq(-0.3,0.21)$ é um ponto assintoticamente estável (um atrator), o que significa que as trajetórias terminam nesse ponto em um tempo $t \rightarrow \infty$, caracterizando assim um atrator de de Sitter. 


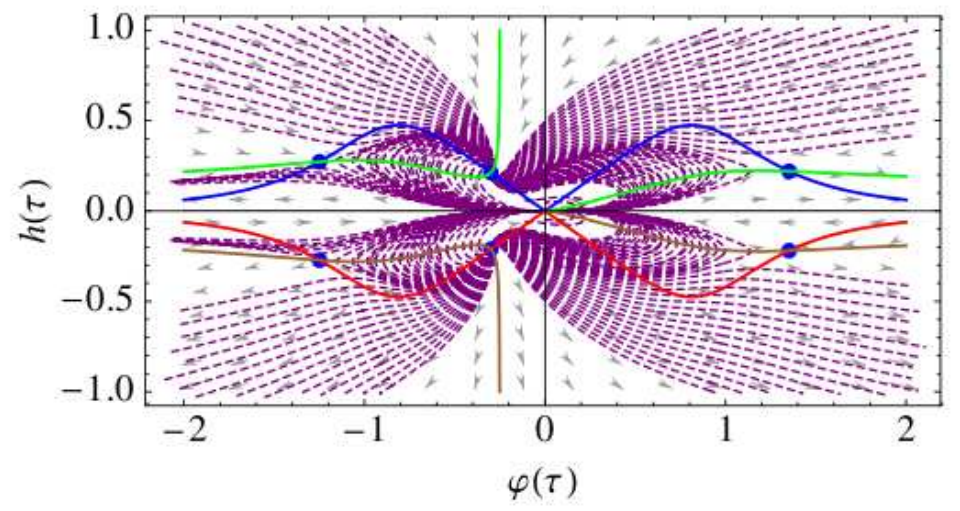

Figura 6.5: Diagrama do espaço de fase no vácuo. Os pontos críticos estão marcados por círculos e localizados nas interseções das curvas $d h / d \tau=0$ e $d \varphi / d \tau=0$. As linhas tracejadas representam algumas soluções particulares do sistema de equações (6.92) e (6.93). As setas indicam a direção do tempo.

O ponto $(\varphi, h) \simeq(-0.3,-0.21)$, por outro lado, é um ponto espiral instável de onde as trajetórias emergem em $t \rightarrow-\infty$. Ele corresponde a um anti-atrator ou, nesse caso, a uma contração de de Sitter. Todos os outros pontos críticos representados na figura (6.5) são pontos de sela, a saber, $(-1.25,-0.27)$, $(-1.25,0.27),(1.35,-0.22)$ e $(\varphi, h) \simeq(1.35,0.22)$. Algumas curvas soluções estão representadas na figura $(6.5)$.

A origem do diagrama do espaço de fase, por sua vez, não é um ponto crítico. Ela corresponde ao ponto de interseção entre os dois ramos da curva $d h / d \tau=0$. De fato, curvas oriundas da região $H<0$ terão necessariamente que passar por ela em um intervalo de tempo finito, caracterizando dessa forma um ponto de convergência em um sistema autônomo. Isto significa que, para as trajetórias que passam por ele, a derivada do campo escalar será descontínua nesse ponto, ou seja, não podemos mais prever o valor de $\dot{\varphi}$ para trajetórias que emergem da origem. Para ver como isto acontece, é útil encontrarmos soluções aproximadas nas vizinhanças de $\varphi \sim 0$ e $H \simeq 0$. Nesse limite, o sistema dinâmico pode ser aproximado para

$$
\frac{d h}{d \tau}=h-\frac{\varphi^{2}}{h}+\mathcal{O}\left(h^{2}, \varphi^{2}, \varphi h\right)
$$

Podemos reescrever a equação acima em termos de $h^{2}$ e integrar a equação diferencial resultante. O resultado final é:

$$
h(\varphi) \simeq \pm \frac{1}{4} \sqrt{A e^{4 \varphi}+1+4 \varphi(1+2 \varphi)},
$$


onde $A$ é uma constante de integração arbitrária. A solução (6.95) obedece à condição de continuidade de Lipschitz na origem, indicando que as trajetórias bidimensionais podem ser vistas como projeções de trajetórias vivendo em um espaço de fase de dimensão maior. Portanto, embora os dois ramos não possam ser suavemente conectados uns aos outros em um diagrama de fase bidimensional, uma abertura desse espaço pela inclusão de dimensões suplementares pode efetivamente curar esta singularidade.

Pontos de sela dividem o diagrama de fase em diferentes regiões através de suas separatrizes (não indicadas na figura (6.5), embora podendo ser inferidas das soluções particulares). Em particular, as separatrizes no terceiro e quarto quadrantes explicam porque há soluções que nunca atingem o eixo $H=0$. Esta é uma propriedade universal, no sentido de que ela não depende do conteúdo de matéria: de fato, isso segue imediatamente da equação de vínculo (6.79), ela mesma independente do conteúdo de matéria. No entanto, do fato do lado direito da equação (6.79) estar acoplada com a densidade via equação (6.82), esperamos um comportamento similar para o parâmetro de Hubble para universos com qualquer tipo de matéria a menos de pequenas diferenças nos detalhes do diagrama de fase.

Vemos assim que ricochetes cosmológicos são bastante gerais nessa classe de modelos de gravidade vinculada, embora as fases de contração e expansão não possam ser conectadas entre si continuamente pelo fato do espaço de fase ser bidimensional. Como veremos nas partes seguintes, a introdução de uma dimensão extra pela introdução ou de curvatura espacial (por menor que ela seja) ou de qualquer tipo de matéria, é o suficiente para regularizar essa tran-

sição, de tal maneira que toda quantidade física do sistema permanecerá finita e livre de singularidades.

\subsubsection{Curvatura espacial}

Por inspeção da figura (6.5), vemos que para toda trajetória na região $h<0$, há uma trajetória similar em $h>0$. O fato problemático é que todas as soluções cruzam a reta $h=0$ através da origem, o que, para o sistema dinâmico autônomo bidimensional estudado na parte precedente, não faz sentido. Vejamos como a introdução de curvatura espacial pode resolver esse problema.

De fato, mesmo com uma quantidade ínfima de curvatura, trajetórias oriundas do quarto quadrante $(\varphi>0, h<0, d \varphi / d \tau<0, d h / d \tau \gtrsim 0)$ emergem no 
segundo quadrante $(\varphi<0, h>0, d \varphi / d \tau<0, d h / d \tau \gtrsim 0)$, ao passo que trajetórias oriundas do terceiro quadrante emergem no primeiro quadrante, de tal modo que as soluções particulares das equações de movimento são perfeitamente contínuas. Isto só ocorre porque agora o fator de escala e o parâmetro de Hubble são vistos como variáveis dinâmicas independentes, ou seja, o espaço de fase que antes era bidimensional, passa a ser tridimensional com a presença da curvatura espacial, permitindo desse modo que as trajetórias não mais se cruzem.

Para ver isso, consideremos o sistema dinâmico composto pelas equações (6.84) - (6.86). A equação problemática que tornou o cenário anterior intrincado é a equação (6.85). Tomando-se o limite $\mathcal{H} \rightarrow 0$, esta equação se reduz a

$$
\varphi^{\prime} \simeq \frac{1}{2 \mathcal{H}}\left(\frac{a^{2} V}{6}-k\right)
$$

A taxa de variação do fator de escala é obviamente nula no ricochete $(\mathcal{H} \rightarrow 0)$. Logo, se a derivada do campo acima é finita, então $\varphi \rightarrow \varphi_{b}$ e $a \rightarrow a_{b}$ no ricochete. Isto significa que $V\left(\varphi_{b}\right)=6 k / a^{2}$, o que, por sua vez, implica, juntamente com a definição do potencial, equação (6.78), que

$$
\varphi_{b}^{6}=\left(\frac{\Lambda^{2} a_{b}^{4}}{9 k^{2}}-1\right)^{-1}
$$

É claro que, quando $k \rightarrow 0^{ \pm}, \varphi_{b} \rightarrow 0$, ou seja, o sistema físico é perfeitamente bem comportado para qualquer valor não-nulo da curvatura espacial.

Portanto, concluímos que é possível conectar os dois ramos da teoria continuamente desde que haja uma quantidade qualquer, tão pequena quanto se queira, de curvatura. Como o limite de curvatura nula é ele mesmo bem comportado, concluímos também que, contanto que a dimensão extra do espaço de fase seja preservada, a conexão entre os dois ramos é realizável também na ausência de curvatura. A construção de modelos de ricochete com curvatura nula fica assim estabelecida.

É interessante notar que a condição (6.97) implica uma curvatura espacial máxima $k_{\max }$ dada por

$$
\left|k_{\max }\right|=\frac{\Lambda a_{b}^{2}}{3}=H_{\Lambda}^{2} a_{b}^{2},
$$

o que é na verdade perfeitamente legítimo, uma vez que estamos limitando a 
curvatura do espaço-tempo.

Estas considerações estão ilustradas na figura (6.6), onde estão representadas a evolução temporal do fator de escala, do parâmetro de Hubble reduzido e do campo escalar para três cenários com diferentes valores para a curvatura espacial.

\subsubsection{Gravidade vinculada com fluido de matéria}

Tendo estabelecido que a curvatura espacial pode ser completamente ignorada, vamos continuar com a análise das equações de campo para o caso plano, mas desta vez na presença de matéria. Nesse caso, as equações dinâmicas são dadas por

$$
\begin{gathered}
\frac{d h}{d \tau}=-h^{2}+\frac{\varphi^{2}}{2\left(1+\varphi^{6}\right)^{3 / 2}} \\
\frac{d \varphi}{d \tau}=-\frac{1}{2} h(1+4 \varphi)+\frac{\varphi^{3}}{6 h \sqrt{1+\varphi^{6}}}+\frac{\varepsilon}{2 h} \\
\frac{d \varepsilon}{d \tau}=-3 h(1+w) \varepsilon
\end{gathered}
$$

Vamos nos concentrar sobre uma classe de soluções do sistema de equações (6.99) - (6.101) que nos dá informação sobre como a matéria afeta o ricochete. Com esse propósito, voltemos ao caso bidimensional, definido pelas equações (6.92) e (6.93). Vimos que, nesse caso, não apenas $h \rightarrow 0$ perto do ricochete, mas também que $d h / d \tau$ e $d \varphi / d \tau$ se anulam nessa região. A evolução através do ricochete é então dominada pelos termos de derivada superior em $h$ e em $\varphi$, que podem ser ajustados por uma escolha conveniente das condições iniciais de tal modo que $h \rightarrow 0$ durante um longo intervalo de tempo. Em outras palavras, dependendo das condições iniciais do universo, o espaço-tempo pode permanecer em um estado "quase-Minkowski" ( $h \simeq 0$ ) durante um longo período de tempo cósmico antes de entrar em uma fase de super-aceleração com $d h / d \tau>0$. Na presença de matéria, entretanto, o que verificamos é que o universo inicia sua evolução em uma fase de contração de de Sitter e passa rapidamente pelo ricochete, emergindo em um período de super-inflação antes de atingir o atrator final de de Sitter. É, portanto, bastante similar ao caso anterior com curvatura espacial. Uma trajetória particular para o caso com matéria está ilustrada na figura (6.7). Note que, para o caso com matéria, não há um plano de fase, uma vez que o espaço de fase tem agora três dimensões. 

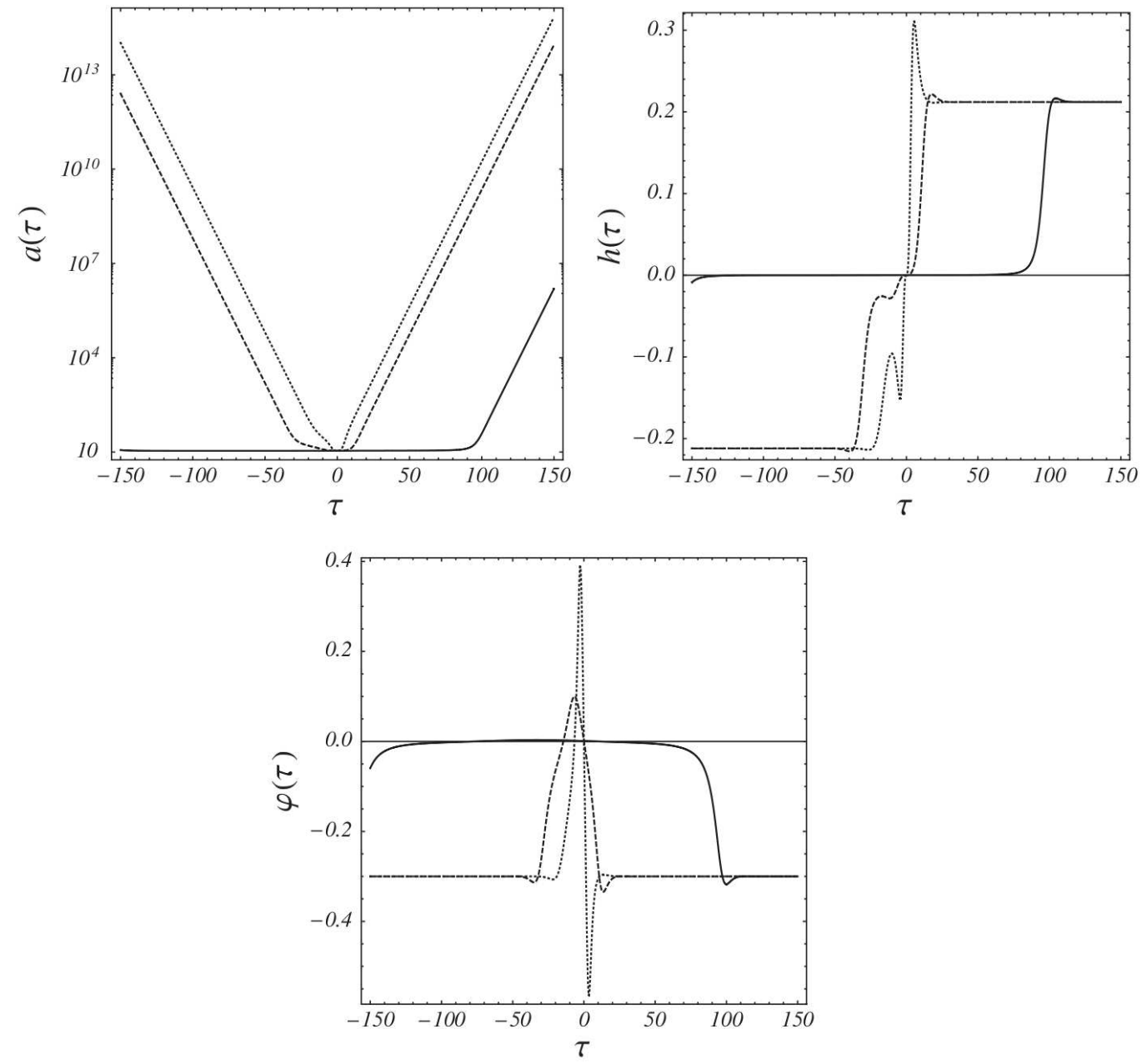

Figura 6.6: Evolução temporal do fator de escala (no alto à esquerda), do parâmetro de Hubble (no alto à direita) e do campo escalar (em baixo). As diferentes linhas correspondem cada uma a uma quantidade diferente de curvatura: para $k$ muito pequeno $\left(k=10^{-9}\right.$, linha contínua) e para $k$ pequeno $\left(k=10^{-3}\right.$, linha tracejada, e $k=10^{-2}$, linha pontilhada). O caso com $k$ muito pequeno é interessante: o universo passa por um estado com o fator de escala constante durante um longo período de tempo, depois do qual entra em expansão espontaneamente. A evolução na direção passada indica que o intervalo de tempo passado durante o qual $h \rightarrow 0$ só é possível quando $k=0$. Do contrário, o estado de Minkowski sempre se origina de uma fase de contração de de Sitter. 


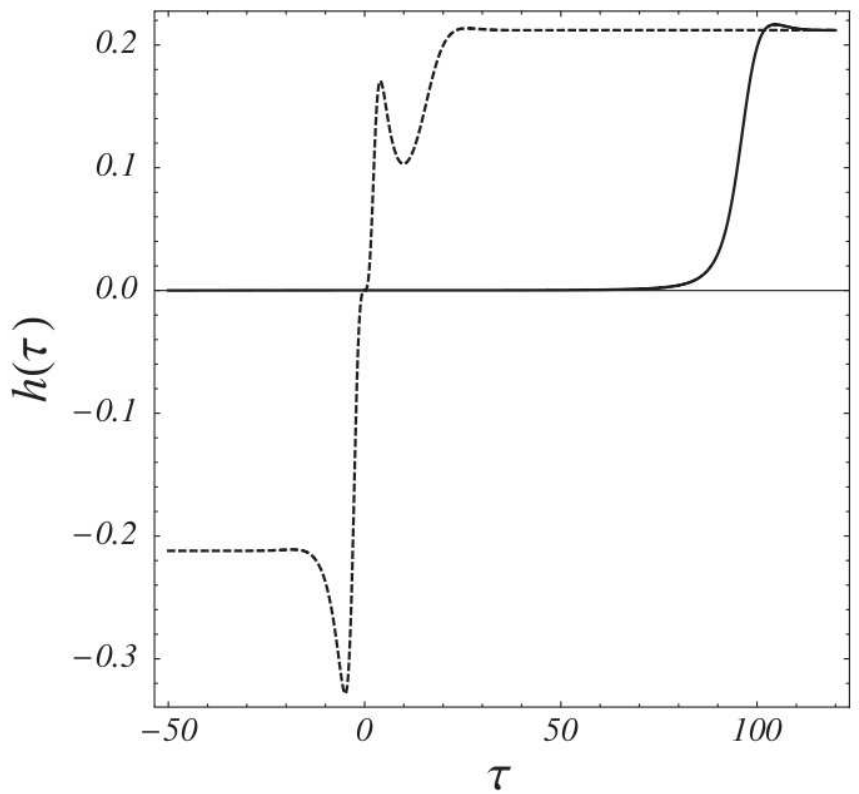

Figura 6.7: Evolução temporal do parâmetro de Hubble no vácuo (linha contínua) e com matéria (linha tracejada). Quando uma pequena quantidade de matéria é adicionada ao sistema $\left(\rho_{\text {rad }}(0)=10^{-5}\right.$ neste exemplo) o fator de escala evolui de uma contração de de Sitter ao ricochete. A fase final corresponde ao eterno período de expansão acelerada de de Sitter. Os resultados são exatamente similares para o caso com poeira $(w=0)$ ou com fluido do tipo curvatura $(w=-1 / 3)$. Os três casos estão ilustrados na figura (6.8). É importante ressaltar o fato de que a presença de matéria diminui drasticamente a duração do ricochete, de tal modo que a existência de um modelo de universo emergente é, nesse caso, bastante improvável. 

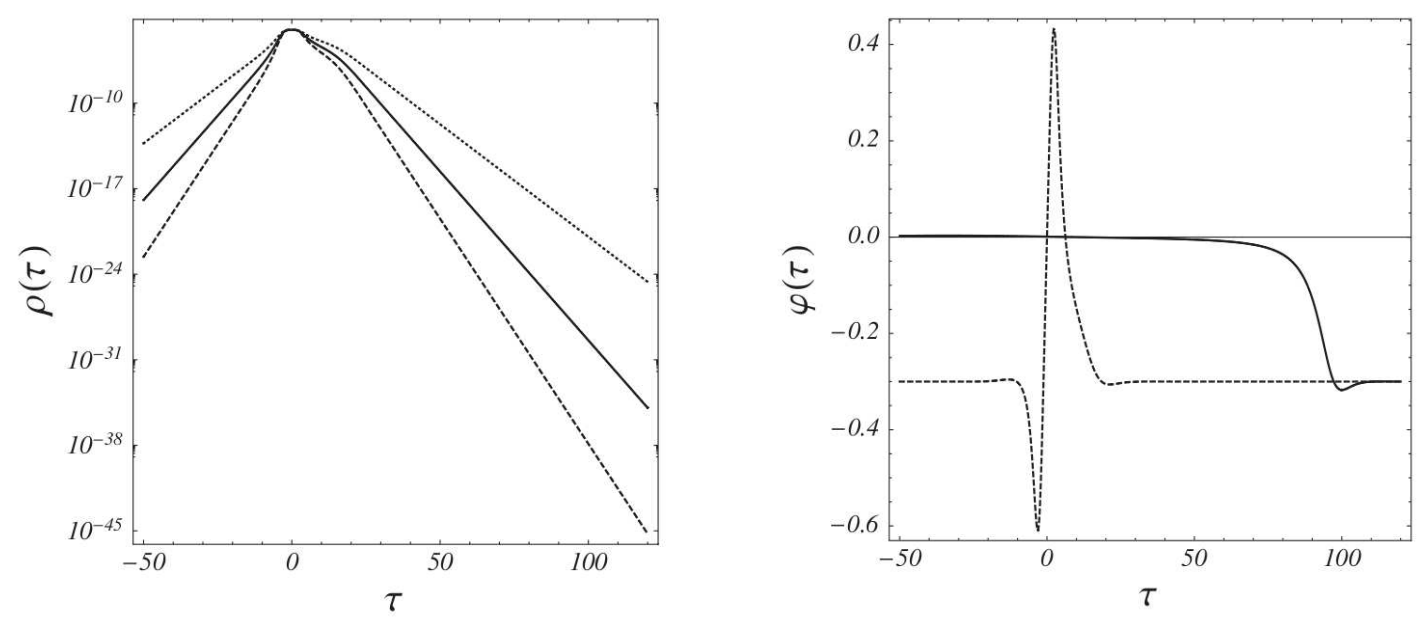

Figura 6.8: À esquerda, temos a evolução das densidades quando o fluido consiste de poeira (linha contínua), radiação (linha tracejada) e fluido do tipo curvatura (linha pontilhada). À direita, temos a evolução do campo escalar no vácuo (linha contínua) e com matéria (linha tracejada). Este último caso inclui ou poeira, ou radiação (as linhas são indistingüíveis).

As possíveis trajetórias projetadas sobre o plano bidimensional são essencialmente as mesmas que aquelas obtidas para o sistema bidimensional (figura $(6.5))$.

Portanto, em uma teoria de gravidade vinculada, é sempre possível construirmos modelos cosmológicos homogêneos e isotrópicos, espacialmente planos e com um fluido de poeira e radiação que se comporta da seguinte maneira: o universo inicia sua evolução durante uma fase de contração. O fator de escala atinge um mínimo, passa por um ricochete regular e começa a se expandir de modo super-acelerado. Quando o parâmetro de Hubble atinge um certo valor máximo (que depende dos detalhes dos vínculos do espaço-tempo), ele passa a decrescer de modo similar ao caso de um universo de FRW, até atingir seja uma fase de de Sitter, seja um outro regime como estado final.

Os comportamentos discutidos acima são universais, no sentido de que não dependem crucialmente da forma do potencial $V(\varphi)$. A título de exemplo, a figura (6.9) representa o diagrama de fase para o seguinte potencial:

$$
V_{2}(\varphi)=\frac{2 \Lambda \varphi}{\sqrt{1+\varphi^{6}}}
$$




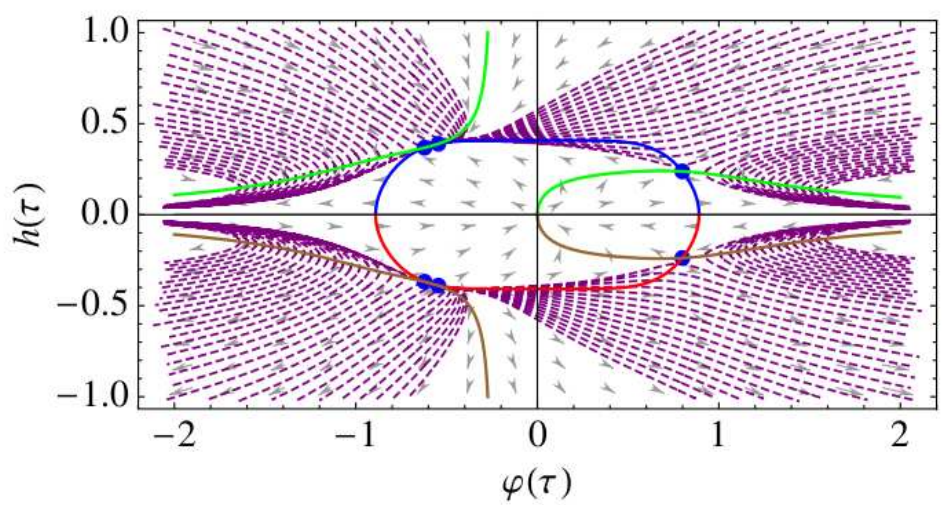

Figura 6.9: Diagrama de fase para o potencial $V_{2}(\varphi)$ dado pela equação $(6.102)$ e representado na figura (6.10).

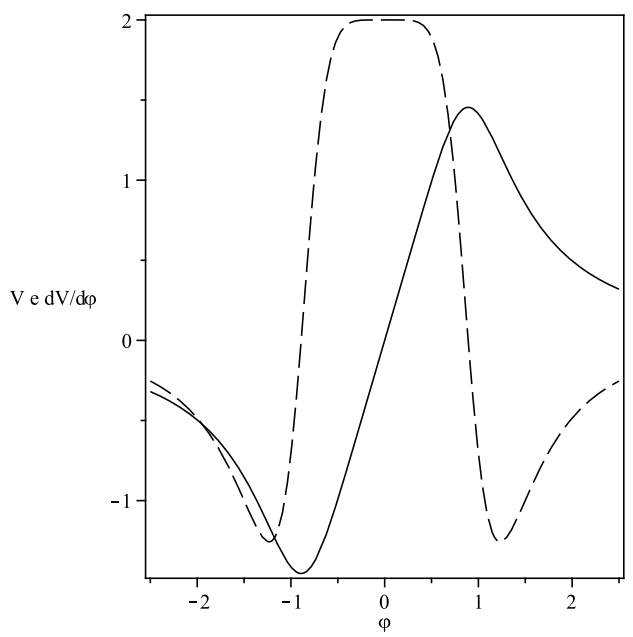

Figura 6.10: Potencial $V_{2}(\varphi)$ (linha contínua) e sua derivada (linha descontínua). 


\subsubsection{Escala de tempo típica do ricochete}

Nesta parte desejamos calcular o tempo característico do ricochete descrito pelo modelo acima. Mais especificamente, mostraremos que, em contraste com a descrição clássica da relatividade geral [11, 21], nesta classe de teorias a escala de tempo típica do ricochete pode ser tão pequena quanto se queira.

Vamos começar com a seguinte expansão para o fator de escala [11] em torno do ricochete:

$$
a(\eta)=a_{0}\left[1+\frac{1}{2}\left(\frac{\eta}{\eta_{0}}\right)^{2}+\delta\left(\frac{\eta}{\eta_{0}}\right)^{3}+\frac{5}{24}(1+\xi)\left(\frac{\eta}{\eta_{0}}\right)^{4}\right]
$$

onde escolhemos o instante do ricochete como sendo em $\eta=0$. Na expansão acima, $\eta_{0}$ define o tempo característico do ricochete. Qualquer outro objeto portando o rótulo 0 tem seu valor calculado em $\eta=0$. Conforme está explicado na referência [11], os parâmetros $\xi$ e $\delta$ controlam a amplitude do desvio em relação a um ricochete de de Sitter, cujo fator de escala é $a(\eta)=a_{0} \sqrt{1+\tan ^{2}\left(\eta / \eta_{0}\right)}$, explicando assim o número 5/24 diante do termo de quarta ordem da expansão (6.103).

De um modo similar, assumimos que o campo escalar $\varphi(\eta)$, seu potencial $V(\eta)$ e a densidade de energia $\rho(\eta)$ também admitem uma expansão em série de Taylor em torno de $\eta=0$. Explicitamente, escrevemos

$$
\begin{aligned}
& \varphi(\eta)=\varphi_{0}+\varphi_{1}\left(\frac{\eta}{\eta_{0}}\right)+\varphi_{2}\left(\frac{\eta}{\eta_{0}}\right)^{2}+\varphi_{3}\left(\frac{\eta}{\eta_{0}}\right)^{3}+\ldots, \\
& V(\eta)=V_{0}+V_{1}\left(\frac{\eta}{\eta_{0}}\right)+V_{2}\left(\frac{\eta}{\eta_{0}}\right)^{2}+V_{3}\left(\frac{\eta}{\eta_{0}}\right)^{3}+\ldots, \\
& \rho(\eta)=\rho_{0}+\rho_{1}\left(\frac{\eta}{\eta_{0}}\right)+\rho_{2}\left(\frac{\eta}{\eta_{0}}\right)^{2}+\rho_{3}\left(\frac{\eta}{\eta_{0}}\right)^{3}+\ldots,
\end{aligned}
$$

onde $\varphi_{i}, V_{i}$ e $\rho_{i}$ são parâmetros a serem determinados à seguir.

Para determinarmos os coeficientes das expansões acima e, por consegüinte, o tempo característico do ricochete, basta verificarmos de que modo eles estão vinculados pelas equações de campo. Na prática, inserimos a expansão (6.103) nas equações (6.84) - (6.86) e identificamos os termos ordem a ordem em $\eta / \eta_{0} \ll 1$. Obtemos assim

$$
\rho_{1}=0
$$




$$
\begin{aligned}
\rho_{2} & =-\frac{3}{2}(1+w) \rho_{0} \\
\rho_{3} & =-3 \delta(1+w) \rho_{0}
\end{aligned}
$$

Note que a condição de ricochete simétrico $(\delta=0)$ implica, como esperado, $\rho_{3}=0$, ou seja, a densidade de energia é uma função par do tempo conforme. A primeira igualdade, equação (6.107), expressa simplesmente a conservação da energia em $\eta=0$.

A duração do ricochete em termos do tempo cósmico pode então ser calculada como sendo

$$
t_{0}^{2} \equiv a_{0}^{2} \eta_{0}^{2}=\frac{12 \varphi_{1}}{V_{1}}=12\left(\left.\frac{d V}{d \varphi}\right|_{0}\right)^{-1}
$$

onde o último termo é a derivada do potencial em relação a $\varphi$ calculada em $\eta=0$. Este termo permanece como um parâmetro livre, como podemos ver por inspeção das relações seguintes para o potencial:

$$
\begin{gathered}
V_{0}=\frac{6 k}{a_{0}^{2}}-16 \pi G \rho_{0}, \\
V_{2}=\left.\frac{d V}{d \varphi}\right|_{0}\left(3 \delta \varphi_{1}+\varphi_{2}\right),
\end{gathered}
$$

Note que, para curvatura espacial nula ou negativa, o potencial é negativo no ricochete, de tal modo que, como no caso da relatividade geral, um simples campo escalar massivo não pode levar o universo a passar por uma fase de ricochete a menos que a curvatura seja positiva.

Em ordens superiores obtemos relações entre os termos de derivada superior do campo escalar no ricochete e a densidade de energia e o parâmetro de assimetria do fator de escala naquele ponto. Mais precisamente, obtemos

$$
\begin{gathered}
\varphi_{2}=-\frac{1}{2}\left(1+4 \varphi_{0}\right)+6\left(\left.\frac{d V}{d \varphi}\right|_{0}\right)^{-1}\left[\frac{k}{a_{0}^{2}}-4 \pi G \rho_{0}(1+w)\right], \\
\varphi_{3}=-\delta\left(1+4 \varphi_{0}\right)-\frac{2}{3} \varphi_{1} .
\end{gathered}
$$

O coeficiente de quarta ordem do fator de escala, por sua vez, é dado por

$$
\xi=\frac{6}{5}\left\{\frac{1}{V_{1}}\left[V_{3}+24 \pi G \delta \rho_{0}(1+w)-24 \delta \frac{k}{a_{0}^{2}}\right]+\frac{2 \delta\left(1+4 \varphi_{0}\right)-\varphi_{3}}{\varphi_{1}}\right\} .
$$


Uma importante conclusão que diz respeito às relações acima é que a escala típica do ricochete pode ser arbitrariamente pequena. Vemos que, para um dado $a_{0}$, quanto maior for a derivada do potencial no ricochete ou, posto de outra forma, quanto maior for a constante $\sqrt{\Lambda}$ que define a dimensão de $V$, menor será a escala de tempo do ricochete. Este resultado pode ser consistentemente verificado por integração numérica do sistema (6.85): consideremos as equações no vácuo, equações (6.92) e (6.93). No ponto $(\varphi=0, H=0)$, a derivada do potencial é exatamente zero, o que significa, de acordo com a relação (6.110), que a escala de tempo físico do ricochete vai a infinito. Em outras palavras, para o caso de um universo em contração na ausência de matéria, não há transição entre a fase original e a fase de expansão.

\subsubsection{Comentários}

Em relatividade geral, construir modelos de ricochete em um universo preenchido por fluidos bem comportados de matéria é uma tarefa altamente nãotrivial: tipicamente, devemos ter violação da condição de energia forte, além da curvatura espacial ser estritamente positiva, fato este que vai de encontro com as observações. Portanto, ao menos que se realize uma fase de inflação após o ricochete ${ }^{18}$, é necessário alterar seja o conteúdo de matéria, seja a teoria da gravidade.

A maneira mais simples de se construir modelos de ricochetes regulares em um universo com um fluido de matéria bem comportado é assumindo que a seção espacial tenha curvatura positiva, permitindo desta forma que o parâmetro de Hubble se anule no ricochete. Nesse caso, o que se encontra é que o tempo característico dessa transição é limitado inferiormente, de tal modo que a ocorrência de um ricochete arbitrariamente curto está completamente excluída.

Nesse trabalho investigamos um ricochete não-singular no âmbito de uma teoria de gravidade modificada proposta em [24]. Encontramos soluções que correspondem a um universo em fase de contração seguida de super-inflação, antes de se conectar suavemente a um período de expansão desacelerada, como ocorre na cosmologia padrão. A fase final emerge, por sua vez, como um universo de de Sitter. Calculamos também a duração do ricochete para esse modelo. Encontramos que, qualquer que seja o ricochete, isto é, se ocorre em

\footnotetext{
${ }^{18} \mathrm{Ou}$ que a fase de contração seja bem mais longa que a fase de expansão - ver seção 2.4 .
} 
um universo plano, aberto ou fechado, se é ou não é simétrico, e se ocorre na presença ou na ausência de matéria, o seu tempo característico, em oposição ao seu análogo clássico, é completamente arbitrário. Este fato poderia ter conseqüências importantes sobre a propagação de flutuações que evoluem através do ricochete. Nesse sentido, embora para a nossa escolha da Lagrangeana as equações de movimento de fundo sejam de segunda ordem no tempo, o setor perturbativo provavelmente exibirá sinais da instabilidade inerente a teorias a derivadas mais altas, de tal modo que, até o presente momento, não está claro se as perturbações no âmbito desses modelos podem ou não ser tratadas judiciosamente. 


\section{Capítulo 7}

\section{Conclusões e perspectivas}

Além de seus próprios sucessos como teoria da gravidade, a relatividade geral, juntamente com o princípio da homogeneidade e isotropia, oferece uma descrição judiciosa de nosso universo consistente com as observações. Entre os sucessos do modelo cosmológico padrão, podemos citar sua previsão segundo a qual o universo é preenchido por uma radiação de fundo com espectro de corpo negro e a previsão da abundância relativa de elementos leves. Mais recentemente, com a descoberta de que o universo está em expansão acelerada, uma explicação no âmbito desse modelo mostrou-se efetiva: o estado atual do universo é perfeitamente compatível com a hipótese de que seu principal conteúdo material é descrito por uma energia de vácuo constante, ou seja, por uma constante cosmológica.

Esta hipótese, porém, é discutível. No que diz respeito à introdução de contribuições escuras para a densidade de energia do universo, não sabemos explicar a razão pela qual a constante cosmológica é tão pequena, ou por que as densidades de matéria e energia escuras são da mesma ordem de grandeza. São questões importantes que motivam a investigação de modelos alternativos, um deles no contexto de teorias de gravidade modificada.

Modificar a relatividade geral, no entanto, está longe de ser uma tarefa trivial. É preciso levar em consideração todas as previsões de uma teoria robusta consistente com todos os testes realizados até o presente momento. Entre eles, há os testes de sistema solar, assim como os testes do modelo cosmológico padrão. Considerações teóricas como, por exemplo, problemas de estabilidade também devem ser levados em conta.

Um exemplo de teoria de gravidade generalizada são as teorias $f(R)$, ou 
escalar-tensoriais. A simplicidade desta classe de teorias (simples no sentido de que constituem uma generalização imediata da ação de Einstein-Hilbert), no entanto, não se reflete nos vínculos que devem ser satisfeitos para que possam, de fato, ser consideradas como candidatas a uma boa teoria gravitacional: para que vínculos de sistema solar e cosmológicos sejam satisfeitos simultaneamente, é necessário criar um mecanismo especial, conhecido como mecanismo do camaleão, que confere à partícula escalar uma massa variável dependente da densidade local de energia. Esta massa, porém, deixa de ser bem comportada em épocas primordiais, indicando a existência de uma singularidade já na era dominada pela matéria. Como vimos neste trabalho, uma forma de se resolver este problema é no âmbito de ricochetes cosmológicos. A questão que persiste é como se poderia acomodar uma transição deste tipo em um modelo completo consistente com todos os vínculos.

Voltando à esfera do modelo cosmológico padrão, além do problema da constante cosmológica, há os problemas de como explicar a planura do universo e a isotropia da radiação cósmica de fundo, assim como a questão da origem de flutuações de densidade responsáveis pela formação de estruturas em largas escalas. São, em suas essências, problemas relacionados com a determinação de condições iniciais específicas. Este é o papel, por exemplo, da teoria inflacionária, que, além de esclarecer os problemas intrínsecos ao modelo cosmológico padrão, também prevê um espectro de perturbações escalares aproximadamente invariante de escala.

O paradigma inflacionário encontra ainda uma motivação teórica ao poder ser implementado em teorias de física de partículas de altas energias. Porém, ainda que bem motivada e fenomenologicamente bem sucedida, não é a única possibilidade que delas emerge. Podemos, por exemplo, considerar outros modelos oriundos da compactificação de dimensões extras em teoria $\mathrm{M}$ ou teoria de cordas. Exemplos são as cosmologias "ekpiróticas" e pré-big bang, ambas exibindo a característica em comum de apresentarem fases de contração seguida de expansão, ou seja, de apresentarem ricochetes. Transições desse tipo, além de resolverem os problemas do horizonte e da planura, podem ser não-singulares, abordando assim uma questão deixada em aberto no âmbito da teoria inflacionária.

A regularização de ricochetes não é, por sua vez, uma tarefa trivial. Uma forma de fazê-lo é por meio de termos de ordem mais alta nos invariantes de curvatura. Neste trabalho, investigamos uma teoria efetiva particular em um 
espaço-tempo homogêneo e isotrópico no qual todos os invariantes de curvatura são limitados. Obtivemos uma cosmologia diversificada, que exibe fases de contração e expansão livres de singularidade, na ausência e na presença de curvatura espacial e com fluidos hidrodinâmicos usuais. Em particular, para um certo conjunto de condições iniciais, o universo inicia sua evolução em um período de contração até atingir um raio mínimo. A expansão subseqüente é caracterizada por um período de superinflação, durante o qual o raio de Hubble cresce até atingir um valor máximo, voltando a decrescer como no universo de Friedmann. O período final emerge como uma fase de expansão de de Sitter. O ricochete, por sua vez, além de ser regular, tem uma duração arbitrária, contrariamente ao que ocorre nos modelos usuais, com curvatura. Esta propriedade poderia ter importantes conseqüências no que se refere à propagação de flutuações através do ricochete.

Em relação a este último aspecto, é importante notar que, por ser uma teoria a derivadas mais altas, está assim sujeita à instabilidade de Ostrogradski. No entanto, justamente por ser efetiva, é vista como uma aproximação de baixas energias de uma teoria fundamental exata, livre de singularidades e de instabilidades. Logo, se a aproximação que consideramos está correta, devemos esperar que, em um certo regime, a solução aproximada corresponderá efetivamente à solução exata, ou seja, a uma solução estável e não-singular. A determinação das soluções estáveis em um regime apropriado constitui uma importante questão que merece ainda ser investigada. 


\section{Apêndice I}

Vamos determinar as equações de movimento que seguem da seguinte ação:

$$
S=\frac{1}{16 \pi G} \int f(R) \sqrt{-g} d^{4} x
$$

Para esse propósito, a seguinte identidade pode ser útil:

$$
A_{; \mu}^{\mu}=\frac{1}{\sqrt{-g}} \frac{\partial}{\partial x^{\mu}}\left(\sqrt{-g} A^{\mu}\right)
$$

Variando então (7.1) com respeito à métrica, obtemos:

$$
\begin{aligned}
\delta S & =\frac{1}{16 \pi G} \int d^{4} x\left\{f \delta \sqrt{-g}+\sqrt{-g} f^{\prime} \delta R\right\} \\
\delta \sqrt{-g} & =-\frac{\sqrt{-g}}{2} g_{\mu \nu} \delta g^{\mu \nu} \\
\delta R & =\delta g^{\mu \nu} R_{\mu \nu}+g_{\mu \nu} \delta R_{\mu \nu} \\
\delta S & =\frac{1}{16 \pi G} \int d^{4} x\left\{\frac{-f}{2} \sqrt{-g} g_{\mu \nu} \delta g^{\mu \nu}+\sqrt{-g} f^{\prime}\left(\delta g^{\mu \nu} R_{\mu \nu}+g_{\mu \nu} \delta R_{\mu \nu}\right)\right\}
\end{aligned}
$$

Vamos calcular $\delta R_{\mu \nu}$ em um referencial localmente inercial antes de integrarmos o último termo de $\delta S$ por partes. Nesse referencial as primeiras derivadas da métrica se anulam e derivadas covariantes se transformam em derivadas parciais. Assim:

$$
\begin{aligned}
\delta R_{\mu \nu} & =\frac{\partial}{\partial x^{\lambda}} \delta \Gamma_{\mu \nu}^{\lambda}-\frac{\partial}{\partial x^{\nu}} \delta \Gamma_{\mu \lambda}^{\lambda} \\
\delta \Gamma_{\mu \nu}^{\lambda} & =\frac{1}{2} g^{\lambda \rho}\left(\delta g_{\rho \mu, \nu}+\delta g_{\rho \nu, \mu}-\delta g_{\mu \nu, \rho}\right) \\
\sqrt{-g} g^{\mu \nu} \delta R_{\mu \nu} & =\frac{\partial}{\partial x^{\lambda}}\left[\left(g^{\mu \nu} \delta \Gamma_{\mu \nu}^{\lambda}-g^{\mu \lambda} \delta \Gamma_{\mu \nu}^{\nu}\right) \sqrt{-g}\right]
\end{aligned}
$$

Na última linha do lado direito há a derivada do vetor $\left(g^{\mu \nu} \delta \Gamma_{\mu \nu}^{\lambda}-g^{\mu \lambda} \delta \Gamma_{\mu \nu}^{\nu}\right) \sqrt{-g} \equiv$ ()$^{\lambda}$, que expressamos em um referencial não-lorenziano arbitrário pela subs- 
tituição $\frac{\partial}{\partial x^{\lambda}} \rightarrow \nabla_{\lambda}$. Usando a identidade (7.2), temos:

$$
\begin{aligned}
\frac{\partial}{\partial x^{\lambda}}\left[\left(g^{\mu \nu} \delta \Gamma_{\mu \nu}^{\lambda}-g^{\mu \lambda} \delta \Gamma_{\mu \nu}^{\nu}\right) \sqrt{-g}\right] & =\nabla_{\lambda}\left[\sqrt{-g}()^{\lambda}\right] \\
& =\frac{1}{\sqrt{-g}} \frac{\partial}{\partial x^{\lambda}}\left[\sqrt{-g}()^{\lambda}\right] \\
\int d^{4} x \sqrt{-g} f^{\prime} g^{\mu \nu} \delta R_{\mu \nu} & =\int d^{4} x f^{\prime} \frac{\partial}{\partial x^{\lambda}}\left[\sqrt{-g}()^{\lambda}\right] \\
& =\sqrt{-g} f^{\prime}()^{\lambda}-\int d^{4} x \sqrt{-g}()^{\lambda} \frac{\partial}{\partial x^{\lambda}} f^{\prime} \\
= & 0-\int d^{4} x \sqrt{-g}()^{\lambda} \frac{\partial}{\partial x^{\lambda}} f^{\prime} \\
-\int d^{4} x \sqrt{-g}()^{\lambda} \frac{\partial}{\partial x^{\lambda}} f^{\prime}= & -\int d^{4} x \sqrt{-g} g^{\mu \nu} \delta \Gamma_{\mu \nu}^{\lambda} f_{, \lambda}^{\prime} \\
& +\int d^{4} x \sqrt{-g} g^{\mu \lambda} \delta \Gamma_{\mu \nu}^{\nu} f_{, \lambda}^{\prime}
\end{aligned}
$$

Para calcular as integrais da última linha, vamos usar o mesmo truque, ou seja, calcular os integrandos em um referencial localmente inercial antes de efetuarmos a integração por partes em um referencial arbitrário. Lembremos antes que

$$
\delta g^{\mu \nu}=-g^{\mu \rho} g^{\nu \sigma} \delta g_{\rho \sigma}
$$

Para o primeiro integrando, obtemos:

$$
\begin{aligned}
\sqrt{-g} g^{\mu \nu} \delta \Gamma_{\mu \nu}^{\lambda} f_{, \lambda}^{\prime}= & \frac{\sqrt{-g} g^{\mu \nu}}{2} f_{, \lambda}^{\prime} g^{\lambda \rho}\left(\delta g_{\rho \mu, \nu}+\delta g_{\rho \nu, \mu}-\delta g_{\mu \nu, \rho}\right) \\
= & \frac{\sqrt{-g} g^{\mu \nu}}{2} f_{, \lambda}^{\prime} g^{\lambda \rho}\left[\frac{\partial}{\partial x^{\nu}}\left(f_{, \lambda}^{\prime} \delta g_{\rho \mu}\right)-f_{, \lambda \nu}^{\prime} \delta g_{\rho \mu}+\frac{\partial}{\partial x^{\mu}}\left(f_{, \lambda}^{\prime} \delta g_{\rho \nu}\right)\right. \\
& \left.-f_{, \lambda \mu}^{\prime} \delta g_{\rho \nu}-\frac{\partial}{\partial x^{\rho}}\left(f_{, \lambda}^{\prime} \delta g_{\mu \nu}\right)+f_{, \lambda \rho}^{\prime} \delta g_{\mu \nu}\right] \\
= & \sqrt{-g} \frac{\partial}{\partial x^{\nu}}\left[g^{\mu \nu} g^{\lambda \rho} f_{, \lambda}^{\prime} \delta g_{\rho \mu}\right]+\sqrt{-g} f_{, \lambda \nu}^{\prime} \delta g^{\nu \lambda} \\
& -\frac{\sqrt{-g}}{2} f_{, \lambda}^{\prime, \lambda} g_{\mu \nu} \delta g^{\mu \nu}-\frac{\sqrt{-g}}{2} \frac{\partial}{\partial x^{\rho}}\left[g^{\mu \nu} g^{\lambda \rho} f_{, \lambda}^{\prime} \delta g_{\mu \nu}\right] \\
= & \text { derivadas totais }+f_{; \mu \nu}^{\prime} \sqrt{-g} \delta g^{\mu \nu}-\frac{1}{2} \square f^{\prime} g_{\mu \nu} \sqrt{-g} \delta g^{\mu \nu}
\end{aligned}
$$


Para o segundo integrando, obtemos:

$$
\begin{aligned}
\sqrt{-g} g^{\mu \lambda} \delta \Gamma_{\mu \sigma}^{\sigma} f_{, \lambda}^{\prime} & =\frac{\sqrt{-g}}{2} g^{\mu \lambda} g^{\sigma \rho} \delta g_{\rho \sigma, \mu} f_{, \lambda}^{\prime} \\
& =\frac{\sqrt{-g}}{2}\left[\frac{\partial}{\partial x^{\mu}}\left(g^{\mu \lambda} g^{\sigma \rho} f_{, \lambda}^{\prime} \delta g_{\rho \sigma}\right)-g^{\mu \lambda} g^{\sigma \rho} f_{, \lambda \mu}^{\prime} \delta g_{\rho \sigma}\right] \\
& =\text { derivadas totais }+\frac{1}{2} \square f^{\prime} g_{\mu \nu} \sqrt{-g} \delta g^{\mu \nu}
\end{aligned}
$$

Portanto,

$$
\delta S=\frac{1}{16 \pi G} \int\left(-\frac{f}{2} g_{\mu \nu}+f^{\prime} R_{\mu \nu}-f_{; \mu \nu}^{\prime}+\square f^{\prime} g_{\mu \nu}\right) \sqrt{-g} \delta g^{\mu \nu} d^{4} x=0
$$

ou seja,

$$
-\frac{1}{2} g_{\mu \nu} f(R)+f^{\prime}(R) R_{\mu \nu}-\left(\nabla_{\mu} \nabla_{\nu}-g_{\mu \nu} \square\right) f^{\prime}(R)=0
$$




\section{Apêndice II}

Vamos calcular em detalhes as soluções das equações de movimento (6.31), (6.32) e (6.33) nas vizinhanças de $t=0$. Em torno deste ponto, escrevemos as soluções nas formas seguintes:

$$
\begin{aligned}
H & =0+H_{1} t+H_{2} t+\mathcal{O}\left(t^{3}\right) \\
\phi & =\phi_{b}+\phi_{1} t+\phi_{2} t^{2}+\phi_{3} t^{3}+\mathcal{O}\left(t^{4}\right) \\
\rho & =\rho_{b}+\rho_{1} t+\rho_{2} t^{3}+\mathcal{O}\left(t^{4}\right)
\end{aligned}
$$

Para escrevermos $f(R)$ como uma série de potências em $t$, é necessário expressar $R$ como função de $t$ :

$$
R=6 H_{1}+12 H_{2} t+12 H_{1}^{2} t^{2}+24 H_{1} H_{2} t^{3}+12 H_{2}^{2} t^{4}+\mathcal{O}\left(t^{5}\right),
$$

de tal modo que

$$
\begin{aligned}
f(R(t))=f_{b}+ & 12 f_{1} H_{2} t+\left(12 f_{1} H_{1}^{2}+144 f_{2} H_{2}^{2}\right) t^{2}+ \\
& +\left(24 f_{1} H_{1} H_{2}+1728 f_{3} H_{2}^{3}+288 f_{2} H_{2} H_{1}^{2}\right) t^{3}+\mathcal{O}\left(t^{4}\right) .
\end{aligned}
$$

Além disso,

$$
f^{\prime}(R)=f_{1}+24 f_{2} H_{2} t+\left(24 f_{2} H_{1}^{2}+432 f_{3} H_{2}^{2}\right) t^{2}+\mathcal{O}\left(t^{3}\right)
$$

A igualdade $f^{\prime}=\phi$ implica, em ordem zero:

$$
f_{1}=\phi_{b}
$$

Em primeira ordem:

$$
f_{2}=\frac{\phi_{1}}{24 H_{2}}
$$


E, em segunda ordem:

$$
f_{3}=-\frac{1}{432} \frac{\phi_{1} H_{1}^{2}-H_{2} \phi_{2}}{H_{2}^{3}} .
$$

Precisamos também do potencial $W_{, \phi}=\left(2 f-R f^{\prime}\right) / 3$ escrito em potências de t. Usando (7.7) e (7.8), obtemos

$$
\begin{aligned}
W_{, \phi}= & \frac{2}{3} f_{b}-2 H_{1} \phi_{b}+\left(8 f_{1} H_{2}-2 H_{1} \phi_{1}-4 H_{2} \phi_{b}\right) t+ \\
& +\left(-2 H_{1} \phi_{2}-4 H_{2} \phi_{1}-4 H_{1}^{2} \phi_{b}+8 f_{1} H_{1}^{2}+96 f_{2} H_{2}^{2}\right) t^{2}+\mathcal{O}\left(t^{3}\right) .
\end{aligned}
$$

Vamos agora calcular os coeficientes da expansão (7.5) usando a equação (6.33). Em ordem zero, obtemos:

$$
\rho_{1}=0
$$

Em primeira ordem:

$$
\rho_{2}=-\frac{3}{2} H_{1}(1+w) \rho_{b}
$$

Finalmente, em ordem 2:

$$
\rho_{3}=-H_{2} \rho_{b}(1+w)
$$

Vamos passar agora à equação de movimento 0 - 0 (6.31) e à equação do traço (6.32). Em ordem 0, a equação (6.31) dá:

$$
H_{1}=\frac{f_{b}-16 \mathcal{P}_{b}}{6 \phi_{b}}
$$

onde $\mathcal{P}_{b} \equiv \pi G \rho_{b}$. A equação (6.32), por sua vez, dá, sempre em ordem 0 :

$$
\phi_{2}=-\frac{f_{b}}{6}-4 \mathcal{P}_{b}\left(\frac{1}{3}+w\right) .
$$

Em ordem 1, a equação 0 - 0 não adiciona nenhuma informação, enquanto que a equação (6.32) dá:

$$
H_{2}=\frac{1}{24 \phi_{b}^{2}}\left(-36 \phi_{3} \phi_{b}-\phi_{1} f_{b}+16 \mathcal{P}_{b} \phi_{1}\right)
$$


Em ordem 2, a equação 0 - 0 não adiciona nenhuma informação, ao passo que a equação (6.32) dá:

$$
\phi_{3}=-\frac{f b-16 \mathcal{P}_{b}}{36 \phi_{1} \phi_{b}}\left(16 \phi_{b} \mathcal{P}_{b}+32 \phi_{b} \mathcal{P}_{b} w+\phi_{1}^{2}+16 \phi_{b} \mathcal{P}_{b} w^{2}\right)
$$

Substituindo a solução (7.19) em (7.18), obtemos

$$
H_{2}=\frac{2}{3} \frac{\mathcal{P}_{b}(1+w)^{2}\left(f b-16 \mathcal{P}_{b}\right)}{\phi_{1} \rho_{b}}
$$

Por fim, a equação (6.32) em ordem 3 dá duas soluções para $\phi_{1}$, que são:

$$
\phi_{1}=-4 \varepsilon(1+w) \sqrt{\mathcal{P}_{b} \phi_{b} \gamma_{b}(w)}
$$

onde $\varepsilon= \pm 1 \mathrm{e}$

$$
\gamma_{b}(w) \equiv \frac{2\left[f_{b}+4 \mathcal{P}_{b}(3 w+7)(3 w+2)\right]}{f_{b}-16 \mathcal{P}_{b}}
$$

O sistema está assim resolvido, bastando para isso substituir a solução para $\phi_{1}$ acima nas soluções para os outros coeficientes. São eles:

$$
\begin{aligned}
H_{2} & =\varepsilon(1+w) \frac{f_{b}-16 \mathcal{P}_{b}}{6 \phi_{b}} \sqrt{\frac{\mathcal{P}_{b}}{\phi_{b} \gamma_{b}(w)}} \\
\phi_{3} & =\varepsilon \frac{(1+w)}{9 \gamma_{b}(w)} \sqrt{\frac{\mathcal{P}_{b}}{\phi_{b}}}\left(f_{b}-16 \mathcal{P}_{b}\right) \\
f_{2} & =\frac{\phi_{b}^{2} \gamma_{b}(w)}{f_{b}-16 \mathcal{P}_{b}} \\
f_{3} & =-\frac{\phi_{b}^{3} \gamma_{b}(w) \delta_{b}(w)}{24 \mathcal{P}_{b}(1+w)^{2}\left(f_{b}-16 \mathcal{P}_{b}\right)} \\
\rho_{2} & =-(1+w) \frac{f_{b}-16 \mathcal{P}_{b}}{4 \phi_{b}} \\
\rho_{3} & =-\varepsilon \frac{(1+w)^{2}}{\gamma_{b}^{1 / 2}(w)} \frac{f_{b}-16 \mathcal{P}_{b}}{6 \phi_{b}} \sqrt{\frac{\mathcal{P}_{b}}{\phi_{b}}}
\end{aligned}
$$

onde

$$
\delta_{b}(w) \equiv \frac{3 f_{b}+8 \mathcal{P}_{b}\left(12 w^{2}+37 w+19\right)}{f_{b}-16 \mathcal{P}_{b}}
$$




\section{Apêndice III}

Vamos deduzir as equações de movimento para a seguinte ação gravitacional:

$$
S=\frac{1}{16 \pi G} \int\left[R+\varphi(x) f\left(R, R_{\mu \nu} R^{\mu \nu}\right)-V(\varphi)\right] \sqrt{-g} d^{4} x
$$

onde $\varphi(x)$ é um campo escalar que depende das coordenadas do espaço-tempo. Assim como no caso de teorias $f(R)$, é útil simplificarmos o integrando calculando sua variação explicitamente em um referencial localmente inercial antes de passarmos a um referencial arbitrário para efetuarmos a integração por partes. Este último passo consiste em trocar derivadas parciais por derivadas covariantes. Também é útil lembrar da identidade (7.2) que relaciona divergentes covariantes com derivadas parciais.

A variação do primeiro termo de (7.28), $\delta(R \sqrt{-g}) / \delta g^{\mu \nu}$, é conhecida. O resultado é justamente o lado esquerdo das equações de Einstein:

$$
\frac{\delta(\sqrt{-g} R)}{\delta g^{\mu \nu}}=R_{\mu \nu}-\frac{1}{2} g_{\mu \nu} R
$$

Vamos escrever o segundo e terceiro termos nas seguintes formas:

$$
\begin{gathered}
\delta(\varphi f \sqrt{-g})=\varphi \delta(f \sqrt{-g})=\varphi\left[\sqrt{-g}\left(f_{R} \delta R+f_{P} \delta\left(R_{\mu \nu} R^{\mu \nu}\right)\right)+\delta \sqrt{-g} f\right] \\
\delta(V \sqrt{-g})=V \delta \sqrt{-g}=-\frac{V}{2} g_{\mu \nu} \delta g^{\mu \nu} \sqrt{-g}
\end{gathered}
$$

onde definimos $f_{R} \equiv \partial f / \partial R$ e $f_{P} \equiv \partial f / \partial\left(R_{\mu \nu} R^{\mu \nu}\right)$. É útil lembrar que

$$
\delta \sqrt{-g}=-\frac{g_{\mu \nu}}{2} \delta g^{\mu \nu} \sqrt{-g},
$$

termo este que aparece em (7.30).

Devemos agora reduzir a equação (7.30) em termos do fator comum $\sqrt{-g} \delta g^{\mu \nu}$. 
Vamos começar pelo primeiro termo:

$$
\begin{aligned}
\varphi f_{R} \delta R \sqrt{-g} & =\varphi f_{R}\left(R_{\mu \nu} \delta g^{\mu \nu}+g^{\mu \nu} \delta R_{\mu \nu}\right) \sqrt{-g} \\
& =\varphi f_{R}\left[R_{\mu \nu} \delta g^{\mu \nu}+\frac{\partial}{\partial x^{\lambda}}\left(g^{\mu \nu} \delta \Gamma_{\mu \nu}^{\lambda}-g^{\mu \lambda} \delta \Gamma_{\mu \nu}^{\nu}\right)\right] \sqrt{-g} \\
& =\varphi f_{R}\left[R_{\mu \nu} \delta g^{\mu \nu} \sqrt{-g}+\frac{\partial}{\partial x^{\lambda}}\left(\sqrt{-g}\left(g^{\mu \nu} \delta \Gamma_{\mu \nu}^{\lambda}-g^{\mu \lambda} \delta \Gamma_{\mu \nu}^{\nu}\right)\right)\right]
\end{aligned}
$$

O primeiro termo da equação (??) já está na forma desejada. Podemos agora fazer uma integração por partes do segundo termo, ou seja,

$$
\begin{aligned}
\int \varphi f_{R} \frac{\partial}{\partial x^{\lambda}}\left(\sqrt { - g } \left(g^{\mu \nu}\right.\right. & \left.\left.\delta \Gamma_{\mu \nu}^{\lambda}-g^{\mu \lambda} \delta \Gamma_{\mu \nu}^{\nu}\right)\right)= \\
& =-\int\left(\varphi f_{R}\right)_{, \lambda}\left(g^{\mu \nu} \delta \Gamma_{\mu \nu}^{\lambda}-g^{\mu \lambda} \delta \Gamma_{\mu \nu}^{\nu}\right) \sqrt{-g} d^{4} x .
\end{aligned}
$$

O primeiro integrando em (7.33) pode ser simplificado agrupando os termos como derivadas totais. Explicitamente,

$$
\begin{gathered}
\left(\varphi f_{R}\right)_{, \lambda} g^{\mu \nu} \delta \Gamma_{\mu \nu}^{\lambda} \sqrt{-g}=\left(\varphi f_{R}\right)_{, \lambda} \frac{g^{\mu \nu} g^{\lambda \rho}}{2}\left[\delta g_{\rho \mu, \nu}+\delta g_{\rho \nu, \mu}-\delta g_{\mu \nu, \rho}\right] \sqrt{-g}= \\
=\frac{\partial}{\partial x^{\nu}}\left[\left(\varphi f_{R}\right)_{, \lambda} \frac{g^{\mu \nu} g^{\lambda \rho}}{2} \delta g_{\rho \mu}\right] \sqrt{-g}-\left(\varphi f_{R}\right)_{, \lambda} \frac{\partial}{\partial x^{\nu}}\left[\left(\varphi f_{R}\right)_{, \lambda} \frac{g^{\mu \nu} g^{\lambda \rho}}{2}\right] \sqrt{-g} \delta g_{\rho \mu} \\
+\frac{\partial}{\partial x^{\mu}}\left[\left(\varphi f_{R}\right)_{, \lambda} \frac{g^{\mu \nu} g^{\lambda \rho}}{2} \delta g_{\rho \nu}\right] \sqrt{-g}-\frac{\partial}{\partial x^{\mu}}\left[\left(\varphi f_{R}\right)_{, \lambda} \frac{g^{\mu \nu} g^{\lambda \rho}}{2}\right] \sqrt{-g} \delta g_{\rho \nu} \\
-\frac{\partial}{\partial x^{\rho}}\left[\left(\varphi f_{R}\right)_{, \lambda} \frac{g^{\mu \nu} g^{\lambda \rho}}{2} \delta g_{\mu \nu}\right] \sqrt{-g}+\frac{\partial}{\partial x^{\rho}}\left[\left(\varphi f_{R}\right)_{, \lambda} \frac{g^{\mu \nu} g^{\lambda \rho}}{2}\right] \sqrt{-g} \delta g_{\mu \nu}= \\
=\frac{\partial}{\partial x^{\nu}}\left[\left(\varphi f_{R}\right)_{, \lambda} g^{\mu \nu} g^{\lambda \rho} \delta g_{\rho \mu} \sqrt{-g}\right]-\nabla_{\mu}\left[\left(\varphi f_{R}\right)_{, \lambda}\right] g^{\mu \nu} g^{\lambda \rho} \delta g_{\rho \nu} \sqrt{-g} \\
-\frac{\partial}{\partial x^{\rho}}\left[\left(\varphi f_{R}\right)_{, \lambda} \frac{g^{\mu \nu} g^{\lambda \rho}}{2} \delta g_{\mu \nu} \sqrt{-g}\right]+\nabla_{\rho}\left[\frac{\left(\varphi f_{R}\right)_{, \lambda}}{2}\right] g^{\mu \nu} g^{\lambda \rho} \delta g_{\mu \nu} \sqrt{-g}
\end{gathered}
$$

É claro que os termos de derivada total (primeiro e terceiro termos da expressão acima) em nada contribuem para as equações de movimento. Simplificamos o segundo integrando de (7.33) de modo similar ao primeiro. O resultado é:

$$
\begin{aligned}
\left(\varphi f_{R}\right)_{, \lambda} g^{\mu \lambda} \delta \Gamma_{\mu \nu}^{\nu} \sqrt{-g}=\frac{\partial}{\partial x^{\rho}}\left[\sqrt{-g}\left(\varphi f_{R}\right)_{, \lambda} \frac{g^{\rho \lambda} g^{\nu \mu}}{2} \delta g_{\mu \nu}\right] \\
-\nabla_{\rho}\left[\frac{\left(\varphi f_{R}\right)_{, \lambda}}{2}\right] g^{\rho \lambda} g^{\nu \mu} \delta g_{\mu \nu} \sqrt{-g}
\end{aligned}
$$


Coletando os resultados (7.34) e (7.35), chegamos à seguinte expressão para (7.33):

$$
\begin{array}{r}
-\int\left(\varphi f_{R}\right)_{, \lambda}\left(g^{\mu \nu} \delta \Gamma_{\mu \nu}^{\lambda}-g^{\mu \lambda} \delta \Gamma_{\mu \nu}^{\nu}\right) \sqrt{-g} d^{4} x=-\int\left(\varphi f_{R}\right)_{; \nu ; \mu} \delta g^{\nu \mu} \sqrt{-g} d^{4} x \\
+\int\left(\varphi f_{R}\right)_{; \lambda}^{; \lambda} g_{\mu \nu} \delta g^{\mu \nu} \sqrt{-g} d^{4} x
\end{array}
$$

Passemos ao segundo termo de (7.30):

$$
\varphi f_{P} \delta\left(R_{\mu \nu} R^{\mu \nu}\right) \sqrt{-g}=2 \varphi f_{P} \sqrt{-g}\left(\delta R_{\mu \nu} g^{\mu \rho} R_{\rho}^{v}+R_{\mu \nu} R_{\rho}^{\nu} \delta g^{\mu \rho}\right)
$$

O segundo termo de (7.37) já está na forma desejada. Vamos então desenvolver o primeiro termo. De modo completamente análogo ao cálculo feito em (7.34) temos:

$$
\begin{gathered}
\varphi f_{P} \sqrt{-g} \delta R_{\mu \nu} g^{\mu \rho} R_{\rho}^{\nu}=\frac{\partial}{\partial x^{\lambda}}\left[\sqrt{-g} \varphi f_{P} g^{\mu \rho} \delta \Gamma_{\mu \nu}^{\lambda} R_{\rho}^{\nu}\right]-\frac{\partial}{\partial x^{\lambda}}\left[\sqrt{-g} \varphi f_{P} g^{\mu \rho} \delta \Gamma_{\mu \kappa}^{\kappa} R_{\rho}^{\lambda}\right] \\
-\sqrt{-g} \nabla_{\lambda}\left[\varphi f_{P} g^{\mu \rho} R_{\rho}^{\nu}\right] \frac{g^{\lambda \sigma}}{2}\left(\delta g_{\sigma \mu ; \nu}-\delta g_{\sigma \nu ; \mu}-\delta g_{\mu \nu ; \sigma}\right) \\
+\sqrt{-g} \nabla_{\lambda}\left[\varphi f_{P} g^{\mu \rho} R_{\rho}^{\lambda}\right] \frac{g^{\kappa \sigma}}{2} \delta g_{\sigma \kappa ; \mu}
\end{gathered}
$$

É claro que os termos de derivada total (primeiro e segundo termos de (7.38)) em nada contribuem para as equações de campo. Devemos agora desenvolver os termos proporcionais às derivadas covariantes da métrica como segue:

$$
\begin{aligned}
& \sqrt{-g} \nabla_{\lambda}\left[\varphi f_{P} g^{\mu \rho} R_{\rho}^{\nu}\right] \frac{g^{\lambda \sigma}}{2} \delta g_{\sigma \mu ; \nu}=\sqrt{-g} \frac{\partial}{\partial x^{\lambda}}\left[\varphi f_{P} g^{\mu \rho} R_{\rho}^{\nu}\right] \frac{g^{\lambda \sigma}}{2} \delta g_{\sigma \mu, \nu}= \\
& =\frac{\partial}{\partial x^{\nu}}\left[\sqrt{-g}\left(\varphi f_{P} R^{\mu \nu}\right)_{, \lambda} \frac{g^{\lambda \sigma}}{2} \delta g_{\sigma \mu}\right]-\sqrt{-g} \nabla_{\nu}\left[\left(\varphi f_{P} R^{\mu \nu}\right)_{; \lambda}\right] \frac{g^{\lambda \sigma}}{2} \delta g_{\sigma \mu}, \\
& \sqrt{-g} \nabla_{\lambda}\left[\varphi f_{P} R^{\mu \nu}\right] \frac{g^{\lambda \sigma}}{2} \delta g_{\sigma \nu ; \mu}=\sqrt{-g} \frac{\partial}{\partial x^{\lambda}}\left[\varphi f_{P} R^{\mu \nu}\right] \frac{g^{\lambda \sigma}}{2} \delta g_{\sigma \nu, \mu}= \\
& =\frac{\partial}{\partial x^{\mu}}\left[\sqrt{-g}\left(\varphi f_{P} R^{\mu \nu}\right)_{, \lambda} \frac{g^{\lambda \sigma}}{2} \delta g_{\sigma \nu}\right]-\sqrt{-g} \nabla_{\mu}\left[\left(\varphi f_{P} R^{\mu \nu}\right)_{; \lambda}\right] \frac{g^{\lambda \sigma}}{2} \delta g_{\sigma \nu},
\end{aligned}
$$




$$
\begin{aligned}
\sqrt{-g} \nabla_{\lambda}\left[\varphi f_{P} R^{\mu \nu}\right] \frac{g^{\lambda \sigma}}{2} \delta g_{\mu \nu ; \sigma}=\frac{\partial}{\partial x^{\sigma}} & {\left[\sqrt{-g}\left(\varphi f_{P} R^{\mu \nu}\right)_{, \lambda} \frac{g^{\lambda \sigma}}{2} \delta g_{\mu \nu}\right] } \\
& -\sqrt{-g} \nabla_{\sigma}\left[\left(\varphi f_{P} R^{\mu \nu}\right)_{; \lambda}\right] \frac{g^{\lambda \sigma}}{2} \delta g_{\mu \nu} \\
\sqrt{-g} \nabla_{\lambda}\left[\varphi f_{P} R^{\mu \lambda}\right] \frac{g^{\kappa \sigma}}{2} \delta g_{\sigma \kappa ; \mu}=\frac{\partial}{\partial x^{\mu}} & {\left[\sqrt{-g}\left(\varphi f_{P} R^{\mu \lambda}\right)_{, \lambda} \frac{g^{\kappa \sigma}}{2} \delta g_{\sigma \kappa}\right] } \\
& -\sqrt{-g} \nabla_{\mu}\left[\left(\varphi f_{P} R^{\mu \lambda}\right)_{; \lambda}\right] \frac{g^{\kappa \sigma}}{2} \delta g_{\sigma \kappa}
\end{aligned}
$$

Logo, a integral da expressão (7.38) fica

$$
\begin{aligned}
\int \varphi f_{P} \delta R_{\mu \nu} R^{\mu \nu} \sqrt{-g} & d^{4} x=-\frac{1}{2} \int\left[\left(\varphi f_{P} R_{\nu}^{\lambda}\right)_{; \mu ; \lambda}+\left(\varphi f_{P} R_{\mu \nu}\right)_{; \lambda}^{; \lambda}\right. \\
& \left.+\left(\varphi f_{P} R^{\rho \lambda}\right)_{; \lambda ; \rho} g_{\mu \nu}-\left(\varphi f_{P} R_{\mu}^{\lambda}\right)_{; \nu \lambda}\right] \delta g^{\mu \nu} \sqrt{-g} d^{4} x
\end{aligned}
$$

Finalmente,

$$
\begin{aligned}
\delta S=\frac{1}{16 \pi G} \int & {\left[R_{\mu \nu}-\frac{1}{2} g_{\mu \nu} R+\varphi f_{R} R_{\mu \nu}-\left(\varphi f_{R}\right)_{; \nu \mu}+\left(\varphi f_{R}\right)_{; \lambda}^{; \lambda} g_{\mu \nu}\right.} \\
& +2 R_{\mu \lambda} R_{\nu}^{\lambda} \varphi f_{P}+\left(\varphi f_{P} R_{\mu \nu}\right)_{; \lambda}^{; \lambda}+\left(\varphi f_{P} R^{\rho \lambda}\right)_{; \lambda \rho} g_{\mu \nu} \\
& \left.-2\left(\varphi f_{P} R_{(\mu}^{\lambda} \delta_{\nu)}^{\rho}\right)_{; \rho \lambda}-\frac{f}{2} g_{\mu \nu}-\frac{V(\varphi)}{2} g_{\mu \nu}\right] \delta g^{\mu \nu} \sqrt{-g} d^{4} x .
\end{aligned}
$$

Para calcular as componentes das equações (7.44) para uma métrica arbitrária, programas algébricos tais como Maple e Mathematica, com pacotes externos GRTensorII (Maple) e xAct (Mathematica), podem ser úteis. Para o caso particular do espaço-tempo de Friedmann-Robertson-Walker, é muito mais simples substituir a métrica diretamente na ação e efetuar a variação com respeito ao fator de escala, o que resultará na componente espacial das equações (7.44), e, para se obter a componente $t-t$, efetuar a variação com respeito à função lapso $N(t)$. No final dos cálculos, basta fazer a escolha $N(t)=1$. A forma geral (7.44) também pode ser útil para se efetuar um cálculo perturbativo, ou seja, podemos perturbar diretamente as equações (7.44) em vez de expandir a ação original até segunda ordem. 


\section{Referências Bibliográficas}

[1] E. Komatsu et al., (2010), 1001.4538.

[2] A. H. Guth, Phys. Rev. D 23, 347 (1981).

[3] A. D. Linde, Phys. Lett. B108, 389 (1982).

[4] A. Albrecht and P. J. Steinhardt, Phys. Rev. Lett. 48, 1220 (1982).

[5] A. Borde and A. Vilenkin, Phys. Rev. D 56, 717 (1997).

[6] A. Borde and A. Vilenkin, Phys. Rev. Lett. 72, 3305 (1994).

[7] A. Borde, A. H. Guth, and A. Vilenkin, Phys. Rev. Lett. 90, 151301 (2003).

[8] G. L. Murphy, Phys. Rev. D 8, 4231 (1973).

[9] V. N. Melnikov and S. V. Orlov, Physics Letters A 70, 263 (1979).

[10] J. C. Fabris, R. G. Furtado, P. Peter, and N. Pinto-Neto, Phys. Rev. D 67, 124003 (2003).

[11] J. Martin and P. Peter, Phys. Rev. D68, 103517 (2003), hep-th/0307077.

[12] J. Martin, P. Peter, N. Pinto Neto, and D. J. Schwarz, Phys. Rev. D65, 123513 (2002), hep-th/0112128.

[13] L. R. Abramo, I. Yasuda, and P. Peter, Phys. Rev. D81, 023511 (2010), 0910.3422.

[14] M. Gasperini and G. Veneziano, Phys. Rept. 373, 1 (2003), hepth/0207130.

[15] J. E. Lidsey, D. Wands, and E. J. Copeland, Physics Reports 337, 343 (2000). 
[16] J. Khoury, B. A. Ovrut, P. J. Steinhardt, and N. Turok, Phys. Rev. D64, 123522 (2001), hep-th/0103239.

[17] J. Khoury, B. A. Ovrut, N. Seiberg, P. J. Steinhardt, and N. Turok, Phys. Rev. D 65, 086007 (2002).

[18] P. Peter and N. Pinto-Neto, Phys. Rev. D78, 063506 (2008), 0809.2022.

[19] R. Brustein and G. Veneziano, Phys. Lett. B329, 429 (1994), hepth/9403060.

[20] C. Armendariz-Picon, V. F. Mukhanov, and P. J. Steinhardt, Phys. Rev. D63, 103510 (2001), astro-ph/0006373.

[21] L. R. Abramo and P. Peter, JCAP 0709, 001 (2007), arXiv:0705.2893 [astro-ph].

[22] L. R. Abramo and N. Pinto-Neto, Phys. Rev. D73, 063522 (2006), astro$\mathrm{ph} / 0511562$.

[23] V. F. Mukhanov and R. H. Brandenberger, Phys. Rev. Lett. 68, 1969 (1992).

[24] R. Brandenberger, V. Mukhanov, and A. Sornborger, Phys. Rev. D 48, 1629 (1993).

[25] R. H. Brandenberger, R. Easther, and J. Maia, JHEP 08, 007 (1998), gr-qc/9806111.

[26] G. 't Hooft and M. J. G. Veltman, Annales Inst. H. Poincare A20, 69 (1974).

[27] S. Deser, H.-S. Tsao, and P. van Nieuwenhuizen, Phys. Rev. D 10, 3337 (1974).

[28] K. S. Stelle, Phys. Rev. D 16, 953 (1977).

[29] T. Chiba, JCAP 0503, 008 (2005), gr-qc/0502070.

[30] A. Nunez and S. Solganik, Phys. Lett. B608, 189 (2005), hepth/0411102.

[31] R. P. Woodard, Lect. Notes Phys. 720, 403 (2007), astro-ph/0601672. 
[32] J.-P. Bruneton and G. Esposito-Farese, Phys. Rev. D76, 124012 (2007), 0705.4043.

[33] S. Weinberg, Gravitation and Cosmology, John Wiley .

[34] A. S. Eddington, The Mathematical Theory of Relativity, 2nd ed. (Cambridge University Press, London), 162 (1924).

[35] C. Wirtz, Astr. Nachr. 206, 109 (1918).

[36] C. Wirtz, Astr. Nachr. 215, 349 (1921).

[37] C. Wirtz, Astr. Nachr. 216, 451 (1922).

[38] C. Wirtz, Astr. Nachr. 222, 21 (1924).

[39] C. Wirtz, Scientia 38, 303 (1925).

[40] C. Lundmark, K., Stock. Hand. 50, No. 8 (1920).

[41] C. Lundmark, K., Mon. Not. Roy. Astron. Soc. 84, 747 (1924).

[42] E. Hubble, Proc. Nat. Acad. Sci. 15, 168 (1929).

[43] W. L. Freedman et al., The Astrophysical Journal 553, 47 (2001).

[44] G. Gamow, Phys. Rev. 74, 505 (1948).

[45] R. A. Alpher and R. C. Herman, Phys. Rev. 75, 1089 (1949).

[46] D. J. Fixsen et al., Astrophys. J. 473, 576 (1996), astro-ph/9605054.

[47] Y. B. Zel'dovich, Adv. Astron. Astrophys. 3, 241 (1965).

[48] P. J. E. Peebles, Astrophys. J. 146, 542 (1966).

[49] T. P. Walker, G. Steigman, D. N. Schramm, K. A. Olive, and H.-S. Kang, Astrophys. J. 376, 51 (1991).

[50] G. Steigman, (2003), astro-ph/0307244.

[51] P. J. E. Peebles, D. N. Schramm, E. L. Turner, and R. G. Kron, Nature 352, 769 (1991). 
[52] N. A. Bahcall, J. P. Ostriker, S. Perlmutter, and P. J. Steinhardt, Science 284, 1481 (1999), astro-ph/9906463.

[53] Supernova Cosmology Project, S. Perlmutter et al., Astrophys. J. 517, 565 (1999), astro-ph/9812133.

[54] S. Weinberg, Rev. Mod. Phys. 61, 1 (1989).

[55] J. D. Barrow and A. C. Ottewill, J. Phys. A16, 2757 (1983).

[56] W. Hu and I. Sawicki, Phys. Rev. D76, 064004 (2007), 0705.1158.

[57] A. A. Starobinsky, JETP Lett. 86, 157 (2007), 0706.2041.

[58] S. Tsujikawa, Phys. Rev. D77, 023507 (2008), 0709.1391.

[59] V. Miranda, S. E. Joras, I. Waga, and M. Quartin, Phys. Rev. Lett. 102, 221101 (2009), 0905.1941.

[60] I. Thongkool, M. Sami, R. Gannouji, and S. Jhingan, Phys. Rev. D 80, 043523 (2009).

[61] A. de la Cruz-Dombriz, A. Dobado, and A. L. Maroto, Phys. Rev. Lett. 103, 179001 (2009).

[62] A. V. Frolov, Phys. Rev. Lett. 101, 061103 (2008), 0803.2500.

[63] B. A. Bassett, S. Tsujikawa, and D. Wands, Rev. Mod. Phys. 78, 537 (2006), astro-ph/0507632.

[64] A. Linde, Parcticle Physics and Inflationary Cosmology, Harwood (1990).

[65] V. F. Mukhanov, H. A. Feldman, and R. H. Brandenberger, Phys. Rept. 215, 203 (1992).

[66] J. M. Bardeen, Phys. Rev. D22, 1882 (1980).

[67] F. Finelli and R. Brandenberger, Phys. Rev. Lett. 82, 1362 (1999).

[68] S. Weinberg, Phys. Rev. D67, 123504 (2003), astro-ph/0302326.

[69] E. F. Bunn, A. R. Liddle, and M. White, Phys. Rev. D 54, R5917 (1996). 
[70] D. H. Lyth and A. Riotto, Phys. Rept. 314, 1 (1999), hep-ph/9807278.

[71] J. Martin and R. H. Brandenberger, Phys. Rev. D 63, 123501 (2001).

[72] M. Novello and S. E. P. Bergliaffa, Phys. Rept. 463, 127 (2008), 0802.1634 .

[73] R. C. Tolman, Phys. Rev. 38, 1758 (1931).

[74] G. Lemaitre, Ann. Soc. Sci. Bruxelles (1933).

[75] S. Hawking and G. Ellis, The large scale structure of space-time, Cambridge .

[76] V. A. Belinski and I. M. Khalatnikov, Soviet Physics-JETP 29, 911 (1969).

[77] V. A. Belinski, E. M. Lifshitz, and I. M. Khalatnikov, Advances in Physics 19, 525 (1970).

[78] R. M. Wald, General Relativity, Chicago, Usa: Univ. Pr. ( 1984) 491p .

[79] E. Poisson, A Relativist's toolkit, Cambridge University Press, 2004.

[80] S. Kar and S. SenGupta, Pramana 69, 49 (2007), gr-qc/0611123.

[81] L. H. Ford, Int. J. Theor. Phys. 42, 1219 (2003), gr-qc/0301045.

[82] G. F. R. Ellis and H. van Elst, NATO Adv. Study Inst. Ser. C. Math. Phys. Sci. 541, 1 (1999), gr-qc/9812046.

[83] A. A. Starobinsky, Sov. Astron. Lett. 4, 82 (1978).

[84] R. Durrer and J. Laukenmann, Classical and Quantum Gravity 13, 1069 (1996).

[85] G. Veneziano, (1997), hep-th/9802057.

[86] P. Peter, E. J. C. Pinho, and N. Pinto-Neto, Phys. Rev. D75, 023516 (2007), hep-th/0610205.

[87] R. Lehoucq, J. Weeks, J.-P. Uzan, E. Gausmann, and J.-P. Luminet, Class. Quant. Grav. 19, 4683 (2002), gr-qc/0205009. 
[88] P. Peter and J. P. Uzan, Primordial Cosmology, Oxford University Press, 2009 .

[89] J. M. Stewart, Class. Quant. Grav. 7, 1169 (1990).

[90] E. J. C. Pinho and N. Pinto-Neto, Phys. Rev. D76, 023506 (2007), hep-th/0610192.

[91] P. R. Holland, Phys. Rept. 224, 95 (1993).

[92] N. Pinto-Neto, Phys. Rev. D79, 083514 (2009), 0904.4454.

[93] M. Gasperini, M. Maggiore, and G. Veneziano, Nucl. Phys. B494, 315 (1997), hep-th/9611039.

[94] R. Brustein and R. Madden, Phys. Rev. D57, 712 (1998), hepth/9708046.

[95] C. Cartier, J.-c. Hwang, and E. J. Copeland, Phys. Rev. D64, 103504 (2001), astro-ph/0106197.

[96] R. Durrer and F. Vernizzi, Phys. Rev. D 66, 083503 (2002).

[97] I. P. C. Heard and D. Wands, Class. Quant. Grav. 19, 5435 (2002), gr-qc/0206085.

[98] D. Wands, Phys. Rev. D 60, 023507 (1999).

[99] D. H. Lyth, Phys. Lett. B524, 1 (2002), hep-ph/0106153.

[100] R. Brandenberger and F. Finelli, JHEP 11, 056 (2001), hep-th/0109004.

[101] J.-c. Hwang, Phys. Rev. D65, 063514 (2002), astro-ph/0109045.

[102] D. H. Lyth, Phys. Lett. B526, 173 (2002), hep-ph/0110007.

[103] S. Tsujikawa, R. Brandenberger, and F. Finelli, Phys. Rev. D66, 083513 (2002), hep-th/0207228.

[104] S. Tsujikawa, Phys. Lett. B526, 179 (2002), gr-qc/0110124.

[105] F. Finelli and R. Brandenberger, Phys. Rev. D65, 103522 (2002), hepth/0112249. 
[106] W. H. Kinney, E. W. Kolb, A. Melchiorri, and A. Riotto, Phys. Rev. D 74, $023502(2006)$.

[107] F. T. Falciano, M. Lilley, and P. Peter, Phys. Rev. D77, 083513 (2008), 0802.1196 .

[108] V. Bozza and G. Veneziano, Phys. Lett. B625, 177 (2005), hepth/0502047.

[109] F. Finelli, P. Peter, and N. Pinto-Neto, Phys. Rev. D77, 103508 (2008), 0709.3074.

[110] C. Boehm, P. Fayet, and J. Silk, Phys. Rev. D69, 101302 (2004), hep$\mathrm{ph} / 0311143$.

[111] E. Hayashi et al., Mon. Not. Roy. Astron. Soc. 355, 794 (2004), astro$\mathrm{ph} / 0310576$.

[112] B. Ratra and P. J. E. Peebles, Phys. Rev. D37, 3406 (1988).

[113] R. R. Caldwell, R. Dave, and P. J. Steinhardt, Astrophys. Space Sci. 261, 303 (1998).

[114] I. Zlatev, L.-M. Wang, and P. J. Steinhardt, Phys. Rev. Lett. 82, 896 (1999), astro-ph/9807002.

[115] M. Milgrom, Astrophys. J. 270, 365 (1983).

[116] R. H. Sanders and S. S. McGaugh, Ann. Rev. Astron. Astrophys. 40, 263 (2002), astro-ph/0204521.

[117] R. B. Tully and J. R. Fisher, Astron. Astrophys. 54, 661 (1977).

[118] A. De Felice and S. Tsujikawa, (2010), 1002.4928.

[119] I. Navarro and K. Van Acoleyen, JCAP 0603, 008 (2006), gr-qc/0511045.

[120] G. Cognola, E. Elizalde, S. Nojiri, S. D. Odintsov, and S. Zerbini, Phys. Rev. D73, 084007 (2006), hep-th/0601008.

[121] A. De Felice, M. Hindmarsh, and M. Trodden, JCAP 0608, 005 (2006), astro-ph/0604154. 
[122] M. Ostrogradski, Ac. St. Petersbourg Rev. VI 4, 385 (1856).

[123] D. A. Eliezer and R. P. Woodard, Nucl. Phys. B325, 389 (1989).

[124] P. Teyssandier and P. Tourrenc, J. Math. Phys. 24, 2793 (1983).

[125] C. Brans and R. H. Dicke, Phys. Rev. 124, 925 (1961).

[126] P. Jordan, Z. Phys. 157, 112 (1959).

[127] M. Fierz, Helv. Phys. Acta 29, 128 (1956).

[128] B. Bertotti, L. Iess, and P. Tortora, Nature 425, 374 (2003).

[129] Y. Fujii and K. Maeda, The Scalar-Tensor Theory of Gravitation, Cambridge USA (2003) .

[130] G. J. Olmo, Phys. Rev. D72, 083505 (2005).

[131] J. Khoury and A. Weltman, Phys. Rev. Lett. 93, 171104 (2004), astro$\mathrm{ph} / 0309300$.

[132] J. Khoury and A. Weltman, Phys. Rev. D69, 044026 (2004), astro$\mathrm{ph} / 0309411$.

[133] D. F. Mota and J. D. Barrow, Mon. Not. Roy. Astron. Soc. 349, 291 (2004), astro-ph/0309273.

[134] D. F. Mota and J. D. Barrow, Phys. Lett. B581, 141 (2004), astro$\mathrm{ph} / 0306047$.

[135] S. Tsujikawa, K. Uddin, and R. Tavakol, Phys. Rev. D77, 043007 (2008), 0712.0082 .

[136] L. Amendola, R. Gannouji, D. Polarski, and S. Tsujikawa, Phys. Rev. D75, 083504 (2007), gr-qc/0612180.

[137] R. W. Hellings et al., Phys. Rev. Lett. 51, 1609 (1983).

[138] S. E. Thorsett, Phys. Rev. Lett. 77, 1432 (1996).

[139] A. D. Dolgov and M. Kawasaki, Phys. Lett. B573, 1 (2003), astro$\mathrm{ph} / 0307285$. 
[140] I. Sawicki and W. Hu, Phys. Rev. D75, 127502 (2007), astro$\mathrm{ph} / 0702278$.

[141] S. M. Carroll, V. Duvvuri, M. Trodden, and M. S. Turner, Phys. Rev. D 70, 043528 (2004).

[142] J.-c. Hwang and H.-r. Noh, Phys. Rev. D65, 023512 (2002), astro$\mathrm{ph} / 0102005$.

[143] H. Kodama and M. Sasaki, Progress of Theoretical Physics Supplement 78, 1 (1984).

[144] J.-c. Hwang, Astrophys. J. 375, 443 (1991).

[145] B. Boisseau, G. Esposito-Farese, D. Polarski, and A. A. Starobinsky, Phys. Rev. Lett. 85, 2236 (2000), gr-qc/0001066.

[146] S. Appleby, R. Battye, and A. Starobinsky, (2009), 0909.1737.

[147] C. Lanczos, Annals Math. 39, 842 (1938).

[148] A. Salam and J. A. Strathdee, Phys. Rev. D18, 4480 (1978).

[149] H. W. Hamber, Quantum gravitation: The Feynman path integral approach, Berlin, Germany, Springer (2009) .

[150] C. Kiefer, Quantum Gravity, London, UK, Oxford University Press (2007).

[151] K. S. Stelle, Gen. Relat. Grav. 9, 353 (1978).

[152] M. Markov, JETP Lett. 36, 265 (1982).

[153] B. Altshuler, Class. Quantum Grav. 7, 189 (1990).

[154] R. Penrose, in General Relativity: An Einstein Centenary, Cambridge University Press, England, 1979.

[155] J. Martin and P. Peter, Phys. Rev. Lett. 92, 061301 (2004). 